\title{
Deep X-ray survey of the young open cluster NGC 2516 with XMM-Newton $\star, \star \star$
}

\author{
I. Pillitteri ${ }^{1}$, G. Micela ${ }^{2}$, F. Damiani ${ }^{2}$, and S. Sciortino ${ }^{2}$ \\ 1 Università degli Studi di Palermo, Piazza del Parlamento 1, 90134 Palermo, Italy \\ e-mail: pilli@astropa.unipa.it \\ 2 INAF - Osservatorio Astronomico di Palermo Piazza del Parlamento 1, 90134 Palermo, Italy \\ e-mail: [giusi;damiani;sciorti]@astropa.unipa.it
}

Received 6 August 2005 / Accepted 6 January 2006

ABSTRACT

Aims. We report a deep X-ray survey of the young ( 140 Myr), rich open cluster NGC 2516 obtained with the EPIC camera on board the XMM-Newton satellite.

Methods. By combining data from six observations, a high sensitivity, greater than a factor of 5 with respect to recent Chandra observations, has been achieved. Kaplan-Meier estimators of the cumulative X-ray luminosity distribution are built, statistically corrected for non members contaminants and compared to those of the nearly coeval Pleiades. The EPIC spectra of the X-ray brightest stars are fitted using optically thin model plasma with one or two thermal components.

Results. We detected 431 X-ray sources and 234 of them have as optical counterparts cluster stars spanning the entire NGC 2516 Main Sequence. On the basis of X-ray emission and optical photometry, we indicate 20 new candidate members of the cluster; at the same time we find $49 \mathrm{X}$-ray sources without known optical or infrared counterpart. The X-ray luminosities of cluster stars span the range log $L_{\mathrm{X}}$ $\left(\mathrm{erg} \mathrm{s}^{-1}\right)=28.4-30.8$. The representative temperatures span the $0.3-0.6 \mathrm{keV}(3.5-8 \mathrm{MK})$ range for the cool component and 1.0-2.0 keV (12-23 MK) for the hot one; similar values are found in other young open clusters like the Pleiades, IC 2391, and Blanco 1. While no significant differences are found in X-ray spectra, NGC 2516 solar type stars are definitely less luminous in X-rays than the nearly coeval Pleiades. The comparison with a previous ROSAT survey reveals the lack of variability amplitudes larger than a factor of 2 in solar type stars in a $\sim 11 \mathrm{yr}$ time scale of the cluster and thus activity cycles like in the Sun are probably absent or have a different period and amplitude in young stars.

Key words. X-ray: stars - stars: activity - open clusters and associations: individual: NGC 2516

\section{Introduction}

$\mathrm{X}$-ray observations of stars have shown that X-ray emission is a common feature along the main sequence (Vaiana et al. 1981). The emission mechanisms depend on mass: in massive $\mathrm{O}$ and early $\mathrm{B}$ type stars the $\mathrm{X}$-rays are likely generated by plasma shocked and heated in stellar winds, although a role of the magnetic field is not ruled out (Lucy \& White 1980; Feldmeier 1995; Babel \& Montmerle 1997; Cassinelli \& MacGregor 2000; Waldron \& Cassinelli 2001); solar mass stars present coronae composed of hot, magnetically confined emitting plasma, as directly observed in the Sun (Vaiana et al. 1981). Only B-late - A-early stars are not expected to emit

\footnotetext{
* Based on observations obtained with XMM-Newton, an ESA science mission with instruments and contributions directly funded by ESA Member States and NASA.

$\star \star$ Appendices A-D are only available in electronic form at http://www. edpsciences.org
}

significant X-rays because neither strong stellar winds nor convective regions, necessary to have a solar magnetic dynamo, are present in these stars. Focusing on solar mass stars, the X-ray activity is strictly linked to the internal structure and the rotation. The coupling of convective motion and rotation produces the solar-type dynamo. The loss of angular momentum is the main factor leading to a decrease of X-ray activity during stellar evolution, especially in the first Gyr, although the role of other factors like stellar chemical composition or environmental forming conditions cannot be ruled out.

In this context the studies based on stellar open clusters with different properties are of great importance to better define the relevance of age differences, chemical composition and environmental influence. An open cluster like the Pleiades constitutes a prototype for the study of activity and evolution at an age around $100 \mathrm{Myr}$, given its richness and nearby location. The evolution of X-ray emission with stellar age has been 
Table 1. Log of XMM-Newton observations of NGC 2516. All exposures have been taken with the Thick filter. Positions refer to J2000, times (referred to the pn exposures) are in kiloseconds.

\begin{tabular}{llllll}
\hline \hline OBS Id. & Orbit Nr. & RA & Dec & Time (filtered/total) & Date \\
\hline 0113891001 & 0060 & $7: 58: 20$ & $-60: 52: 13$ & $20.0 / 20.0$ & 2000-04-06 @ 19:25:05 \\
0113891101 & 0060 & $7: 58: 20$ & $-60: 52: 13$ & $14.5 / 17.0$ & 2000-04-07 @ 04:05:31 \\
0126511201 & 0092 & $7: 58: 22$ & $-60: 45: 36$ & $21.9 / 27.5$ & 2000-06-10 @ 08:56:51 \\
0134531201 & 0209 & $7: 58: 22$ & $-60: 45: 36$ & $18.8 / 19.0$ & 2001-01-29 @ 00:23:20 \\
0134531301 & 0209 & $7: 58: 22$ & $-60: 45: 36$ & $9.3 / 9.3$ & 2001-01-29 @ 08:01:48 \\
0134531501 & 0346 & $7: 58: 22$ & $-60: 45: 36$ & $21.2 / 21.2$ & 2001-10-29 @ 09:53:59 \\
\hline
\end{tabular}

studied in the last five years with XMM-Newton and Chandra $\mathrm{X}$-ray observatories, which provided high quality surveys of open clusters like Pleiades, Blanco 1, IC 2391, NGC 6383, NGC 188, M 67 (cf. Briggs \& Pye 2003; Pillitteri et al. 2004; Marino et al. 2004; Rauw et al. 2003; van den Berg et al. 2004; Gondoin 2005). In this paper we study the X-ray emission of the young open cluster NGC 2516.

NGC 2516 is a rich open cluster with an age of $140 \mathrm{Myr}$ (Meynet et al. 1993), slightly older than the Pleiades, located in the southern hemisphere at a height of $\sim 100 \mathrm{pc}$ on the Galactic Plane. The richness of star and its relatively nearby location (about $387 \mathrm{pc}$ ) have motivated the growing interest in the study of this cluster both in the optical and in the X-ray band. In the optical band the photometric study of Dachs (1970) and Dachs \& Kabus (1989) have shown a higher number of stars and a larger fraction of peculiar Ap stars than the Pleiades. Episodes of subsequent star formation, due to passages across the Galactic Plane or interaction with the Gum Nebula, have been also speculated by Dachs \& Kabus (1989) in order to explain younger blue stragglers in this cluster. More recently, the deep photometric survey of Jeffries et al. (2001) allowed one to trace the cluster sequence down to $V \simeq 20$ and to build an optical catalog of 1254 photometric members. The Mass Function of the cluster is consistent with the empirical Salpeter's power law $\mathrm{d} N / \mathrm{d} M \propto M^{-1.35}$ but with index 1.47-1.67, depending on the assumed metallicity. The chemical composition remains uncertain for this cluster: while photometry suggests a significant underabundance of metals $([\mathrm{Fe} / \mathrm{H}]=-0.3$, Jeffries et al. 2001), more recently Terndrup et al. (2002), on the basis of high resolution spectroscopy of two stars, strongly suggested a more solar-like metallicity for NGC 2516. Furthermore, these authors conclude that an age of $150 \mathrm{Myr}$ and a distance modulus of $7.93 \mathrm{mag}$ (i.e. a distance of $\sim 385.5 \mathrm{pc}$ ) are more appropriate.

In the X-ray band Jeffries et al. (1997) and Micela et al. (2000) analyzed ROSAT PSPC and HRI observations leading to 47 detections among cluster stars with spectral type from B2 down to K. The G- and K-type stars of the cluster appeared under-luminous when compared to the Pleiades suggesting the low metallicity of the cluster (estimated at that time by photometry) to have a role in determining the X-ray emission. With the advent of Chandra and XMM-Newton missions, the cluster has been studied by Harnden et al. (2001), Sciortino et al. (2001) and Damiani et al. (2003), confirming the under-luminosity of solar type stars with respect to the Pleiades and reporting Xray emission from several Ap stars. In M-type stars the X-ray emission was found similar to the Pleiades. The time X-ray variability of stars in NGC 2516 has been studied by Wolk et al. (2004) with Chandra observations over a 2 year time span.

Unfortunately, cluster membership is determined only through photometry, since cluster proper motion is close to the solar value, making it very difficult to obtain reliable astrometric membership determination. Therefore the low X-ray emission level in solar type stars could be attributed to both a significant contamination of the cluster sample or to a real effect, related to the slightly older age of NGC 2516 with respect to the Pleiades.

In the present paper we report a deep X-ray survey of NGC 2516 obtained through a series of observations with the XMM-Newton satellite. Our aim is to determine with the highest sensitivity the X-ray emission of the cluster. In particular we focus on the X-ray properties of solar type stars of this cluster and their comparison with those of the very similar and well studied Pleiades. The structure of the paper is as follows: in Sect. 2 we present the data and the analysis method. In Sect. 3 we discuss the spectral properties and the X-ray emission level of solar type stars. In Sect. 4 we compare the X-ray luminosities from XMM-Newton with those of Chandra and ROSAT. Sect. 5 gives a discussion on other stars suggested as possible new members of the cluster, based on X-ray emission and optical photometry. In Sect. 6 we give a brief summary of relevant results.

\section{The observations and the data analysis}

NGC 2516 has been observed several times with XMM-Newton during the first two years of satellite operations for calibration purposes. Table 1 summarizes the relevant information of the observations we have used. They span a period of 19 months with exposure times between 10 and $20 \mathrm{ks}$. All of these observations have been performed with the thick filter. Figure 1 shows the summed EPIC exposures (left panel) and the optical image of the same region (right panel).

The data of each EPIC detector (MOS 1, 2 and pn) have been reduced with SAS software in order to obtain tables of photons with calibrated astrometry, arrival times and energies. Subsequently we have chosen only events in the EPIC field of view, with energies in the $0.3-7.9 \mathrm{keV}$ band, and that have triggered up to four pixels simultaneously. The data have been screened from high background rate intervals by maximizing the signal-to-noise ratio of weak sources. Figure 2 shows an example of the time screening procedure for EPIC pn of 

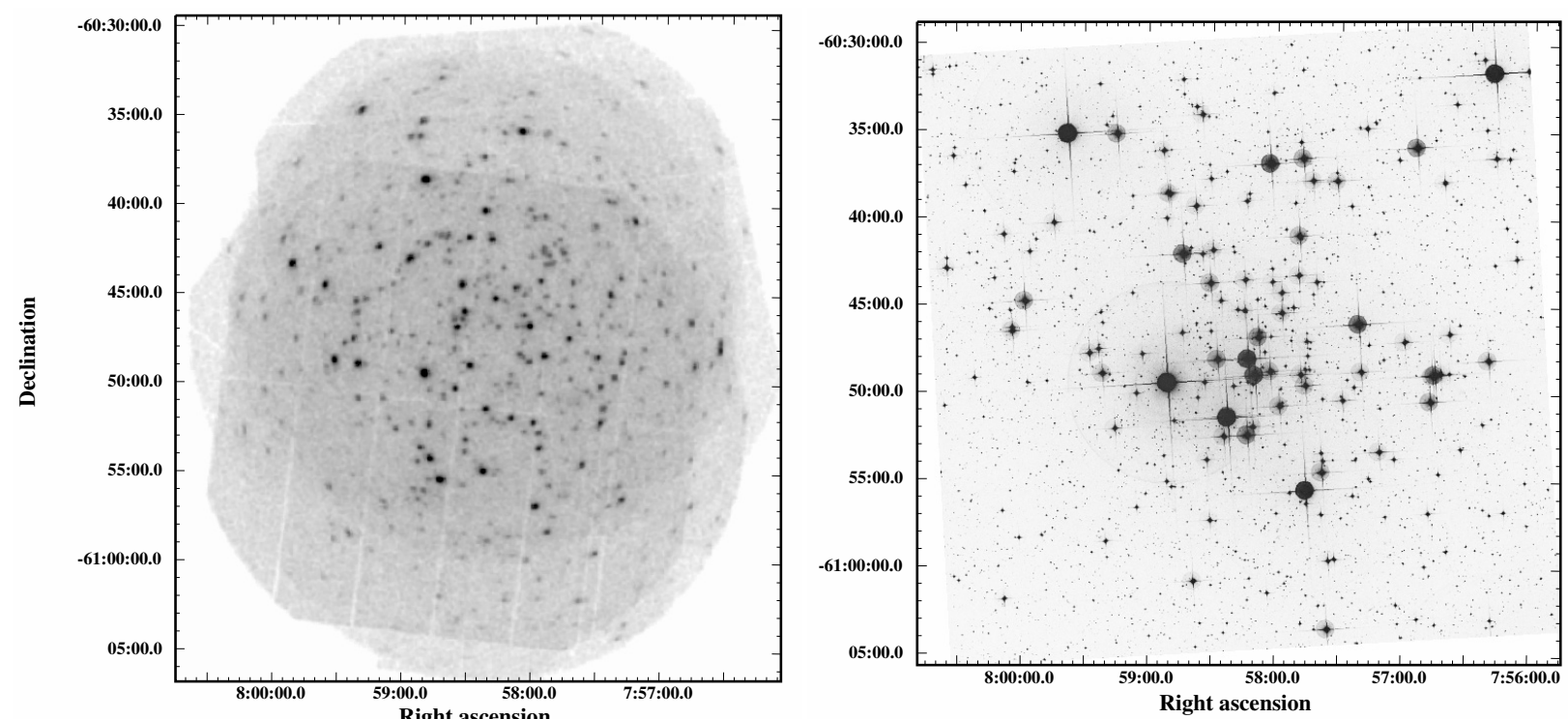

Fig. 1. X-ray (left panel) and optical (right panel) images of the NGC 2516 cluster. The X-ray survey covers a sky region of $\sim 18^{\prime} \times 25^{\prime}$ and contains 593 cluster stars (cf. Sect. 2.1).
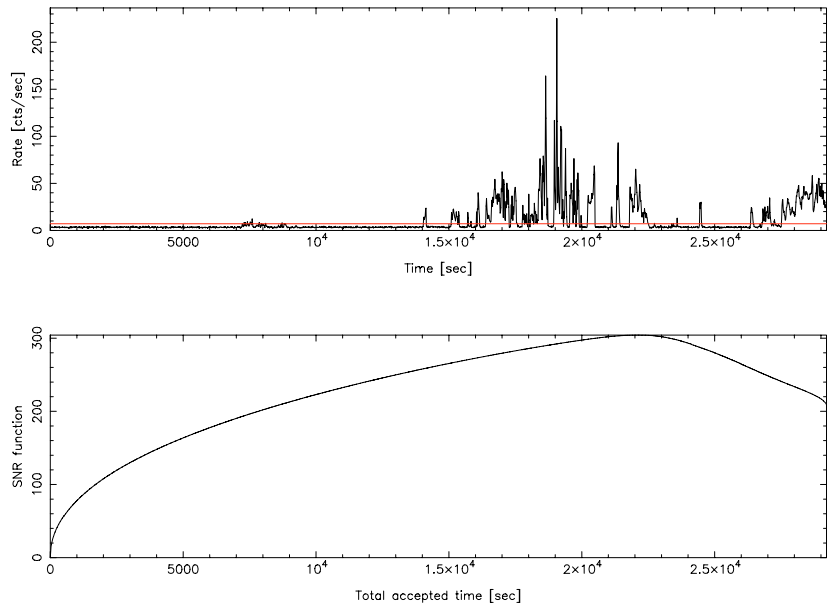

Fig. 2. Light curve for MOS 1 of EPIC observation during the XMM orbit 0092 (top panel); the horizontal line is the threshold rate that corresponds to filter the event times in order to maximize the $\mathrm{S} / \mathrm{N}$ function, plotted in the bottom panel as a function of the total accepted times.

observation during the orbit 0092 , the worst case in our set of observations. The light curve of all photons is plotted in the top panel. Spikes are due to intervals of high background. The bottom panel shows the signal to noise ratio as a function of time. We have chosen a rate threshold that filters only the time intervals yielding the maximum signal to noise ratio in the filtered image in order to optimize the detection of the weakest sources. The derived fraction of exposure for each observation (pn exposures only) is reported in Table 1.

Source detection and X-ray photometry derived from the sum of all the MOS and pn exposures have been obtained with PWXDetect code, developed at the INAF - Osservatorio Astronomico di Palermo, derived from the original ROSAT code for source detection (Damiani et al. 1997a,b) and based on the analysis of the wavelet transform of the count rate image.
It allows one to combine data from different EPIC detectors taken in different observations in order to achieve the deepest sensitivity. The code provides estimates of the positions, total counts, rates, effective exposure times and significance of each detection.

We have chosen MOS 1 as a reference detector to evaluate rates, fluxes and luminosities. The scaling factor between MOS 1, MOS 2 and pn depends on the source spectrum, the filter used during observation and the chosen energy band. In order to properly scale the rates of pn to MOS1 despite the different efficiency, we have used the ratios of source count rates, as deduced by photons accumulated in a $15^{\prime \prime}$ radius circular region at the source positions. In this way we directly use the data to find the relative instrument efficiencies without any hypotheses about the source spectrum. The rates in the summed image are equivalent to MOS 1 count rates (CR) according to the following formula:

$$
\begin{aligned}
\mathrm{CR}_{\text {sum }}= & \Sigma_{\mathrm{MOS} 1} \mathrm{CR}_{\mathrm{MOS} 1} \\
& +\Sigma_{\mathrm{MOS} 2} \mathrm{CR}_{\mathrm{MOS} 2} \cdot r_{\mathrm{MOS} 2 / \mathrm{MOS} 1}+\Sigma \mathrm{CR}_{\mathrm{pn}} \cdot r_{\mathrm{pn} / \mathrm{MOS} 1}
\end{aligned}
$$

where the medians of the scaling factors $r_{\text {MOS2/MOS1 }}$ and pn/MOS1 are respectively $\sim 1$ and $\sim 3.6$, as evaluated from source count rates measured by different detectors. The exposure times used to calculate CRs are obtained from exposure maps, and thus they take into account any spatial nonuniformities due to vignetting, RGS grating obscuration, chip geometry etc.

In the combined EPIC datasets we have detected 431 X-ray sources with a significance level greater than $5.0 \sigma$, which should lead statistically to at most one spurious source in the field of view. The threshold has been calibrated by applying the source detection algorithm to 500 simulated datasets of only background photons. In Table A.1 we report the list of sources and their properties. 


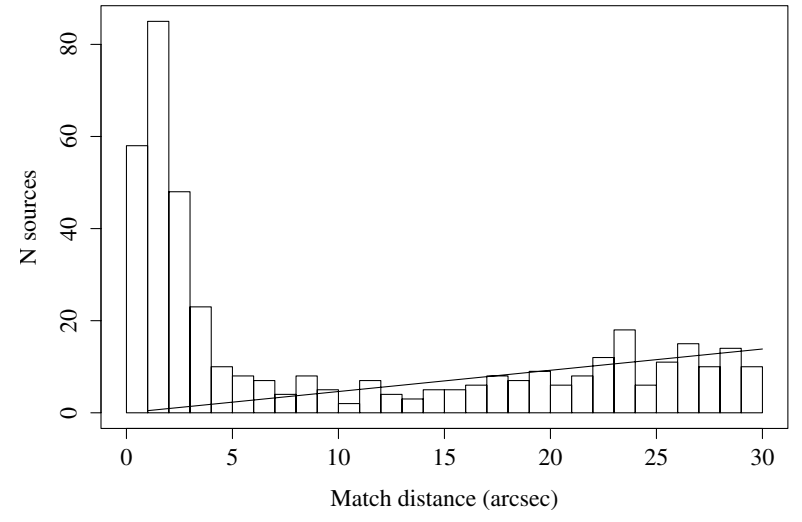

Fig. 3. Histogram of offsets between X-ray sources and the optical members positions of NGC 2516. The line traces the distribution of expected spurious matches.

\subsection{X-rays from NGC 2516: fluxes and luminosities}

The optical catalog of NGC 2516 was built from the list of 1254 photometric members compiled by Jeffries et al. (2001). The member selection is based on the photometry in the $B, V$ and $I$ bands, and 551 stars of this catalog lie in the combined field of view of the 6 XMM observations. We have added to this catalog 42 cluster stars from Dachs \& Kabus (1989) brighter than $V=9.7$, not present in the Jeffries et al. (2001) sample. This sample covers a $\sim 58^{\prime} \times 54^{\prime}$ sky region, whereas the combined EPIC image covers only about $18^{\prime} \times 25^{\prime}$, centered on the core of the cluster (cf. Fig. 1). The number of cluster stars in the surveyed area is 593. We will refer to the stars of this catalog as JTH with a running number, while for the optically bright stars we will also use the DK acronym from Dachs \& Kabus (1989).

We have matched the positions of X-ray sources with the optical coordinates of the composite catalog. No significant systematic offsets were found between optical and X-ray astrometry. By studying the distribution of the distances of matched sources, we have chosen a maximun identification radius of 7" where the number of observed matches is similar to the number of expected chance identifications. We can estimate the expected number of chance matches uncorrelated with true cluster members at a given threshold distance by taking into account the star density of the optical catalog in the EPIC field of view and the number of X-ray detections to be matched; it yields 12 false matches at the chosen 7 " radius, by integrating the line traced in Fig. 3. The value of the adopted radius does not change significantly when matching the $\mathrm{X}$-ray sources at small and large off axis positions. A list of 239 matches with the optical catalog was obtained; five X-ray sources were doubly matched with close pairs in the optical catalog; 354 cluster members in the surveyed area remain undetected in X-rays and thus we have calculated upper limits to their X-ray count rates, fluxes and luminosities at the chosen detection threshold at the positions of undetected stars. Tables B.1 and C.1 list the detected stars and the upper limits for the cluster stars, respectively.

We have estimated a conversion factor (CF) in the 0.3-7.9 keV band between count rates (in units of the MOS 1 instrument, as obtained by the detection code) and unabsorbed fluxes, derived by the spectral analysis of the bright X-ray sources of the cluster (see Sect. 2.3); the resulting CF, used for all the cluster X-ray sources, was $9.5 \times 10^{-12} \mathrm{erg} \mathrm{cnt}^{-1} \mathrm{~cm}^{-2}$. The 3 sigma uncertainty on the CF takes into account the dependence on the spectrum hardness and is $\pm 14 \%$; it would systematically shift fluxes and luminosities by +0.06 and -0.07 dex, respectively.

To obtain the luminosities we assumed a distance to the cluster of $387 \mathrm{pc}$, as adopted by Jeffries et al. (1997) and Damiani et al. (2003). This distance is slightly larger than the Hipparcos satellite estimate ( $346 \mathrm{pc}$ ); by assuming this latter the luminosities would decrease by -0.05 dex. Table 2 reports the number and the rate of detections for each spectral type (indeed for each range of $B-V$ or $V-I$ color as reported in the header of the table), the number of stars in the field of view, the medians of X-ray luminosities, also after correction for non members contamination (see Sect. 3.3) and the minimum of detected luminosity. The weakest source of the cluster is a M-type star, with a X-ray flux equal to $1.2 \times 10^{-15} \mathrm{erg} \mathrm{s}^{-1} \mathrm{~cm}^{-2}$ and luminosity of $2.2 \times 10^{28} \mathrm{erg} \mathrm{s}^{-1}$. For comparison the Chandra survey of Damiani et al. (2003) led to a minimum detected flux among cluster sources of $2.75 \times 10^{-15} \mathrm{erg} \mathrm{s}^{-1} \mathrm{~cm}^{-2}$ in a very similar band. The choice of the $\mathrm{CF}$ and the distance does not result in a systematic difference between the luminosities obtained in the present survey and that of Damiani et al. (2003) conducted with Chandra (cf. Fig. 9); in Sect. 4 we discuss in detail the comparison of luminosities estimated with Chandra and XMM-Newton.

\subsection{Uncertainties on $X$-ray fluxes and luminosities}

We summarize here all the statistical and systematic errors that could affect our estimates of fluxes and luminosities. Count rates have relative errors in the 5\%-30\% range (as reported in Table A.1). The CF has a $14 \%$ uncertainty at $3 \sigma$ level from fit of fluxes vs. count rates, essentially due to the effect of source spectrum temperature on CF. The scaling of pn to MOS introduces an uncertainty of $\sim 11 \%$ i.e. fluxes and luminosities can be systematically higher or lower by -0.03 or +0.015 dex, respectively. The different distance estimates given in the literature should systematically shift the X-ray luminosities by -0.05 (346 pc, Hipparcos distance) or +0.022 (for a distance of $407 \mathrm{pc}$, Terndrup et al. 2002). All these sources of uncertainties could balance themselves, the errors in Table B.1 may suffer an additional systematic uncertainty of $\sim \pm 0.07$ dex in flux and \pm 0.08 dex in luminosities.

\subsection{Spectral analysis}

We used EPIC data to investigate the spectral properties of the NGC 2516 stellar coronae in terms of relevant temperatures and emission measures. The pn has the highest efficiency in collecting X-ray photons among the three EPIC instruments, hence we have used the data from each pn exposure by simultaneously fitting the X-ray spectra observed in different 
Table 2. Number of X-ray detections, number of stars in the EPIC field of view, medians of $\log L_{\mathrm{X}}$ distributions (we report also the values corrected for non member contaminants in G-, K- and M-type stars, see Sect. 3.3) and minimum detected luminosity for B, A-, F-, G-, K- and M-type stars of NGC 2516. The selection of the spectral types is reported in the table header. In cases where the rate of detection is $<50 \%$ only an upper limit to the median is reported.

\begin{tabular}{lllllll}
\hline \hline Sp. Type & $\mathrm{B}$ & $\mathrm{A}$ & $\mathrm{F}$ & $\mathrm{G}$ & $\mathrm{K}$ & $\mathrm{M}$ \\
& $(B-V)_{0}<0$ & $0 \leq(B-V)_{0} \leq 0.3$ & $0.3 \leq(B-V)_{0} \leq 0.5$ & $0.5 \leq(B-V)_{0} \leq 0.8^{a}$ & $0.93 \leq(V-I)_{0} \leq 2.2$ & $2.2 \leq(V-I)_{0} \leq 5.0$ \\
\hline$N_{\text {det }} / N_{\text {tot }}$ & $13 / 38$ & $16 / 49$ & $24 / 37$ & $52 / 89$ & $74 / 182$ & $59 / 194$ \\
Detect. Rate & $34 \%$ & $33 \%$ & $65 \%$ & $58 \%$ & $41 \%$ & $30 \%$ \\
$\log L_{\mathrm{X}}$ median & $\leq 28.62$ & $\leq 28.44$ & 29.19 & 28.95 & $\leq 28.70$ & $\leq 28.62$ \\
$\log L_{\mathrm{X}}$ med.nocont. & $\ldots$ & $\ldots$ & $\ldots$ & 29.04 & $\leq 28.82$ & $\leq 28.68$ \\
Min Det $\log L_{\mathrm{X}}$ & 28.85 & 28.60 & 28.71 & 28.55 & 28.5 & 28.34 \\
\hline
\end{tabular}

${ }^{a}$ Combined with: $(V-I)_{0} \leq 0.93$.

Table 3. Temperatures and emission measures estimated from global fits of coronal spectra. Models with 1-T or 2-T components have been used, errors refer to $10 \%-90 \% \chi^{2}$ confidence range.

\begin{tabular}{|c|c|c|c|c|c|c|}
\hline Num & $\overline{B-V}$ & $\overline{T_{1}(\mathrm{keV})}$ & $\bar{T} T_{2}(\mathrm{keV})$ & $\log \mathrm{EM}_{1}\left(\mathrm{~cm}^{-3}\right)$ & $\log \mathrm{EM}_{2}\left(\mathrm{~cm}^{-3}\right)$ & $\overline{\chi^{2} / \text { d.o.f. }}$ \\
\hline 9465 & 0.818 & $0.72_{-0.08}^{0.07}$ & $1.69_{-0.3}^{0.5}$ & $52.93_{-0.17}^{0.04}$ & $53.06_{-0.17}^{0.02}$ & $2.7 / 88$ \\
\hline 9676 & 0.903 & $0.34_{-0.07}^{0.09}$ & $1.14_{-0.11}^{0.13}$ & $52.79_{-0.2}^{0.02}$ & $52.94_{-0.13}^{0}$ & $2.4 / 95$ \\
\hline 7678 & 0.928 & $0.37_{-0.08}^{0.13}$ & $1.59_{-0.3}^{0.7}$ & $52.6_{-0.24}^{0.04}$ & $52.63_{-0.18}^{0.03}$ & $2.3 / 25$ \\
\hline 7782 & 1.417 & $0.28_{-0.09}^{0.1}$ & $1.25_{-0.3}^{0.5}$ & $52.69_{-0.5}^{0.05}$ & $52.99_{-0.13}^{0.04}$ & $4.5 / 7$ \\
\hline 9175 & 0.585 & $0.66_{-0.07}^{0.09}$ & - & $53.2_{-0.2}^{0.1}$ & $-\quad-0.10$ & $3.5 / 15$ \\
\hline 8893 & 1.433 & $0.32_{-0.06}^{0.3}$ & $1.29_{-0.15}^{0.15}$ & $52.51_{-0.2}^{0.08}$ & $52.73_{-0.1}^{0.03}$ & $2.3 / 42$ \\
\hline 11233 & 0.834 & $0.6_{-0.2}^{0.08}$ & $1.2_{-0.2}^{0.2 .15}$ & $52.91_{-0.14}^{0.09}$ & $52.86_{-0.16}^{-0.06}$ & $1.3 / 55$ \\
\hline 6649 & 0.817 & $0.56_{-0.25}^{0.24}$ & $1.3_{-0.2}^{0.3}$ & $52.22_{-0.2}^{0.4}$ & $52.78_{-0.2}^{0.08}$ & $1.1 / 23$ \\
\hline 7585 & 0.719 & $0.6_{-0.2}^{0.07}$ & $2.04_{-0.6}^{1.1}$ & $52.68_{-0.3}^{0.07}$ & $52.68_{-0.13}^{0.04}$ & $1.4 / 26$ \\
\hline 4598 & 0.861 & $0.7_{-0.1}^{0.1}$ & $2.1_{-0.5}^{0.9}$ & $52.96_{-0.2}^{0.04}$ & $53.16_{-0.21}^{0.1}$ & $1.3 / 28$ \\
\hline 9140 & 0.736 & $0.74_{-0.14}^{0.07}$ & $1.7_{-0.7}^{2.5}$ & $52.85_{-0.2}^{0.04}$ & $52.54_{-0.6}^{0.2}$ & $1.35 / 26$ \\
\hline 7864 & 0.603 & $0.7_{-0.4}^{0.4}$ & $1.6_{-0.5}^{8.4}$ & $52.5_{-0.7}^{0.2}$ & $52.8_{-0.5}^{0.09}$ & $0.3 / 6$ \\
\hline 8997 & 0.981 & $0.8_{-0.1}^{0.2}$ & - & $52.85_{-0.11}^{-0.01}$ & - & $2.0 / 38$ \\
\hline 8458 & 0.533 & $0.54_{-0.06}^{0.04}$ & - & $52.92_{-0.1}^{-0.01}$ & - & $1.2 / 47$ \\
\hline 8099 & 0.56 & $0.61_{-0.05}^{0.05}$ & - & $52.81_{-0.11}^{-0.02}$ & - & $2.1 / 20$ \\
\hline 9054 & 0.691 & $0.32_{-0.09}^{0.06}$ & $0.9_{-0.15}^{0.09}$ & $52.73_{-0.1}^{0.1}$ & $52.65_{-0.06}^{0.09}$ & $1.6 / 49$ \\
\hline 7667 & 0.195 & $0.60_{-0.04}^{0.4}$ & - & $52.66_{-0.05}^{0.05}$ & - & $1.7 / 26$ \\
\hline 10046 & 0.99 & $0.31_{-0.05}^{0.05}$ & $1.5_{-0.3}^{0.3}$ & $52.81_{-0.3}^{-0.01}$ & $52.93_{-0.1}^{0.02}$ & $0.7 / 16$ \\
\hline 8529 & 0.583 & $0.64_{-0.04}^{0.06}$ & - & $52.85_{-0.1}^{-0.03}$ & - & $2.1 / 24$ \\
\hline 8886 & 0.113 & $0.32_{-0.2}^{0.17}$ & $3.5_{-1.0}^{1.7}$ & $52.3_{-0.7}^{1.3}$ & $52.9_{-0.1}^{0.1}$ & $0.7 / 22$ \\
\hline 6029 & 0.674 & $0.4_{-0.1}^{0.2}$ & $1.3_{-0.3}^{1.1}$ & $52.92_{-0.2}^{0.05}$ & $52.7_{-0.3}^{0.01}$ & $1.8 / 19$ \\
\hline 8634 & 0.943 & $0.38_{-0.07}^{0.14}$ & $1.21_{-0.15}^{0.14}$ & $52.79_{-0.2}^{0.01}$ & $52.92_{-0.16}^{-0.02}$ & $1.0 / 34$ \\
\hline 9835 & 0.988 & $0.14_{-0.13}^{0.11}$ & $0.94_{-0.12}^{0.13}$ & $52.9_{-0.6}^{1.2 .2}$ & $52.91_{-0.2}^{-0.03}$ & $2.4 / 14$ \\
\hline 10817 & 0.525 & $0.59_{-0.17}^{0.16}$ & $11_{-6}^{6}$ & $53.02_{-0.3}^{0.05}$ & $53.48_{-0.07}^{0.05}$ & $1.4 / 16$ \\
\hline 8641 & 1.063 & $0.37_{-0.07}^{0.13}$ & $0.99_{-0.09}^{0.09}$ & $52.91_{-0.23}^{0.02}$ & $52.99_{-0.12}^{0.01}$ & $1.4 / 64$ \\
\hline 7743 & 0.655 & $0.73_{-0.09}^{0.07}$ & - & $52.82_{-0.13}^{-0.01}$ & - & $1.0 / 12$ \\
\hline DK23 & 0.06 & $0.56_{-0.12}^{0.06}$ & - & $51.6_{-0.2}^{1.0}$ & - & $1.6 / 21$ \\
\hline DK44B & -0.02 & $0.73_{-0.11}^{0.07}$ & - & $52.68_{-0.05}^{0.05}$ & - & $1.5 / 24$ \\
\hline DK56 & -0.09 & $0.8_{-0.1}^{0.2}$ & $3_{-0.4}^{0.7}$ & $52.56_{-0.2}^{0.09}$ & $53.29_{-0.04}^{0.04}$ & $0.9 / 99$ \\
\hline DK73 & 0.02 & $0.6_{-0.07}^{0.1}$ & - & $52.69_{-0.05}^{0.07}$ & - & $1.5 / 19$ \\
\hline DK62 & 0.03 & $0.61_{-0.2}^{0.05}$ & $1.4_{-0.3}^{0.5}$ & $52.76_{-0.18}^{0.08}$ & $52.81_{-0.17}^{0.11}$ & $1.5 / 39$ \\
\hline 6689 & 0.329 & $0.73_{-0.11}^{0.12}$ & - & $53.43_{-0.16}^{0.11}$ & - & $1.4 / 10$ \\
\hline 9852 & 0.659 & $0.54_{-0.07}^{0.06}$ & - & $53.0_{-0.5}^{0.2}$ & - & $2.2 / 19$ \\
\hline 7650 & 0.62 & $0.6_{-0.1}^{0.1}$ & - & $53.0_{-0.3}^{0.2}$ & - & $1.1 / 6$ \\
\hline 8660 & 0.571 & $0.59_{-0.1}^{0.08}$ & - & $53.1_{-0.2}^{0.3}$ & - & $3.8 / 12$ \\
\hline 10040 & 1.034 & $0.76_{-0.1}^{0.09}$ & - & $53.22_{-0.15}^{0.15}$ & - & $0.9 / 12$ \\
\hline 9882 & 0.185 & $0.65_{-0.06}^{0.06}$ & - & $53.1_{-0.3}^{0.2}$ & - & $1.1 / 13$ \\
\hline 8967 & 0.996 & $0.75_{-0.17}^{0.17}$ & - & $53.24_{-0.17}^{0.2}$ & - & $0.7 / 5$ \\
\hline
\end{tabular}


exposures ${ }^{1}$. In order to perform a spectral analysis with a global fitting technique. we have accumulated the spectra for sources with more than 500 total counts in the sum of datasets. For 31 sources with more than 1000 counts in the summed image, the photons were extracted within a $30^{\prime \prime}$ radius circular region centered at the position of the source, and the background spectrum was accumulated in annuli (with radii of $35^{\prime \prime}$ and $45^{\prime \prime}$, respectively).

For 7 sources with counts between 500 and 1000 total counts we chose to accumulate counts in a $20^{\prime \prime}$ radius region and to fit spectra without background subtraction, considering that background photons are reduced to $44 \%$ in this area with respect to the area of radius $30^{\prime \prime}$, whereas the source encircled energy is reduced from $80 \%$ to $\simeq 70 \%$. In this way we expect to reduce the contamination of the background spectrum and to save most of the source photons without increasing the uncertainties due to background subtraction. We have tested this procedure in a few cases finding that the background subtraction increases the relative error of fitted parameters by more than $30 \%$, while the best fit temperature slightly decreases within the uncertainty range. The background photons are $\sim 60 \pm 30$ in the $20^{\prime \prime}$ extraction area, the actual number depending on the source off-axis distance. We have also included sources with only 500 counts to reduce possible biases introduced by considering only higher count statistics, hence higher activity stars.

The spectra of sources with the highest statistics were fit in most cases with a sum of two APEC models plus photoelectric absorption. After having tried models with the absorption and the abundance as free parameters, we fix the column density value to $N_{\mathrm{H}}=8 \times 10^{20} \mathrm{~cm}^{-2}$, consistent with estimates from photometry (Jeffries et al. 2001), and the abundances to those at $0.3 Z_{\odot}$ because we noticed that under-solar abundances are always obtained, leaving thus as free parameters the two temperatures and emission measures. The spectra of low statistics sources were fit with one APEC model, with as free parameters the temperature, abundance and emission measure and a fixed absorption at $N_{\mathrm{H}}=8 \times 10^{20} \mathrm{~cm}^{-2}$, as chosen before. Results from spectral fitting are reported in Table 3 and discussed in Sect. 3.2; errors refer to the $10 \%-90 \% \chi^{2}$ confidence range.

\section{Discussion}

\subsection{X-ray emission along the main sequence}

Figure 4 shows the color-magnitude diagrams (CMD) in $B, V$, $I$ bands for the photometric cluster members. Hotter stars are shown only in the left panel (CMD with $B-V$ ) while cooler stars with unreliable $B-V$ are shown only in the CMD with $V-I$ color index. The X-ray detections span the Main Sequence of the cluster from B type stars down to cool, low mass M-type stars.

\footnotetext{
${ }^{1}$ In some cases we have excluded spectra with very few photons so for each source we do not always fit simultaneously all the spectra from the six observations.
}
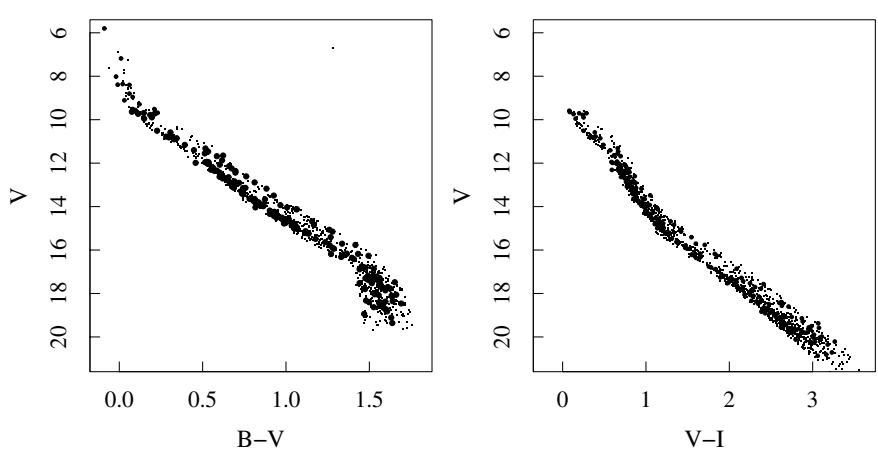

Fig. 4. Color-magnitude diagrams in $B, V, I$ bands of NGC 2516 stars (small dots) from Jeffries et al. (2001) and Dachs \& Kabus (1989). Large dots are X-ray detected stars.

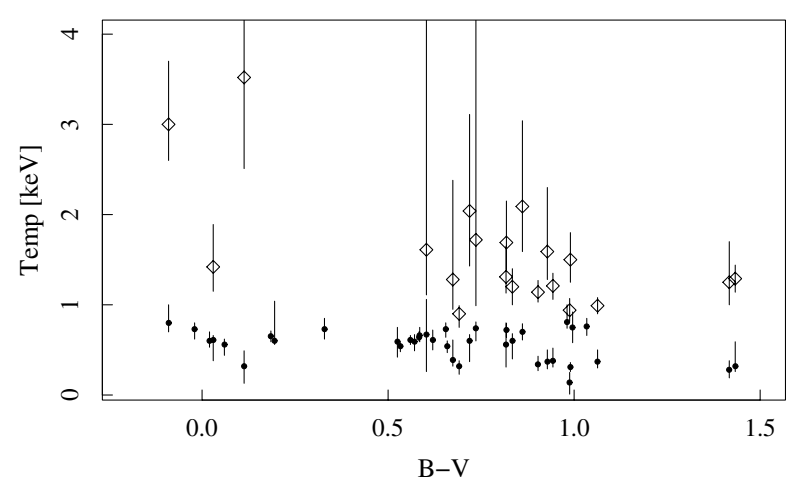

Fig. 5. Coronal temperatures as a function of $B-V$ color. Filled dots refer to the cool component whereas empty diamonds refer to the hot component.

\subsection{X-ray spectral properties}

The sample of stars for which we have analyzed the spectra contains by solar G- and K- type stars and hot, massive B- and early A- type stars. We were unable to analyze the spectra of stars with $B-V \sim 0.4$ (late-A spectral type stars) because of their lower X-ray fluxes and hence spectra with poor statistical significance. The models we used are too simple and unrealistic; a distribution of plasma temperatures would be more realistic. However we can determine the dominant temperatures of the coronae as is usually done with low resolution X-ray spectra.

In Fig. 5 we plot the temperatures derived from global fitting as a function of $B-V$ color. We were able to fit most of the spectra of solar type stars with 2 temperature models. High energy tails of spectra have very few photons, consequently hot components have been estimated with large errors. The temperatures are in the $0.3-0.7 \mathrm{keV}$ and $1.0-2.0 \mathrm{keV}$ range, and are similar to those detected in the coronae of Pleiades and Blanco 1 open clusters, which are nearly coeval to NGC 2516 (Briggs \& Pye 2003; Pillitteri et al. 2004). The ratio of hot to cool emission measures in 2-T models suggests that the two thermal components are comparable, the ratio being between 0.5 and 3.0 with a median of 1.3 .

Spectra of all but 3 early type stars $(B-V \lesssim 0.5)$ have low count statistics: we may obtain a good fit of them with 1-T models. In this case the temperatures lie around $0.6 \mathrm{keV}$, 
suggesting that the spectra are softer than those of solar mass stars.

The star DK 56 (a binary system with a B2V star and an unresolved late type companion) was bright enough in each observation to fit its spectrum with a $2-\mathrm{T}$ model. We found a hot spectrum with temperatures of 0.8 and $3.0 \mathrm{keV}$ and a spectrum shape similar to those of solar type stars. In the case of a wind driven X-ray emission, typical of massive stars, the spectrum is expected to be softer than in the case of coronae of solar mass stars. Therefore, based on the spectrum shape and temperatures, we suggest that the X-ray emission of DK 56 is likely due to or strongly affected by the late type companion. This is a conclusion opposite to that of Jeffries et al. (1997) who, on the basis of its high X-ray luminosity, attributed all the $\mathrm{X}$-ray emission of DK56 to the massive primary. This system is a blue straggler of the cluster, younger than $25 \mathrm{Myr}$ (Dachs $\&$ Kabus 1989). Based on this age estimate, these authors discussed the hypothesis of subsequent episodes of star formation in NGC 2516 determined by the crossing of the cluster through the Galactic Plane or the Gum Nebula. Given this age estimate, the high X-ray luminosity of the order of $10^{30} \mathrm{erg} \mathrm{s}^{-1}$ is not an atypical value for solar-type stars of $25 \mathrm{Myr}$ or less.

\subsection{The X-ray luminosities of NGC 2516 late type stars}

We have calculated the Kaplan-Meier estimators (Feigelson \& Nelson 1985) of the distribution of X-ray luminosities for F, G, $\mathrm{K}$ and $\mathrm{M}$ spectral types. The distributions take into account the the upper limits in $\log L_{X}$ that censorize the sample. When the lowest values are upper limits the distribution does not reach unity. Figure 6 shows the X-ray distribution functions for F-, G-, K- and M-type stars of NGC 2516 (solid lines); for comparison we have added the ROSAT data of Pleiades (Micela et al. 1999, dotted line) and the distribution considering only detections of NGC 2516 (solid gray line). This is an upper bound to the true distribution which must include undetected members. The Kaplan-Meier estimators of Pleiades and NGC 2516 are different $^{2}$. In particular, the luminosities of NGC 2516 of G-, $\mathrm{K}$ - and M- type stars are systematically lower than those of the Pleiades, the difference being larger for G- and K-type stars where the contamination of the optical catalog is higher than in the other spectral ranges, as stated by Jeffries et al. (2001). Especially among K-type stars the Kaplan-Meier estimator and cumulative distribution of X-ray detections alone show the largest difference.

The contamination by less active field stars among G- and K-type stars in NGC 2516 hampers the evaluation of $L_{\mathrm{X}}$ distributions of NGC 2516. However, the X-ray luminosities of detections alone of G-, K- and M-type stars are systematically lower than the luminosities of the Pleiades at a level $\geq 99 \%$ as obtained by applying several two sample tests. The distribution of detections alone shown in Fig. 6 may be interpreted as an upper limit for the true cumulative distribution of X-ray luminosity. If we were able to take into account the upper limits of

\footnotetext{
${ }^{2}$ By applying several two sample tests, the difference between the two curves is significant at a level $\gtrsim 99.9 \%$.
}
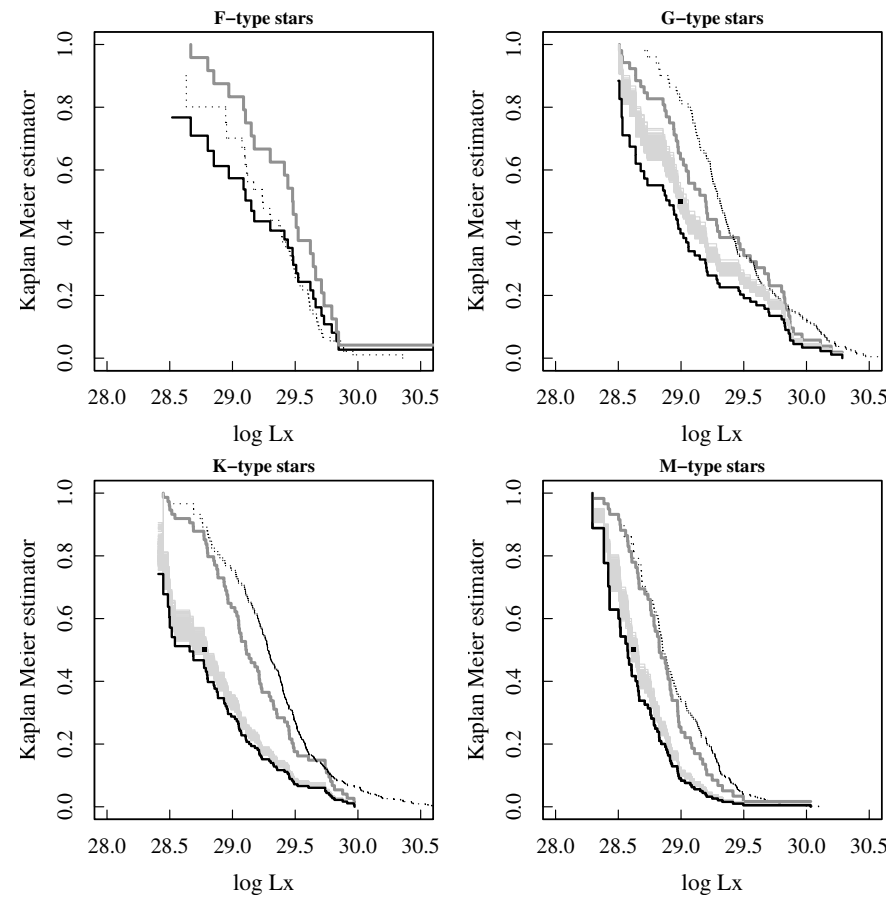

Fig. 6. Cumulative distribution functions of X-ray luminosities of NGC 2516 stars (solid black line) with different spectral types, evaluated with Kaplan Meyer estimators. Dotted lines are the distributions of Pleiades (Micela et al. 1999). Solid gray lines in G-, K- and M-type stars are the distribution of detections alone. The gray area in G-, Kand M-type panels marks the envelop of simulated Kaplan-Meier estimators after statistical correction for contaminants; the black square inside each area is the average of their medians.

only true cluster members, the Kaplan-Meier estimators should be at lower luminosities with respect to the distribution of detections alone.

We have evaluated how many field stars are expected to fall among our X-ray detections. We focused on the main sequence field stars expected to be in a volume determined by the photometric selection of the cluster Main Sequence and the sky area of our survey. By using the stellar density given in Cox (2000), Table 19.14 (valid for stellar density on the galactic plane, NGC 2516 is $\sim 100$ pc above the Galactic Plane) we estimated 11 and $19 \mathrm{G}$ and K-type main sequence field stars in that volume and not related to the cluster.

From the X-ray luminosity distributions reported in Schmitt et al. (1995) and Schmitt (1997) G-type field stars have $\log L_{\mathrm{X}} /\left(\mathrm{erg} \mathrm{s}^{-1}\right)$ in the range 26.5-29.5 with median 27.3; K-type stars occupy a narrower range (27.1-28.4) with a median of $\log L_{\mathrm{X}} /\left(\mathrm{erg} \mathrm{s}^{-1}\right)=27.6$. M-type stars in the solar neighborhood have luminosities $25.5 \leq \log L_{X} /\left(\mathrm{erg} \mathrm{s}^{-1}\right) \leq 29.1$ and a median of $\log L_{\mathrm{X}} /\left(\mathrm{erg} \mathrm{s}^{-1}\right) \sim 27$. Hence we estimated that less than 3 field stars in each spectral type should have been detected among the photometric sample (at the same time a sample of 10 to 18 stars should be undetected in the surveyed volume). More distant giants should not be detected and thus they should not affect the distribution of detections alone. The expected field star detections are thus very few compared to the number of detected cluster members in each sample 
(52 G-, $74 \mathrm{~K}$ - and 59 M-type detections), thus the distribution of detections alone should not be affected by field stars.

We have taken into account the expected fraction of contaminants given by Jeffries et al. (2001) in order to estimate unbiased X-ray luminosity distribution functions of NGC 2516 G-, K- and M-type stars. By following the method used in Damiani et al. (2003), we excluded a fraction of upper limits from the X-ray luminosity sample in each spectral type according to the membership probability and assuming that the contaminants yield only upper limits; then we calculated the Kaplan-Meier estimator. This simulation procedure was iterated 500 times producing thus the bunch of curves plotted in light gray in Fig. 6 and which define the region where the true distribution function should lie. Only in the case of G-type stars do we observe a larger difference between the curve with upper limits and the family of contaminant corrected curves. In the K- and M-type stars the large number of upper limits is less affected by the statistical correction for contaminants. In all cases the gray region is at lower $\mathrm{X}$-ray luminosities than the Pleiades cumulative distributions.

The averages of the 0.5 quantile of each simulated curve are marked in each panel with a black square; the $\log L_{X} /\left(\mathrm{erg} \mathrm{s}^{-1}\right)$ values are 29.04, 28.82 and 28.68 for G-, K- and M-type stars, respectively; the width of the gray region at the 0.5 quantile is $\sim 0.05$ dex which we assume as the uncertainty on the previous averages. Güdel (2004) and Favata \& Micela (2003) discuss X-ray emission as a function of age for stars from young stellar objects to aged open clusters and the Sun. We have plotted in Fig. 7 the medians of $\log L_{X}$ for F-, G-, K- and M-type stars reported by Güdel (2004) and our $\log L_{\mathrm{X}}$ medians (after contaminant correction) for the same spectral types as a function of age. We have assumed here an age of $140 \mathrm{Myr}$ for NGC 2516. We see a decrease of the mean level of X-ray luminosities with age, after a plateau at $\sim 10^{30} \mathrm{erg} \mathrm{s}^{-1}$ which extends from Orion and Taurus Star forming Region ages (few Myr) to Alpha Persei cluster age (50 Myr). The X-ray under-luminosity of NGC 2516 with respect to the nearly coeval Pleiades remains evident, even taking into account the statistical correction for non member contamination and any systematic uncertainty discussed in Sect. 2.2.

The causes of the different levels of luminosity of solar type stars in NGC 2516 and the Pleiades could be attributed to several factors. Rotation is a key parameter for X-ray emission (Pallavicini et al. 1981) up to rotations as fast as $v \sin i \sim 20 \mathrm{~km} \mathrm{~s}^{-1}$, when the $L_{\mathrm{X}}-$ rotation relation flattens. Furthermore, angular momentum losses due to magnetic braking link stellar rotation to age and X-ray activity. A different distribution of rotation rates in the Pleiades and NGC 2516 could explain the low X-ray luminosity observed in the latter cluster. Jeffries et al. (1998) studied the stellar rotation of solar type stars of NGC 2516, finding a lower rotational rate in F- early and G- type stars with respect to the Pleiades whereas no clear difference between these two clusters was found among K-type stars. However Terndrup et al. (2002) concluded that no significant difference in rotation distribution is present between the Pleiades and NGC 2516, if this latter is assumed slight older than Pleiades. The under-luminosity of NGC 2516 in X-rays seems to arise essentialy from the slight

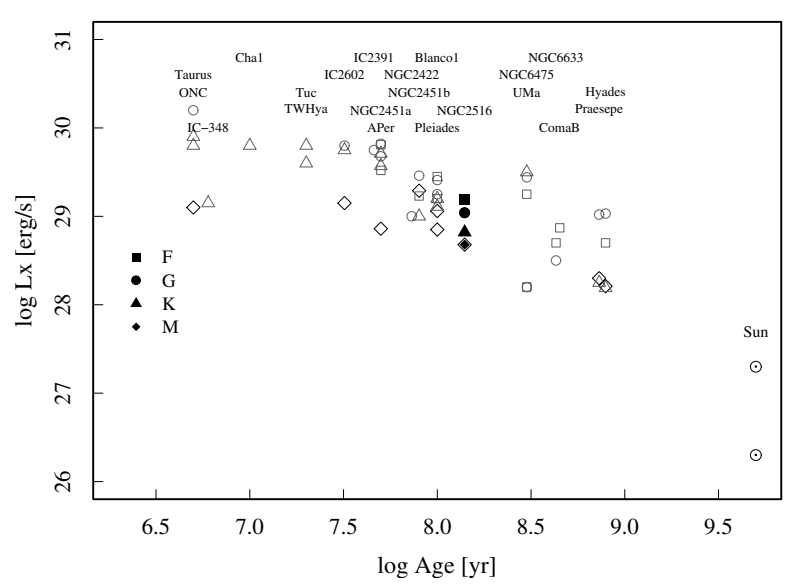

Fig. 7. $\log L_{\mathrm{X}}$ vs. age for different open clusters, star forming regions and the Sun. Open symbols: data of all but NGC 2516 clusters from Güdel (2004); solid symbols: medians of NGC 2516 F-, G-, K- and M-type stars (contaminants corrected, see. Sect. 3.3) from the present work.

difference of age between the two clusters (80-100 Myr for Pleiades, 140 Myr for NGC 2516), although the past history of NGC 2516, its location and kinematics could have played a role in determining peculiar forming conditions, perhaps different to those of the Pleiades with an influence on the X-ray emission evolution.

\section{4. $L_{x}$ to $L_{\text {bol }}$ ratio}

An indicator of activity is the ratio of X-ray to stellar bolometric luminosity, $\log L_{\mathrm{X}} / L_{\mathrm{bol}}$. Because of the dependence of $\mathrm{X}$-ray luminosity on rotation, the $\log L_{\mathrm{X}} / L_{\text {bol }}$ ratio follows a power law relation of index -2 with rotational period and the Rossby number, i.e. the ratio between rotational period and convective turnover time in non saturated stars (see Patten \& Simon 1996; Randich 2000; Pizzolato et al. 2003). ROSAT observations of young open clusters in the band $0.1-2.4 \mathrm{keV}$ have also shown that low mass stars exhibit a saturation of this ratio at the level of $\sim-3$, i.e. the X-ray luminosity reaches at most 1/1000 of bolometric luminosity of the star. Furthermore, saturation seems to occur at earlier types in younger stars ( $B-V=0.7$ or late G-type stars at the age of the Pleiades, $B-V=1$ or mid K-type stars at $220 \mathrm{Myr}$, Prosser et al. 1995).

We calculated the bolometric luminosities of NGC 2516 by interpolating $L_{\mathrm{bol}}$ as a function of $(B-V)$ based on an isochrone model with $Z=0.02$ and age of $140 \mathrm{Myr}$ (Siess et al. 2000); for stars with $(B-V)_{0} \geq 0.3$ we used $(V-I)$ color instead of $(B-V)$. Figure 8 shows $\log L_{\mathrm{X}} / L_{\mathrm{bol}}$ of NGC 2516 stars versus their $B-V_{0}$ and $V-I_{0}$ colors, with open and filled circles for single and binaries, respectively. We did not correct $L_{\mathrm{bol}}$ for unresolved binary systems. These unresolved binaries trace an upper sequence in the plots. Arrows represent upper limits and the bottom curve approximately traces the detection limit at the cluster distance $\left(\log L_{X} \sim 28.4\right)$. A spread of $\sim 1.4$ dex is observed among detections in $0 \leq B-V \leq 1.5$ where $\log L_{X} / L_{\text {bol }}$ may reach values up to -2.4 . The highest point at $\sim-1.4$ is due to a very cool star $(V-I=3.06$, id. 6160 in the Jeffries et al. 2001 catalog): however this star is very close to the edge of the 

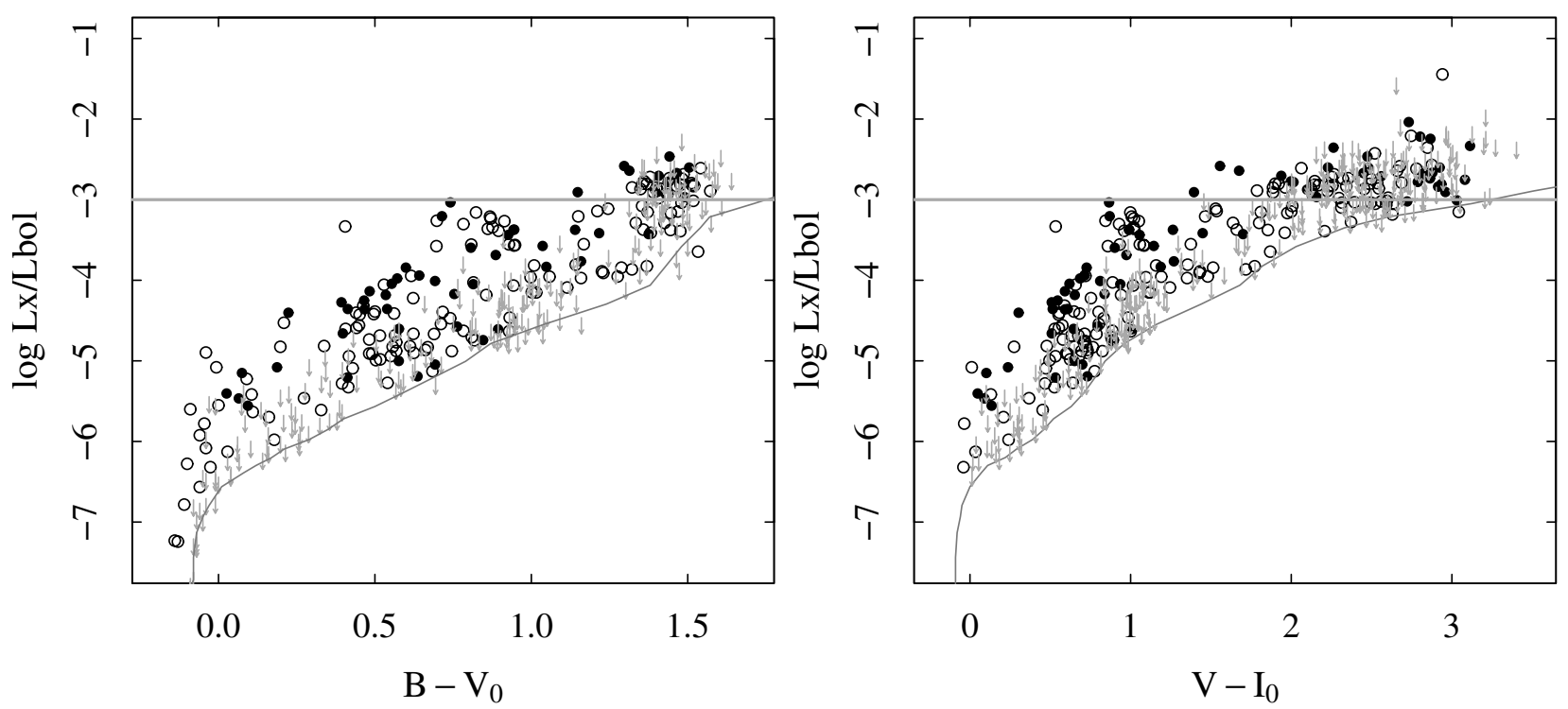

Fig. 8. Logarithmic X-ray to bolometric luminosity ratio of NGC 2516 stars. Open circles: detected single stars, filled circles: detected binaries, vertical arrows: upper limits. Lines below data points roughly trace the detection limit at the cluster distance $\left(\log L_{\mathrm{X}} \sim 28.4\right)$.

field of view and was observed only during the first observation, hence its X-ray luminosity is quite uncertain.

The $\log L_{\mathrm{X}} / L_{\text {bol }}$ ratio flattens at $(V-I)_{0} \sim 1.5$ or $(B-V)_{0} \sim$ 0.7 , which corresponds to the spectral type $\mathrm{K} 5$, according to the color-temperature calibration by Kenyon \& Hartmann (1995) and we observe a saturation value of $\log L_{\mathrm{X}} / L_{\mathrm{bol}} \sim-2.5$ instead of -3 . A comparison with $L_{\mathrm{bol}}$ used by Jeffries et al. (1997) shows that our $L_{\mathrm{bol}}$ are on average lower by $0.1 \mathrm{dex}$ for stars detected in both surveys. This difference would produce a shift toward high $\log L_{X} / L_{\text {bol }}$ ratio, while the trend and the spectral type at which the saturation of $\log L_{\mathrm{X}} / L_{\mathrm{bol}}$ occurs should not be changed.

\section{Comparison with Chandra and ROSAT surveys}

NGC 2516 has been observed several times with the Chandra satellite between August 1999 and March 2001 with different instrument setups; a deep survey was obtained by Damiani et al. (2003) combining several exposures from ACIS and HRC cameras. In that work 155 cluster stars were detected in Xray while 570 remained undetected. There are 535 stars falling both in the XMM-Newton and Chandra fields of view; out of this sample we have detected 125 stars with the EPIC camera also detected with Chandra; a further 100 stars are detected with EPIC whereas they were undetected in Chandra images. Only 17 stars are detected with Chandra but not with XMMNewton; 293 stars remain undetected in both surveys. In Fig. 9 we show the comparison of Chandra and XMM-Newton X-ray luminosities for the stars detected in at least one of these surveys. The plot shows that a scatter of a factor $\lesssim 2$ is observed in most cases; sometimes a difference of a factor 5 or more may be present also in a few massive stars (DK 55 / HD 66167, B9.5V + A0V; HD 66194 / DK 56, sp. type B2IV).

The upper limits marked by arrows in the lower right corner of Fig. 9 are stars undetected with Chandra but detected with XMM-Newton. This large number of upper limits is due to the sensitivity of our survey being a factor of $\sim 5$ higher than the Chandra one. The maximum sensitivity is comparable in both satellite instruments but in Chandra the decrease of sensitivity at large off-axis angles is more marked. Both the large effective area of XMM-Newton telescopes and the more uniform point spread function (PSF) throughout the EPIC field of view result in a higher efficiency of the EPIC camera in detecting off-axis sources (thus in a larger sky area) than ACIS and HRC detectors. Chandra has a higher spatial resolution near the center of the field of view, but its PSF profile rapidly degrades at increasing off-axis angles thus reducing the efficiency in detecting faint sources.

The Sun activity is characterized by the 11-yr cycle. The maximum variation of amplitudes in the Sun cycle is a factor greater than 20 . In order to study the time variability on time scales of about 12 years we have compared the X-ray luminosities of NGC 2516 stars falling in the field of view of ROSAT observations taken in 1993 and analyzed by Jeffries et al. (1997). This time scale should allow us to detect stellar cycles present in NGC 2516 stars with amplitudes and periods similar to those observed on the Sun. Figure 10 shows the X-ray luminosities measured with XMM-Newton and with ROSAT; XMM-Newton luminosities were recalculated coherently in the ROSAT band. Error bars for both XMM and ROSAT $L_{\mathrm{X}}$ are plotted, the latter obtained from Jeffries et al. (1997). The lowest $L_{\mathrm{X}}$ detected with ROSAT is $\sim 10^{29} \mathrm{erg} / \mathrm{s}$. Only two stars detected in both surveys, JTH 9465 (also JTX 138 in SIMBAD) and JTH 10817 (JTX 114), changed luminosity by a factor between 2 and 5, due to flare variability during one of the six XMM-Newton observations. The variability of these two stars is discussed in Ramsay et al. (2003). Another four stars (JTH 10863, 10871, 12649 and 15514), undetected by ROSAT, are interesting because they are variable in the XMM survey by more than a factor of two with respect to their ROSAT upper limits. Most of the stars have variations within a factor of two. The modest variability on long time scales has been 


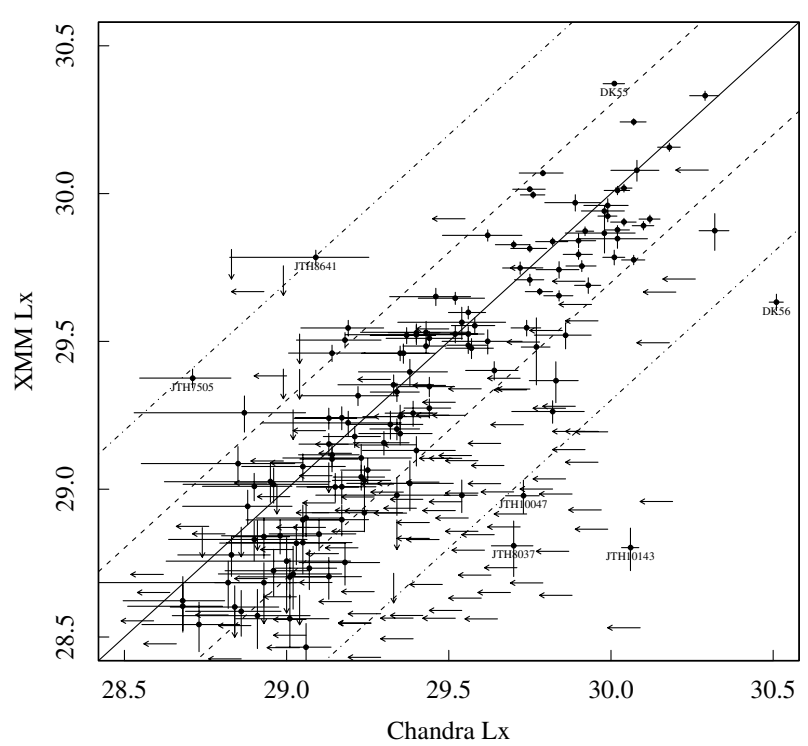

Fig. 9. XMM-Newton vs. Chandra (Damiani et al. 2003) measurements of X-ray luminosities of NGC 2516 stars detected at least once in the two surveys. Error bars are the statistical uncertainties; the horizontal arrows mark upper limits for undetected stars in Chandra, while vertical ones refer to XMM-Newton undetected stars. Solid, dashed and dot-dashed lines trace the equal, two and five times (and their reciprocal) ratios. We indicate the names of a few stars with large variability, JTH are from Jeffries et al. (2001), while DK refers to Dachs \& Kabus (1989).

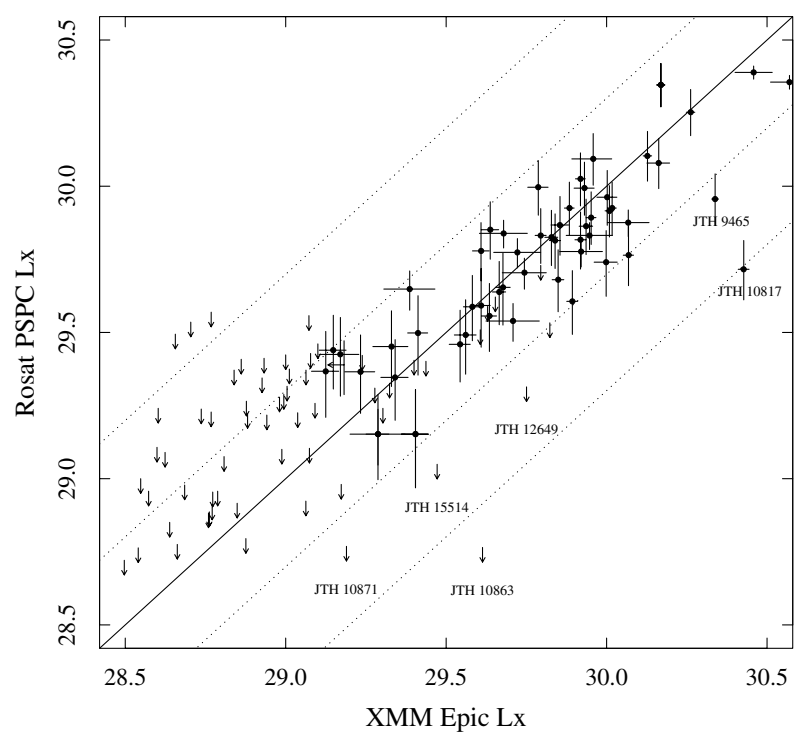

Fig. 10. X-ray luminosities of XMM detected stars of NGC 2516 compared with values obtained with ROSAT satellite by Jeffries et al. (1997). Arrows mark upper limits due to ROSAT undetected stars. The continuous line and dotted lines correspond to 1, 1/5,1/2, 2 and 5 ratio between XMM-Newton and ROSAT luminosities, respectively.

reported by Wolk et al. (2004), in a study of X-ray time variability of NGC 2516 based on Chandra data. These variations are smaller than expected in the presence of solar cycles and strongly suggest that cycles in young stars are absent or have different periods and/or smaller amplitudes than those observed in the Sun.

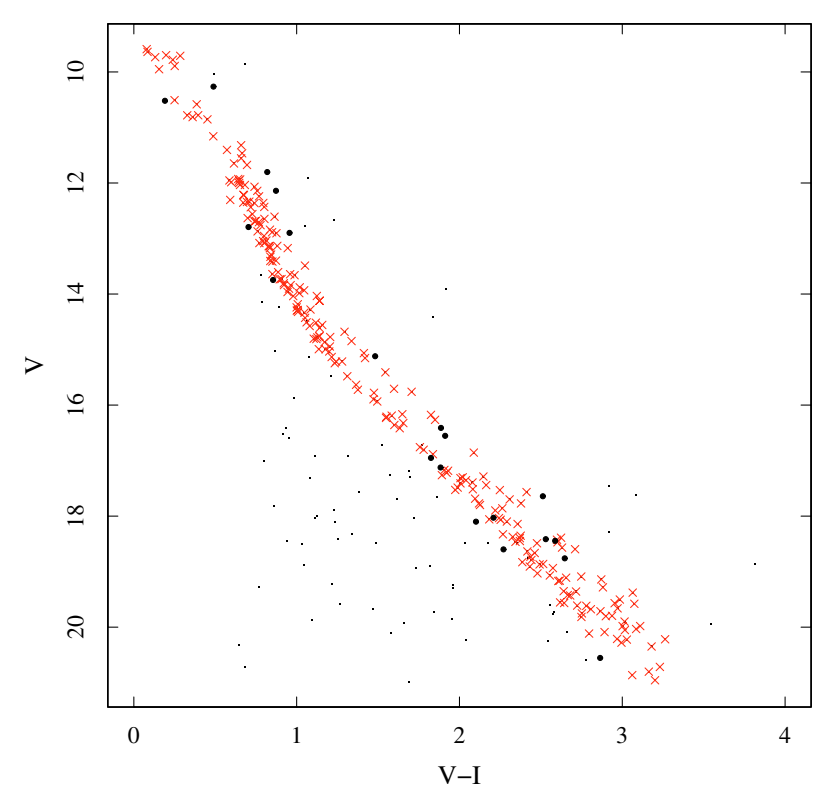

Fig. 11. Color-magnitude diagram of X-ray sources matching the optical catalog of Jeffries et al. (2001). Small dots: all sources, crosses: NGC 2516 stars, large dots: suggested new cluster members.

\section{New possible members and unidentified sources}

A number of X-ray detections have optical photometry consistent with the membership of NGC 2516. On this basis these stars could be cluster members. Figure 11 shows the colormagnitude diagram of the X-ray sources from the Jeffries catalog. By placing them at the cluster distance, their X-ray luminosities range between $3.7 \times 10^{28}$ and $1.5 \times 10^{30} \mathrm{erg} / \mathrm{s}$. In Table 4 we list the main properties of these objects.

A few X-ray sources do not have counterparts in optical or infrared bands. After having searched for optical counterparts of the X-ray sources in GSC-II, 2MASS and DENIS and the complete catalog of Jeffries et al. (2001), 49 sources are left without optical/infrared known counterparts. We have marked these sources with a $U$ in the last column of Table A.1: the finding charts at their positions are reported in Figs. D.1 and D.2.

\section{Summary}

We have presented results from a deep X-ray survey of the young open cluster NGC 2516, obtained from a series of six XMM-Newton observations. The source detection has been carried out on the sum of EPIC MOS and pn images with a wavelet transform code (PWXDetect). We have reached fluxes lower by a factor of 5 or more than Chandra observations carried out in the same epoch. We have detected 431 sources, 234 of them have as optical counterparts 239 cluster members, five X-ray sources match as many close pairs. X-ray emission of spectral type from early B down to M-type stars has been detected; the coolest detected stars have spectral type M5 and $T_{\text {eff }} \sim 3000$. We have investigated the X-ray spectral properties of cluster stars through 1-T and 2-T models to estimate the dominant plasma thermal components and emission measures. For 1-T models we find temperatures around 
Table 4. Properties of new suggested cluster members. Columns are: id. number in Jeffries et al. (2001) catalog, X-ray source number as in Table A.1, $V, B-V, V-I$, match distance between $\mathrm{X}$ and optical position, flux and $\mathrm{X}$-ray luminosity in $0.3-7.9 \mathrm{keV}$, calculated by assuming them as cluster members.

\begin{tabular}{llllllll}
\hline \hline $\begin{array}{l}\text { Opt id } \\
\text { JTH\# }\end{array}$ & X Src & $\begin{array}{l}V \\
\text { mag }\end{array}$ & $\begin{array}{l}B-V \\
\mathrm{mag}\end{array}$ & $\begin{array}{l}V-I \\
\mathrm{mag}\end{array}$ & $\begin{array}{l}r \\
\prime\end{array}$ & $\begin{array}{l}\log f_{\mathrm{X}} \\
\mathrm{erg} \mathrm{s}^{-1} \mathrm{~cm}^{-2}\end{array}$ & $\begin{array}{l}\log L_{\mathrm{X}} \\
\mathrm{erg} \mathrm{s}^{-1}\end{array}$ \\
\hline 5337 & 22 & 16.412 & 1.288 & 1.887 & 0.8 & -13.44 & 29.81 \\
7417 & 53 & 18.418 & 1.276 & 2.53 & 0.9 & -13.96 & 29.29 \\
6220 & 67 & 15.123 & 1.177 & 1.481 & 0.9 & -13.39 & 29.87 \\
9976 & 72 & 17.123 & 1.312 & 1.884 & 0.6 & -14.04 & 29.21 \\
7485 & 75 & 11.801 & 0.771 & 0.819 & 1.1 & -13.97 & 29.28 \\
9022 & 76 & 18.448 & 1.74 & 2.587 & 1.6 & -14.08 & 29.18 \\
8692 & 88 & 18.029 & 1.379 & 2.208 & 0.8 & -14.68 & 28.57 \\
7200 & 93 & 18.599 & - & 2.27 & 2.2 & -14.48 & 28.78 \\
7708 & 110 & 17.643 & 1.714 & 2.512 & 2.8 & -13.84 & 29.41 \\
5447 & 167 & 16.555 & 1.348 & 1.912 & 1.5 & -13.76 & 29.5 \\
7129 & 170 & 18.098 & - & 2.101 & 0.2 & -14.06 & 29.19 \\
7147 & 217 & 13.747 & 0.726 & 0.856 & 0.7 & -13.55 & 29.7 \\
9492 & 243 & 10.518 & 0.148 & 0.191 & 1.6 & -14.69 & 28.57 \\
11455 & 264 & 18.759 & 1.348 & 2.646 & 1.5 & -14.64 & 28.61 \\
11366 & 292 & 12.898 & 0.827 & 0.956 & 2.2 & -13.07 & 30.18 \\
7844 & 324 & 12.14 & 0.368 & 0.873 & 2 & -14.05 & 29.21 \\
9106 & 341 & 10.262 & 0.405 & 0.49 & 1.8 & -14.49 & 28.76 \\
9467 & 348 & 16.95 & 1.132 & 1.825 & 0.3 & -14.21 & 29.04 \\
4181 & 352 & 20.553 & - & 2.865 & 2.6 & -13.45 & 29.8 \\
6700 & 413 & 12.792 & 0.597 & 0.704 & 2.2 & -14.08 & 29.17 \\
\hline
\end{tabular}

$0.6 \mathrm{keV}$. In 2-T models the temperatures are in $0.3-0.7 \mathrm{keV}$ and $1.0-2.0 \mathrm{keV}$ ranges in agreement with the temperatures found in nearly coeval open clusters like Pleiades and Blanco 1.

For each spectral type F, G, K, M we estimated the maximum likelihood distribution of X-ray luminosities taking also into account the upper limits for undetected cluster stars. G-, $\mathrm{K}-$, and M- type stars are under-luminous with respect to the Pleiades in the same spectral range. Possible biases due to contaminating field stars are discussed and statistical corrections have been made to X-ray distribution functions. We conclude that the NGC 2516 solar type stars are definitively less luminous in X-rays than the analog Pleiades. The differences could be attributed mainly to the slight older age of NGC 2516 (140 Myr vs. 80-100 Myr of Pleiades), less probably to a lower rotation rate. The past cluster history could also have a role in determining X-ray emission.

By comparing XMM-EPIC and ROSAT-PSPC data we explored X-ray variability on time scales comparable to the solar cycle. We observe only variations in X-ray luminosities by a factor of 2-3. Along with what is seen in other coeval open clusters, this result strongly suggests that activity cycles of amplitude and periods like the solar one are not present in young Main Sequence stars at an age of 140 Myr.

We identify 20 likely new candidate members on the basis of their X-ray emission and optical photometry. 49 X-ray sources are left unidentified in optical and infrared bands and are likely of extragalactic nature and for them we provide finding charts.
Acknowledgements. Authors acknowledge financial support from MIUR-PR/N grant (Ministero dell'Istruzione, Università e Ricerca).

\section{References}

Babel, J., \& Montmerle, T. 1997, ApJ, 485, L29

Briggs, K. R., \& Pye, J. P. 2003, MNRAS, 345, 714

Cassinelli, J. P., \& MacGregor, K. B. 2000, in ASP Conf. Ser., 337

Cox, A. N. 2000, Allen's astrophysical quantities, Allen's astrophysical quantities, ed. A. N. Cox, 4th ed. (New York: AIP Press; Springer)

Dachs, J. 1970, A\&A, 5, 312

Dachs, J., \& Kabus, H. 1989, A\&AS, 78, 25

Damiani, F., Flaccomio, E., Micela, G., et al. 2003, ApJ, 588, 1009

Damiani, F., Maggio, A., Micela, G., \& Sciortino, S. 1997a, ApJ, 483, 350

Damiani, F., Maggio, A., Micela, G., \& Sciortino, S. 1997b, ApJ, 483, 370

Favata, F., \& Micela, G. 2003, Space Sci. Rev., 108, 577

Feigelson, E. D., \& Nelson, P. I. 1985, ApJ, 293, 192

Feldmeier, A. 1995, A\&A, 299, 523

Gondoin, P. 2005, A\&A, 438, 291

Güdel, M. 2004, A\&AR, 12, 71

Harnden, F. R., Adams, N. R., Damiani, F., et al. 2001, ApJ, 547, L141

Jeffries, R. D., James, D. J., \& Thurston, M. R. 1998, MNRAS, 300, 550

Jeffries, R. D., Thurston, M. R., \& Hambly, N. C. 2001, A\&A, 375, 863

Jeffries, R. D., Thurston, M. R., \& Pye, J. P. 1997, MNRAS, 287, 350

Kenyon, S. J., \& Hartmann, L. 1995, ApJS, 101, 117

Lucy, L. B., \& White, R. L. 1980, ApJ, 241, 300 
Marino, A., Micela, G., Peres, G., Pillitteri, I., \& Sciortino, S. 2004, $\mathrm{A} \& \mathrm{~A}$, in press

Meynet, G., Mermilliod, J.-C., \& Maeder, A. 1993, A\&AS, 98, 477

Micela, G., Sciortino, S., Harnden, et al. 1999, A\&A, 341, 751

Micela, G., Sciortino, S., Jeffries, R. D., Thurston, M. R., \& Favata, F. 2000, A\&A, 357, 909

Pallavicini, R., Golub, L., Rosner, R., et al. 1981, ApJ, 248, 279

Patten, B. M., \& Simon, T. 1996, ApJS, 106, 489

Pillitteri, I., Micela, G., Sciortino, S., Damiani, F., \& Harnden, F. R. 2004, A\&A, 421, 175

Pizzolato, N., Maggio, A., Micela, G., Sciortino, S., \& Ventura, P. 2003, A\&A, 397, 147

Prosser, C. F., Stauffer, J. R., Caillault, J.-P., et al. 1995, AJ, 110, 1229

Ramsay, G., Harra, L., \& Kay, H. 2003, MNRAS, 341, 1388

Randich, S. 2000, in Stellar Clusters and Associations: Convection, Rotation, and Dynamos, ASP Conf. Ser., 198, 401
Rauw, G., De Becker, M., Gosset, E., Pittard, J. M., \& Stevens, I. R. 2003, A\&A, 407, 925

Schmitt, J. H. M. M. 1997, A\&A, 318, 215

Schmitt, J. H. M. M., Fleming, T. A., \& Giampapa, M. S. 1995, ApJ, 450,392

Sciortino, S., Micela, G., Damiani, F., et al. 2001, A\&A, 365, L259

Siess, L., Dufour, E., \& Forestini, M. 2000, A\&A, 358, 593

Terndrup, D. M., Pinsonneault, M., Jeffries, R. D., et al. 2002, ApJ, 576,950

Vaiana, G. S., Fabbiano, G., Giacconi, R., et al. 1981, ApJ, 245, 163

van den Berg, M., Tagliaferri, G., Belloni, T., \& Verbunt, F. 2004, A\&A, 418, 509

Waldron, W. L., \& Cassinelli, J. P. 2001, ApJ, 548, L45

Wolk, S. J., Harnden, F. R., Murray, S. S., et al. 2004, ApJ, 606, 466 
I. Pillitteri et al.: Deep X-ray survey of NGC 2516 with XMM-Newton, Online Material p 1

\section{Online Material}


I. Pillitteri et al.: Deep X-ray survey of NGC 2516 with XMM-Newton, Online Material p 2

Appendix A: X-ray detections 


\section{Pillitteri et al.: Deep X-ray survey of NGC 2516 with XMM-Newton, Online Material p 3}

Table A.1. List of detected X-ray sources in the combined images of the XMM-Newton EPIC camera. Columns report: running number of $\mathrm{X}$-ray detection, coordinates, count rate in MOS1 equivalent counts, detection significance (in units of background mean standard deviation), total exposure time, offaxis distance and unidentified source flag (cf. Sect. 4).

\begin{tabular}{|c|c|c|c|c|c|c|c|}
\hline$\overline{\text { Num }}$ & $\begin{array}{l}\text { RA } \\
\text { J2000 }\end{array}$ & $\begin{array}{l}\text { Dec } \\
\text { J2000 }\end{array}$ & $\begin{array}{l}\text { Count rate } \\
\text { cts ks }^{-1}\end{array}$ & $\bar{~} \overline{\text { Signif. }}$ & $\begin{array}{l}\text { Exp. time } \\
\text { ks }\end{array}$ & $\begin{array}{l}\text { Offaxis } \\
\text { arcmin }\end{array}$ & Un. \\
\hline 1 & $07: 57: 20.63$ & $-61: 4: 19.27$ & $4.38 \pm 0.82$ & 10.74 & 8 & 18.46 & \\
\hline 4 & $07: 58: 23.5$ & $-61: 3: 44.57$ & $1.49 \pm 0.27$ & 7.59 & 49.55 & 16.56 & \\
\hline 6 & 07:57:17.67 & $-61: 3: 30.6$ & $7.05 \pm 0.75$ & 14.34 & 16.66 & 17.85 & \\
\hline 7 & 07:57:40.35 & $-61: 3: 31$ & $0.62 \pm 0.16$ & 5.81 & 46.76 & 16.93 & \\
\hline 8 & 07:58:34.76 & $-61: 3: 17.86$ & $1.93 \pm 0.29$ & 9.28 & 50.52 & 16.23 & \\
\hline 9 & $07: 57: 47.6$ & $-61: 3: 6.98$ & $0.84 \pm 0.17$ & 7.21 & 50.04 & 16.32 & \\
\hline 10 & 07:58:26.91 & $-61: 2: 46.14$ & $1.16 \pm 0.21$ & 8.07 & 54.34 & 15.61 & \\
\hline 11 & 07:58:44.52 & $-61: 2: 37.93$ & $0.68 \pm 0.2$ & 5.66 & 28.18 & 15.78 & \\
\hline 12 & $07: 57: 38.58$ & $-61: 2: 10.9$ & $4.83 \pm 0.4$ & 21.14 & 49.11 & 15.71 & \\
\hline 13 & $07: 58: 12.85$ & $-61: 2: 10.28$ & $2.82 \pm 0.3$ & 14.41 & 57.71 & 14.98 & $\mathrm{U}$ \\
\hline 15 & 07:57:35.79 & $-61: 2: 6.54$ & $0.73 \pm 0.2$ & 6.16 & 29.76 & 15.74 & \\
\hline 16 & 07:57:57.76 & $-61: 2: 5.06$ & $1.04 \pm 0.19$ & 7.2 & 56.76 & 15.07 & \\
\hline 18 & 07:58:43.13 & $-61: 1: 59.66$ & $2.51 \pm 0.29$ & 14.52 & 49.01 & 15.12 & $\mathrm{U}$ \\
\hline 19 & 07:59:28.83 & $-61: 1: 58.01$ & $1.06 \pm 0.24$ & 6.21 & 38.19 & 17.13 & \\
\hline 20 & 07:57:10.68 & $-61: 1: 27.34$ & $1.22 \pm 0.27$ & 5.47 & 46.06 & 16.4 & \\
\hline 21 & 07:57:56.85 & $-61: 0: 58.75$ & $1.73 \pm 0.2$ & 13.38 & 68.94 & 14 & \\
\hline 22 & $07: 56: 56.25$ & $-61: 0: 57.17$ & $3.79 \pm 0.77$ & 7.24 & 10.86 & 16.93 & \\
\hline 23 & 07:59:36.78 & $-61: 0: 57.02$ & $1.22 \pm 0.24$ & 7.13 & 41.21 & 16.8 & $\mathrm{U}$ \\
\hline 24 & 07:58:53.92 & $-61: 0: 24.48$ & $0.9 \pm 0.15$ & 8.55 & 75.47 & 13.93 & \\
\hline 25 & 07:57:43.44 & $-61: 0: 0.79$ & $0.99 \pm 0.16$ & 8.38 & 68.58 & 13.46 & \\
\hline 27 & 07:58:3.12 & $-60: 59: 56.29$ & $1.62 \pm 0.18$ & 15.07 & 85.46 & 12.85 & \\
\hline 29 & $07: 58: 32.24$ & $-60: 59: 48.62$ & $1.07 \pm 0.15$ & 10.28 & 108.33 & 12.74 & \\
\hline 30 & $07: 57: 8.39$ & $-60: 59: 45.06$ & $1.24 \pm 0.24$ & 6.62 & 51.24 & 15.1 & \\
\hline 31 & $07: 58: 9.17$ & $-60: 59: 42.36$ & $0.39 \pm 0.08$ & 6.72 & 111.72 & 12.54 & \\
\hline 32 & 07:57:31.49 & $-60: 59: 30.8$ & $4.87 \pm 0.35$ & 28.3 & 61.47 & 13.52 & \\
\hline 34 & $07: 58: 22.4$ & $-60: 59: 9.02$ & $0.31 \pm 0.06$ & 6.56 & 121.29 & 11.96 & \\
\hline 35 & 07:58:35.72 & $-60: 59: 2.62$ & $0.55 \pm 0.1$ & 7.84 & 133.7 & 12.05 & $\mathrm{U}$ \\
\hline 36 & $07: 56: 38.76$ & $-60: 58: 58.84$ & $1.84 \pm 0.39$ & 6.36 & 16.43 & 16.82 & \\
\hline 37 & $07: 57: 7.52$ & $-60: 58: 55.7$ & $0.66 \pm 0.17$ & 6.19 & 43.66 & 14.48 & $\mathrm{U}$ \\
\hline 38 & 07:58:21.47 & $-60: 58: 52.1$ & $1.46 \pm 0.15$ & 13.52 & 139.39 & 11.68 & \\
\hline 39 & 07:59:32.14 & $-60: 58: 47.89$ & $2.16 \pm 0.27$ & 12.41 & 50.32 & 14.73 & \\
\hline 40 & 07:58:11.66 & $-60: 58: 38.14$ & $0.74 \pm 0.1$ & 10.85 & 146.54 & 11.46 & $\mathrm{U}$ \\
\hline 41 & 07:58:6.65 & $-60: 58: 34.18$ & $0.38 \pm 0.08$ & 6.2 & 146.23 & 11.44 & \\
\hline 42 & 07:59:25.06 & $-60: 58: 30.29$ & $5.36 \pm 0.36$ & 26 & 69.39 & 13.98 & $\mathrm{U}$ \\
\hline 43 & $07: 58: 31.7$ & $-60: 58: 28.13$ & $0.41 \pm 0.09$ & 6.17 & 138.94 & 11.4 & \\
\hline 44 & $07: 57: 53.41$ & $-60: 58: 22.01$ & $4.82 \pm 0.24$ & 35.53 & 133 & 11.54 & \\
\hline 45 & 07:58:51.45 & $-60: 58: 15.78$ & $0.69 \pm 0.1$ & 9.58 & 135.04 & 11.81 & \\
\hline 46 & $07: 56: 55$ & $-60: 58: 13.76$ & $1.12 \pm 0.2$ & 9.38 & 49.13 & 14.91 & \\
\hline 47 & $07: 57: 27.7$ & $-60: 58: 13.51$ & $1.02 \pm 0.21$ & 7.05 & 59.56 & 12.57 & \\
\hline 48 & $07: 59: 5.46$ & $-60: 58: 9.3$ & $0.75 \pm 0.11$ & 9.42 & 118.15 & 12.42 & \\
\hline 49 & $07: 57: 21.21$ & $-60: 58: 4.08$ & $0.81 \pm 0.16$ & 6.71 & 65.03 & 12.84 & $\mathrm{U}$ \\
\hline 50 & 07:59:32.98 & $-60: 57: 58.25$ & $1.03 \pm 0.18$ & 8.2 & 66.45 & 14.16 & $\mathrm{U}$ \\
\hline 51 & 07:58:20.05 & $-60: 57: 54.54$ & $0.65 \pm 0.1$ & 9.52 & 134.22 & 10.71 & $\mathrm{U}$ \\
\hline 53 & 07:57:51.12 & $-60: 57: 29.59$ & $1.15 \pm 0.12$ & 12.6 & 152.69 & 10.78 & \\
\hline 55 & $07: 56: 34.67$ & $-60: 57: 25.31$ & $1.79 \pm 0.33$ & 10.19 & 17.21 & 16.15 & \\
\hline 56 & $07: 57: 31.24$ & $-60: 57: 27.14$ & $0.72 \pm 0.14$ & 6.58 & 108.83 & 11.69 & $\mathrm{U}$ \\
\hline 57 & 07:58:52.02 & $-60: 57: 26.57$ & $0.75 \pm 0.1$ & 11.02 & 134.6 & 11.07 & \\
\hline 58 & $07: 57: 7.35$ & $-60: 57: 5.22$ & $1.16 \pm 0.2$ & 7.24 & 65.92 & 13.06 & \\
\hline 59 & 07:59:8.48 & $-60: 57: 3.49$ & $0.64 \pm 0.11$ & 9.36 & 139.69 & 11.65 & \\
\hline 60 & 07:57:59.19 & $-60: 56: 53.95$ & $5.67 \pm 0.21$ & 55.7 & 177.01 & 9.95 & \\
\hline 61 & $07: 57: 25.25$ & $-60: 56: 50.57$ & $1.85 \pm 0.18$ & 14.23 & 83.85 & 11.54 & \\
\hline 62 & 07:58:49.72 & $-60: 56: 50.86$ & $0.29 \pm 0.06$ & 6.48 & 176.33 & 10.42 & $\mathrm{U}$ \\
\hline 63 & 07:56:47.55 & $-60: 56: 48.3$ & $7.04 \pm 0.57$ & 20.41 & 31.91 & 14.56 & \\
\hline 64 & $07: 59: 9.25$ & $-60: 56: 39.91$ & $1.02 \pm 0.12$ & 12.65 & 144.98 & 11.37 & \\
\hline 65 & 07:58:53.66 & $-60: 56: 34.04$ & $0.97 \pm 0.1$ & 15.55 & 179.67 & 10.35 & \\
\hline 66 & 07:58:58.09 & $-60: 56: 33.22$ & $0.36 \pm 0.07$ & 6.57 & 177.39 & 10.58 & \\
\hline
\end{tabular}


Table A.1. continued.

\begin{tabular}{|c|c|c|c|c|c|c|c|}
\hline Num & $\begin{array}{l}\text { RA } \\
\text { J2000 }\end{array}$ & $\begin{array}{l}\text { Dec } \\
\text { J2000 }\end{array}$ & $\begin{array}{l}\text { Count rate } \\
\text { cts ks }{ }^{-1}\end{array}$ & Signif. & $\begin{array}{l}\text { Exp. time } \\
\text { ks }\end{array}$ & $\begin{array}{l}\text { Offaxis } \\
\text { arcmin }\end{array}$ & Un. \\
\hline 67 & $07: 57: 19.36$ & $-60: 56: 29.11$ & $4.32 \pm 0.23$ & 35.57 & 119.41 & 11.67 & \\
\hline 68 & $07: 57: 45.01$ & $-60: 56: 20.29$ & $0.3 \pm 0.07$ & 6.11 & 179.71 & 9.95 & \\
\hline 69 & $07: 57: 47.54$ & $-60: 56: 19.28$ & $0.72 \pm 0.1$ & 9.08 & 176.81 & 9.82 & \\
\hline 70 & $07: 57: 30.2$ & $-60: 56: 16.01$ & $0.4 \pm 0.08$ & 6.28 & 144.77 & 10.73 & \\
\hline 71 & $07: 58: 50.67$ & $-60: 56: 3.77$ & $1.15 \pm 0.1$ & 17.04 & 191.13 & 9.74 & $\mathrm{U}$ \\
\hline 72 & $07: 58: 55.42$ & $-60: 55: 59.63$ & $0.95 \pm 0.1$ & 14.62 & 179.62 & 9.93 & \\
\hline 73 & $07: 59: 34.23$ & $-60: 55: 53.22$ & $2.21 \pm 0.18$ & 16.91 & 118.09 & 12.76 & \\
\hline 74 & $07: 57: 26.72$ & $-60: 55: 49.8$ & $0.32 \pm 0.07$ & 5.58 & 165.11 & 10.61 & \\
\hline 75 & $07: 57: 52.91$ & $-60: 55: 45.12$ & $1.12 \pm 0.09$ & 18.95 & 200.44 & 9.06 & \\
\hline 76 & $07: 58: 32.68$ & $-60: 55: 44.33$ & $0.88 \pm 0.1$ & 12.42 & 210.36 & 8.74 & \\
\hline 77 & $07: 57: 20.65$ & $-60: 55: 43.07$ & $1.39 \pm 0.13$ & 14.44 & 158.56 & 10.97 & \\
\hline 78 & $07: 58: 48.6$ & $-60: 55: 25.93$ & $0.61 \pm 0.08$ & 10.86 & 202.74 & 9.06 & \\
\hline 79 & $07: 58: 43.22$ & $-60: 55: 25.82$ & $10.55 \pm 0.26$ & 94.66 & 208.29 & 8.81 & \\
\hline 80 & $07: 59: 23.15$ & $-60: 55: 13.22$ & $0.33 \pm 0.06$ & 7.52 & 160.1 & 11.33 & \\
\hline 81 & $07: 58: 18.23$ & $-60: 55: 10.06$ & $0.3 \pm 0.05$ & 8.56 & 215.57 & 7.97 & \\
\hline 84 & $07: 57: 56.18$ & $-60: 54: 58.57$ & $1.32 \pm 0.11$ & 18.02 & 212.38 & 8.19 & \\
\hline 85 & $07: 58: 23.35$ & $-60: 54: 58.18$ & $7.15 \pm 0.2$ & 78.44 & 225.84 & 7.8 & \\
\hline 86 & $07: 57: 57.2$ & $-60: 54: 56.02$ & $0.18 \pm 0.04$ & 8.38 & 215.99 & 8.11 & \\
\hline 87 & $07: 58: 16.68$ & $-60: 54: 52.56$ & $0.28 \pm 0.05$ & 8.36 & 226.1 & 7.67 & \\
\hline 88 & $07: 58: 24.46$ & $-60: 54: 40.68$ & $0.22 \pm 0.05$ & 8.26 & 229.69 & 7.53 & \\
\hline 89 & $07: 57: 37.8$ & $-60: 54: 33.19$ & $2.55 \pm 0.14$ & 33.85 & 198.47 & 8.79 & \\
\hline 90 & $07: 58: 53.58$ & $-60: 54: 25.74$ & $1.48 \pm 0.11$ & 23.87 & 210.34 & 8.46 & \\
\hline 91 & $07: 58: 27.26$ & $-60: 54: 24.84$ & $0.2 \pm 0.05$ & 5.07 & 216.35 & 7.31 & $\mathrm{U}$ \\
\hline 92 & 07:57:54.42 & $-60: 54: 22.75$ & $0.18 \pm 0.04$ & 5.69 & 221.64 & 7.7 & \\
\hline 93 & $07: 57: 45.08$ & $-60: 54: 18$ & $0.35 \pm 0.06$ & 7.25 & 209.26 & 8.12 & \\
\hline 94 & $07: 58: 47.85$ & $-60: 54: 15.62$ & $6.5 \pm 0.2$ & 69.17 & 220.94 & 7.97 & \\
\hline 95 & $07: 58: 18.51$ & $-60: 54: 15.77$ & $0.26 \pm 0.05$ & 6.52 & 230.77 & 7.06 & \\
\hline 96 & $07: 56: 47.45$ & $-60: 54: 12.06$ & $2 \pm 0.22$ & 16.19 & 65.93 & 13 & \\
\hline 97 & $08: 00: 2.25$ & $-60: 53: 54.31$ & $1.28 \pm 0.2$ & 11.02 & 56.36 & 14.42 & \\
\hline 98 & $07: 57: 24.59$ & $-60: 53: 54.42$ & $0.21 \pm 0.05$ & 5.38 & 183.08 & 9.29 & \\
\hline 99 & $07: 58: 31.93$ & $-60: 53: 52.87$ & $1.22 \pm 0.09$ & 22.46 & 240.29 & 6.91 & \\
\hline 100 & $07: 56: 59.9$ & $-60: 53: 47.76$ & $0.77 \pm 0.11$ & 9.57 & 127.24 & 11.52 & \\
\hline 102 & $07: 57: 21.46$ & $-60: 53: 43.48$ & $0.57 \pm 0.1$ & 7.29 & 160.85 & 9.43 & \\
\hline 103 & 07:57:57.67 & $-60: 53: 40.52$ & $4.02 \pm 0.16$ & 45.13 & 227.04 & 6.91 & \\
\hline 104 & 07:58:52.29 & $-60: 53: 38.83$ & $2.3 \pm 0.12$ & 35.31 & 222.43 & 7.72 & \\
\hline 105 & $07: 58: 31.57$ & $-60: 53: 11.65$ & $2.09 \pm 0.11$ & 36.48 & 247.59 & 6.24 & \\
\hline 106 & $07: 57: 47$ & $-60: 53: 9.02$ & $0.51 \pm 0.08$ & 8.09 & 226.47 & 7.01 & $\mathrm{U}$ \\
\hline 107 & $07: 57: 15.49$ & $-60: 53: 2.47$ & $0.47 \pm 0.08$ & 8.17 & 165.29 & 9.54 & \\
\hline 108 & $07: 57: 55.2$ & $-60: 52: 58.66$ & $0.18 \pm 0.04$ & 7.15 & 241.77 & 6.38 & \\
\hline 109 & 07:58:41.92 & $-60: 52: 55.81$ & $0.53 \pm 0.08$ & 7.62 & 224.36 & 6.46 & \\
\hline 110 & 07:57:57.96 & $-60: 52: 48.72$ & $1.52 \pm 0.1$ & 25.24 & 220.39 & 6.09 & \\
\hline 111 & $07: 58: 4.58$ & $-60: 52: 44.22$ & $0.65 \pm 0.07$ & 14.01 & 249.66 & 5.75 & \\
\hline 112 & 08:00:13.26 & $-60: 52: 40.44$ & $2.85 \pm 0.35$ & 12.23 & 50.02 & 15.14 & \\
\hline 113 & 07:59:3.77 & $-60: 52: 35.58$ & $1.57 \pm 0.11$ & 27.54 & 209.1 & 7.81 & $\mathrm{U}$ \\
\hline 114 & $07: 57: 41.17$ & $-60: 52: 31.66$ & $0.78 \pm 0.09$ & 11.82 & 219 & 6.92 & \\
\hline 115 & $07: 57: 30.37$ & $-60: 52: 29.68$ & $0.21 \pm 0.05$ & 6.76 & 182.97 & 7.8 & \\
\hline 116 & $07: 58: 31.33$ & $-60: 52: 25.64$ & $0.25 \pm 0.04$ & 8.64 & 239.62 & 5.49 & \\
\hline 117 & 07:59:20.41 & $-60: 52: 22.37$ & $0.64 \pm 0.09$ & 8.49 & 183.63 & 9.26 & \\
\hline 118 & $07: 58: 48.46$ & $-60: 52: 21.83$ & $2.79 \pm 0.13$ & 36.22 & 244.64 & 6.4 & \\
\hline 119 & $07: 59: 56.3$ & $-60: 52: 17.83$ & $0.83 \pm 0.13$ & 8.86 & 104.87 & 13.08 & \\
\hline 120 & $07: 58: 27.54$ & $-60: 52: 19.13$ & $0.53 \pm 0.06$ & 12.82 & 268.81 & 5.26 & \\
\hline 121 & $07: 58: 13.86$ & $-60: 52: 17.62$ & $0.27 \pm 0.05$ & 9.49 & 263.72 & 5.11 & \\
\hline 122 & 07:58:56.57 & $-60: 52: 15.49$ & $0.98 \pm 0.09$ & 15.88 & 224.55 & 6.95 & \\
\hline 123 & $07: 57: 18.27$ & $-60: 52: 12.83$ & $0.32 \pm 0.06$ & 7 & 147.54 & 8.78 & $\mathrm{U}$ \\
\hline 124 & $07: 57: 29.35$ & $-60: 52: 11.64$ & $4.52 \pm 0.21$ & 41.44 & 145.8 & 7.7 & $\mathrm{U}$ \\
\hline 125 & 07:58:16.66 & $-60: 52: 12.11$ & $0.3 \pm 0.05$ & 10.32 & 272.54 & 5 & \\
\hline 126 & 07:58:0.31 & $-60: 52: 12.04$ & $2.67 \pm 0.12$ & 44.93 & 253.55 & 5.42 & \\
\hline 127 & 07:59:15.24 & $-60: 52: 5.23$ & $0.37 \pm 0.06$ & 10.64 & 201.76 & 8.57 & \\
\hline 128 & $07: 57: 38.65$ & $-60: 52: 3.25$ & $0.36 \pm 0.06$ & 7.56 & 170.89 & 6.77 & \\
\hline
\end{tabular}


I. Pillitteri et al.: Deep X-ray survey of NGC 2516 with XMM-Newton, Online Material p 5

Table A.1. continued.

\begin{tabular}{|c|c|c|c|c|c|c|c|}
\hline$\overline{\text { Num }}$ & $\begin{array}{l}\text { RA } \\
\text { J2000 }\end{array}$ & $\begin{array}{l}\text { Dec } \\
\text { J2000 }\end{array}$ & $\begin{array}{l}\text { Count rate } \\
\mathrm{cts} \mathrm{ks}^{-1}\end{array}$ & Signif. & $\begin{array}{l}\text { Exp. time } \\
\text { ks }\end{array}$ & $\begin{array}{l}\text { Offaxis } \\
\text { arcmin }\end{array}$ & Un. \\
\hline 129 & $07: 59: 6.91$ & $-60: 52: 1.81$ & $0.85 \pm 0.08$ & 17.61 & 201.75 & 7.73 & \\
\hline 130 & 07:59:59.25 & $-60: 51: 59.47$ & $0.17 \pm 0.05$ & 5.21 & 105.55 & 13.3 & $\mathrm{U}$ \\
\hline 131 & $07: 58: 19.33$ & $-60: 52: 0.08$ & $0.47 \pm 0.06$ & 10.55 & 261.88 & 4.81 & \\
\hline 132 & 07:58:10.58 & $-60: 51: 57.53$ & $2.68 \pm 0.11$ & 43.78 & 268.69 & 4.83 & \\
\hline 133 & 07:59:17.95 & $-60: 51: 49.28$ & $0.12 \pm 0.03$ & 6 & 195.26 & 8.7 & $\mathrm{U}$ \\
\hline 135 & $07: 57: 2.9$ & $-60: 51: 43.16$ & $0.42 \pm 0.08$ & 7.16 & 161.57 & 10.14 & \\
\hline 136 & 08:00:23.66 & $-60: 51: 40.36$ & $1.38 \pm 0.32$ & 5.69 & 35.34 & 16.02 & $\mathrm{U}$ \\
\hline 137 & $07: 56: 22.68$ & $-60: 51: 38.88$ & $4.4 \pm 0.63$ & 12.03 & 20.12 & 14.67 & \\
\hline 138 & $07: 56: 41.56$ & $-60: 51: 37.91$ & $1.13 \pm 0.17$ & 9.43 & 66.07 & 12.49 & \\
\hline 139 & $07: 57: 47.22$ & $-60: 51: 38.92$ & $0.39 \pm 0.06$ & 9.2 & 242.91 & 5.77 & \\
\hline 140 & 07:58:33.36 & $-60: 51: 31$ & $0.7 \pm 0.07$ & 15.44 & 218.55 & 4.73 & \\
\hline 141 & $07: 58: 22.33$ & $-60: 51: 27.9$ & $4.03 \pm 0.15$ & 54.38 & 225.94 & 4.3 & \\
\hline 142 & $07: 57: 28.31$ & $-60: 51: 24.19$ & $2.06 \pm 0.13$ & 25.52 & 213.02 & 7.31 & \\
\hline 143 & 07:56:47.85 & $-60: 51: 9.76$ & $0.61 \pm 0.11$ & 7.48 & 114.54 & 11.61 & \\
\hline 144 & 07:57:39.91 & $-60: 51: 5.08$ & $0.44 \pm 0.08$ & 6.62 & 236.46 & 6 & \\
\hline 145 & $07: 57: 25.43$ & $-60: 51: 1.44$ & $0.24 \pm 0.05$ & 6.83 & 204.78 & 7.4 & \\
\hline 146 & 07:59:9.6 & $-60: 50: 53.48$ & $1.08 \pm 0.09$ & 20.55 & 196.28 & 7.35 & \\
\hline 147 & $07: 57: 49.23$ & $-60: 50: 53.81$ & $0.56 \pm 0.08$ & 9.33 & 222.08 & 5.05 & \\
\hline 148 & 07:59:20.99 & $-60: 50: 53.02$ & $2.05 \pm 0.13$ & 24.98 & 195.36 & 8.58 & \\
\hline 149 & $07: 57: 10.62$ & $-60: 50: 52.58$ & $0.57 \pm 0.09$ & 7.67 & 180.66 & 8.93 & \\
\hline 150 & 07:58:41.79 & $-60: 50: 47.98$ & $1.09 \pm 0.08$ & 17.9 & 265.38 & 4.67 & \\
\hline 151 & $07: 57: 33.56$ & $-60: 50: 42.47$ & $0.34 \pm 0.07$ & 7.16 & 210.8 & 6.39 & \\
\hline 153 & 08:00:4.18 & $-60: 50: 31.45$ & $2.36 \pm 0.22$ & 16.5 & 84.17 & 13.43 & \\
\hline 154 & 07:57:56.73 & $-60: 50: 33.94$ & $1.85 \pm 0.11$ & 28.81 & 191.4 & 4.2 & \\
\hline 155 & 07:59:42.51 & $-60: 50: 28.5$ & $0.4 \pm 0.08$ & 6.34 & 150.04 & 10.88 & \\
\hline 156 & 07:58:15.51 & $-60: 50: 29.44$ & $0.13 \pm 0.03$ & 6.64 & 290.43 & 3.3 & \\
\hline 157 & 07:57:36.04 & $-60: 50: 26.41$ & $0.68 \pm 0.08$ & 12.14 & 220.81 & 5.99 & \\
\hline 158 & 07:58:48.38 & $-60: 50: 25.91$ & $0.57 \pm 0.06$ & 13.39 & 263.85 & 4.97 & \\
\hline 159 & 07:58:36.48 & $-60: 50: 19.25$ & $3.02 \pm 0.12$ & 51.5 & 274.35 & 3.89 & \\
\hline 161 & 07:58:8.08 & $-60: 50: 14.06$ & $0.09 \pm 0.02$ & 6.33 & 287.9 & 3.24 & \\
\hline 162 & $07: 56: 30.54$ & $-60: 50: 10.86$ & $2.03 \pm 0.25$ & 10.96 & 55.51 & 13.37 & \\
\hline 163 & 07:57:29.61 & $-60: 50: 11.58$ & $1.88 \pm 0.11$ & 30.41 & 218.49 & 6.55 & \\
\hline 164 & 07:56:53.39 & $-60: 50: 8.09$ & $0.76 \pm 0.12$ & 8.18 & 138.31 & 10.66 & \\
\hline 165 & 07:58:17.62 & $-60: 50: 5.96$ & $0.35 \pm 0.05$ & 9.65 & 293.55 & 2.9 & \\
\hline 166 & 07:58:11.54 & $-60: 50: 1.43$ & $0.68 \pm 0.07$ & 17.21 & 292.01 & 2.91 & \\
\hline 167 & 07:56:59.36 & $-60: 49: 55.96$ & $1.84 \pm 0.13$ & 22.94 & 157.8 & 9.9 & \\
\hline 168 & 07:58:41.79 & $-60: 49: 57.18$ & $0.33 \pm 0.05$ & 8.33 & 279.63 & 4.05 & \\
\hline 169 & $07: 59: 8.18$ & $-60: 49: 55.49$ & $0.19 \pm 0.04$ & 7.23 & 229.12 & 6.76 & \\
\hline 170 & $07: 57: 43.53$ & $-60: 49: 46.2$ & $0.91 \pm 0.07$ & 19.44 & 255.78 & 4.87 & \\
\hline 171 & $07: 57: 23.26$ & $-60: 49: 39.07$ & $1.8 \pm 0.11$ & 27.66 & 207.43 & 7.04 & \\
\hline 172 & 07:57:57.93 & $-60: 49: 37.02$ & $0.23 \pm 0.04$ & 7.18 & 251.49 & 3.39 & \\
\hline 173 & 07:59:35.43 & $-60: 49: 30.94$ & $0.32 \pm 0.07$ & 5.27 & 173.18 & 9.79 & $\mathrm{U}$ \\
\hline 174 & 07:58:10.64 & $-60: 49: 32.3$ & $0.46 \pm 0.05$ & 13.22 & 295.17 & 2.48 & \\
\hline 175 & 07:59:56.69 & $-60: 49: 29.75$ & $1.04 \pm 0.13$ & 12.49 & 119.94 & 12.32 & \\
\hline 176 & 07:58:50.57 & $-60: 49: 28.67$ & $13.91 \pm 0.25$ & 130.17 & 267.21 & 4.64 & \\
\hline 177 & $07: 59: 2.1$ & $-60: 49: 27.98$ & $0.92 \pm 0.09$ & 14.57 & 219.85 & 5.9 & \\
\hline 178 & 07:58:33.49 & $-60: 49: 25.57$ & $0.23 \pm 0.04$ & 9.28 & 240.52 & 2.96 & \\
\hline 180 & 07:59:47.66 & $-60: 49: 23.16$ & $0.5 \pm 0.08$ & 8.54 & 124.79 & 11.22 & \\
\hline 181 & 07:58:54.42 & $-60: 49: 14.45$ & $0.11 \pm 0.03$ & 5.35 & 264.28 & 4.95 & $\mathrm{U}$ \\
\hline 182 & $07: 57: 34.18$ & $-60: 49: 10.34$ & $0.6 \pm 0.07$ & 12.74 & 238.48 & 5.63 & \\
\hline 183 & 07:57:10.91 & $-60: 49: 9.37$ & $0.79 \pm 0.09$ & 13.41 & 179.36 & 8.34 & \\
\hline 184 & 07:59:35.67 & $-60: 49: 8.62$ & $0.45 \pm 0.07$ & 10.92 & 174.61 & 9.74 & \\
\hline 185 & 07:58:29.74 & $-60: 49: 1.7$ & $3.13 \pm 0.12$ & 54.33 & 296.25 & 2.37 & \\
\hline 186 & $07: 56: 46.22$ & $-60: 48: 58.39$ & $4.29 \pm 0.22$ & 33.1 & 136.46 & 11.26 & \\
\hline 187 & 07:59:17.96 & $-60: 48: 58.93$ & $0.22 \pm 0.05$ & 5.87 & 213.48 & 7.6 & \\
\hline 188 & 07:59:21.29 & $-60: 48: 57.06$ & $5.66 \pm 0.19$ & 59.3 & 201.57 & 7.98 & \\
\hline 189 & $07: 56: 38.58$ & $-60: 48: 52.02$ & $1.58 \pm 0.16$ & 14.34 & 94.92 & 12.17 & \\
\hline 190 & 07:58:32.82 & $-60: 48: 53.03$ & $0.41 \pm 0.05$ & 15.03 & 240.56 & 2.52 & \\
\hline 191 & 07:59:32.03 & $-60: 48: 46.87$ & $8.85 \pm 0.27$ & 75.1 & 162.35 & 9.24 & \\
\hline
\end{tabular}


I. Pillitteri et al.: Deep X-ray survey of NGC 2516 with XMM-Newton, Online Material p 6

Table A.1. continued.

\begin{tabular}{|c|c|c|c|c|c|c|c|}
\hline Num & $\begin{array}{l}\text { RA } \\
\text { J2000 }\end{array}$ & $\begin{array}{l}\text { Dec } \\
\text { J2000 }\end{array}$ & $\begin{array}{l}\text { Count rate } \\
\text { cts ks }^{-1}\end{array}$ & Signif. & $\begin{array}{l}\text { Exp. time } \\
\text { ks }\end{array}$ & $\begin{array}{l}\text { Offaxis } \\
\text { arcmin }\end{array}$ & Un. \\
\hline 192 & $07: 58: 2.67$ & $-60: 48: 47.34$ & $1.18 \pm 0.08$ & 25.64 & 290.03 & 2.4 & \\
\hline 193 & 07:57:19.72 & $-60: 48: 46.55$ & $2.31 \pm 0.13$ & 32.55 & 186.44 & 7.21 & \\
\hline 194 & $07: 57: 46.28$ & $-60: 48: 42.23$ & $0.56 \pm 0.07$ & 10.21 & 264.75 & 4.08 & \\
\hline 195 & 07:58:51.09 & $-60: 48: 38.16$ & $0.23 \pm 0.04$ & 8.21 & 262.96 & 4.35 & $\mathrm{U}$ \\
\hline 196 & $07: 58: 4.95$ & $-60: 48: 33.41$ & $0.25 \pm 0.04$ & 10.91 & 294.87 & 2.04 & \\
\hline 197 & 07:57:30.96 & $-60: 48: 32.62$ & $3.26 \pm 0.15$ & 43.51 & 204.17 & 5.82 & \\
\hline 198 & $07: 58: 23.18$ & $-60: 48: 28.12$ & $0.24 \pm 0.04$ & 7.31 & 302.07 & 1.45 & \\
\hline 199 & $07: 57: 55.48$ & $-60: 48: 27.32$ & $4.34 \pm 0.15$ & 59.22 & 239.54 & 2.95 & \\
\hline 200 & $07: 58: 6.82$ & $-60: 48: 24.73$ & $1.69 \pm 0.1$ & 28.6 & 296.27 & 1.77 & \\
\hline 201 & 07:58:38.9 & $-60: 48: 23.83$ & $0.23 \pm 0.04$ & 9.04 & 214.92 & 2.88 & \\
\hline 202 & $07: 57: 18.65$ & $-60: 48: 15.16$ & $1.66 \pm 0.12$ & 24.46 & 177.07 & 7.25 & \\
\hline 203 & $07: 57: 36.17$ & $-60: 48: 13.5$ & $0.6 \pm 0.07$ & 13.25 & 238.72 & 5.13 & \\
\hline 204 & 07:56:51.64 & $-60: 48: 11.27$ & $0.54 \pm 0.1$ & 6.35 & 150.16 & 10.51 & \\
\hline 205 & 07:58:36.72 & $-60: 48: 6.8$ & $0.84 \pm 0.08$ & 15.91 & 289.11 & 2.52 & \\
\hline 206 & $07: 56: 34.62$ & $-60: 48: 3.89$ & $11.17 \pm 0.45$ & 53.27 & 78.7 & 12.57 & \\
\hline 207 & $07: 57: 9.5$ & $-60: 48: 3.49$ & $0.83 \pm 0.09$ & 13.6 & 184.72 & 8.33 & \\
\hline 208 & 07:59:9.2 & $-60: 47: 57.52$ & $0.44 \pm 0.07$ & 9.23 & 190.87 & 6.36 & \\
\hline 209 & 07:59:27.66 & $-60: 47: 49.2$ & $1.97 \pm 0.13$ & 26.07 & 173.32 & 8.59 & \\
\hline 210 & $07: 59: 2.85$ & $-60: 47: 49.63$ & $0.49 \pm 0.06$ & 11.4 & 244.1 & 5.58 & \\
\hline 211 & 07:58:48.85 & $-60: 47: 48.08$ & $0.62 \pm 0.06$ & 15.41 & 279.05 & 3.88 & \\
\hline 212 & $07: 56: 34.51$ & $-60: 47: 39.66$ & $3.8 \pm 0.29$ & 26.97 & 80.44 & 12.56 & \\
\hline 213 & 07:58:5.31 & $-60: 47: 41.03$ & $0.41 \pm 0.05$ & 10.93 & 297.17 & 1.55 & \\
\hline 214 & 07:59:15.89 & $-60: 47: 35.41$ & $0.6 \pm 0.08$ & 12.67 & 200.48 & 7.14 & \\
\hline 215 & 07:59:49.84 & $-60: 47: 33.9$ & $1.27 \pm 0.12$ & 17.7 & 148.98 & 11.28 & $\mathrm{U}$ \\
\hline 216 & $07: 59: 23.26$ & $-60: 47: 32.86$ & $2.55 \pm 0.13$ & 34.32 & 203.47 & 8.04 & \\
\hline 217 & 07:57:44.11 & $-60: 47: 28.28$ & $2.95 \pm 0.12$ & 47.12 & 254.42 & 4.07 & \\
\hline 218 & 07:59:36.62 & $-60: 47: 24.29$ & $0.18 \pm 0.04$ & 6.19 & 170.23 & 9.67 & \\
\hline 219 & 07:58:31.08 & $-60: 47: 24.9$ & $0.35 \pm 0.05$ & 11.46 & 247.23 & 1.68 & \\
\hline 220 & 07:58:56.49 & $-60: 47: 24$ & $0.83 \pm 0.07$ & 17.92 & 252.5 & 4.77 & \\
\hline 221 & 07:59:1.41 & $-60: 47: 22.31$ & $0.27 \pm 0.05$ & 8.91 & 215.13 & 5.37 & \\
\hline 222 & 07:57:30.89 & $-60: 47: 19.9$ & $0.3 \pm 0.05$ & 8.45 & 222.91 & 5.68 & \\
\hline 223 & 07:56:54.49 & $-60: 47: 18.71$ & $1.06 \pm 0.13$ & 12.79 & 137.9 & 10.12 & \\
\hline 224 & $07: 57: 37.23$ & $-60: 47: 16.94$ & $0.24 \pm 0.05$ & 6.77 & 225.45 & 4.9 & \\
\hline 225 & 07:57:15.94 & $-60: 47: 12.01$ & $0.97 \pm 0.11$ & 12.46 & 180.17 & 7.5 & \\
\hline 226 & 07:59:5.97 & $-60: 47: 7.73$ & $1.2 \pm 0.09$ & 24.14 & 231.67 & 5.92 & $\mathrm{U}$ \\
\hline 227 & 07:56:38.93 & $-60: 47: 5.82$ & $0.41 \pm 0.1$ & 5.55 & 80.03 & 12.02 & \\
\hline 228 & $07: 56: 59.56$ & $-60: 47: 0.53$ & $1.96 \pm 0.14$ & 20.01 & 164.93 & 9.5 & \\
\hline 229 & $07: 57: 52.55$ & $-60: 46: 58.04$ & $0.21 \pm 0.05$ & 5.95 & 258.69 & 3.04 & \\
\hline 230 & 07:58:35.76 & $-60: 46: 53.65$ & $4.02 \pm 0.14$ & 56.6 & 261.89 & 2.26 & \\
\hline 231 & 07:58:16.89 & $-60: 46: 51.64$ & $0.89 \pm 0.07$ & 21.27 & 268.79 & 0.35 & \\
\hline 232 & $07: 58: 8.28$ & $-60: 46: 49.69$ & $0.58 \pm 0.06$ & 14.45 & 293.98 & 1.18 & \\
\hline 233 & 07:58:23.58 & $-60: 46: 49.51$ & $0.32 \pm 0.05$ & 9.28 & 291.63 & 0.84 & \\
\hline 234 & 07:59:21.63 & $-60: 46: 47.32$ & $0.75 \pm 0.08$ & 13.09 & 202.89 & 7.85 & \\
\hline 235 & $07: 57: 25.14$ & $-60: 46: 47.6$ & $1.06 \pm 0.1$ & 14.65 & 192.35 & 6.39 & \\
\hline 236 & $07: 58: 2.45$ & $-60: 46: 47.39$ & $5.04 \pm 0.15$ & 73.96 & 281.77 & 1.87 & \\
\hline 237 & 07:58:5.48 & $-60: 46: 47.32$ & $0.17 \pm 0.04$ & 8.67 & 295.31 & 1.51 & $\mathrm{U}$ \\
\hline 238 & 07:58:31.75 & $-60: 46: 45.98$ & $0.17 \pm 0.03$ & 7.95 & 271.03 & 1.8 & \\
\hline 239 & 07:57:30.97 & $-60: 46: 45.52$ & $0.33 \pm 0.06$ & 7.52 & 209.12 & 5.69 & \\
\hline 240 & 07:57:20.82 & $-60: 46: 44.87$ & $0.33 \pm 0.05$ & 8.63 & 206.71 & 6.92 & \\
\hline 241 & 07:56:38.81 & $-60: 46: 39.9$ & $1.03 \pm 0.14$ & 11.26 & 93.3 & 12.05 & \\
\hline 242 & 07:57:39.73 & $-60: 46: 39.61$ & $1.1 \pm 0.08$ & 22.62 & 246.88 & 4.63 & \\
\hline 243 & 07:58:44.02 & $-60: 46: 37.16$ & $0.22 \pm 0.04$ & 8.04 & 277.44 & 3.3 & \\
\hline 244 & 07:58:20.26 & $-60: 46: 34.61$ & $0.18 \pm 0.04$ & 6.46 & 307.78 & 0.71 & \\
\hline 245 & $07: 56: 47.83$ & $-60: 46: 31.8$ & $4.07 \pm 0.22$ & 31.99 & 135.29 & 10.95 & \\
\hline 246 & 07:58:36.95 & $-60: 46: 27.62$ & $0.46 \pm 0.06$ & 13.85 & 281.05 & 2.5 & \\
\hline 247 & 07:58:33.38 & $-60: 46: 25.93$ & $0.7 \pm 0.06$ & 18.85 & 283.26 & 2.09 & \\
\hline 248 & 08:00:3.87 & $-60: 46: 21.97$ & $3.22 \pm 0.24$ & 20.38 & 88.02 & 13.02 & \\
\hline 249 & 07:58:20.67 & $-60: 46: 21.86$ & $0.41 \pm 0.06$ & 9.09 & 285.1 & 0.93 & \\
\hline 250 & 08:00:16.2 & $-60: 46: 17$ & $1.84 \pm 0.25$ & 9.81 & 61.28 & 14.53 & \\
\hline
\end{tabular}


Table A.1. continued.

\begin{tabular}{|c|c|c|c|c|c|c|c|}
\hline Num & $\begin{array}{l}\text { RA } \\
\text { J2000 }\end{array}$ & $\begin{array}{l}\text { Dec } \\
\text { J2000 }\end{array}$ & $\begin{array}{l}\text { Count rate } \\
\text { cts ks }^{-1}\end{array}$ & Signif. & $\begin{array}{l}\text { Exp. time } \\
\text { ks }\end{array}$ & $\begin{array}{l}\text { Offaxis } \\
\text { arcmin }\end{array}$ & Un. \\
\hline 251 & $07: 59: 6.29$ & $-60: 46: 17.36$ & $0.4 \pm 0.06$ & 9.19 & 214.8 & 6.03 & \\
\hline 252 & 07:58:5.06 & $-60: 46: 12.29$ & $1.79 \pm 0.1$ & 33.08 & 261.96 & 1.81 & \\
\hline 254 & $07: 57: 53.4$ & $-60: 46: 9.91$ & $1.4 \pm 0.1$ & 23.97 & 243.57 & 3.11 & \\
\hline 255 & $07: 57: 29.22$ & $-60: 46: 7.14$ & $0.63 \pm 0.08$ & 13.96 & 208.75 & 5.98 & \\
\hline 256 & 07:59:22.99 & $-60: 46: 6.56$ & $0.16 \pm 0.04$ & 6.79 & 193 & 8.08 & \\
\hline 257 & 07:59:21.69 & $-60: 46: 5.09$ & $1.99 \pm 0.14$ & 23.38 & 185.71 & 7.92 & \\
\hline 258 & 07:56:34.22 & $-60: 45: 59.9$ & $1.27 \pm 0.19$ & 9.7 & 76.86 & 12.65 & \\
\hline 259 & $07: 58: 32.3$ & $-60: 46: 1.52$ & $3.8 \pm 0.14$ & 50.59 & 263.77 & 2.16 & \\
\hline 260 & 07:57:58.33 & $-60: 45: 45.22$ & $0.74 \pm 0.07$ & 18.58 & 255.16 & 2.74 & \\
\hline 261 & 07:57:42.98 & $-60: 45: 40.72$ & $1.51 \pm 0.1$ & 28.93 & 244.51 & 4.47 & \\
\hline 262 & 07:57:26.11 & $-60: 45: 39.42$ & $1.03 \pm 0.1$ & 15.23 & 185.82 & 6.45 & \\
\hline 263 & 07:58:44.07 & $-60: 45: 38.23$ & $0.77 \pm 0.07$ & 17.36 & 250.56 & 3.61 & \\
\hline 264 & 07:59:38.96 & $-60: 45: 36.32$ & $0.24 \pm 0.06$ & 5.28 & 159.08 & 10.08 & \\
\hline 265 & 07:57:49.69 & $-60: 45: 36.18$ & $0.56 \pm 0.06$ & 12.6 & 251.36 & 3.74 & $\mathrm{U}$ \\
\hline 266 & 07:59:53.23 & $-60: 45: 32.04$ & $0.57 \pm 0.12$ & 5.59 & 125.82 & 11.81 & \\
\hline 267 & 07:59:40.91 & $-60: 45: 28.51$ & $1.67 \pm 0.14$ & 17.76 & 156.12 & 10.34 & $\mathrm{U}$ \\
\hline 268 & $07: 57: 2.64$ & $-60: 45: 28.01$ & $1.42 \pm 0.12$ & 19.12 & 166.65 & 9.29 & \\
\hline 269 & 07:59:22.38 & $-60: 45: 27.32$ & $2.1 \pm 0.13$ & 28.73 & 184.81 & 8.12 & \\
\hline 270 & 07:58:13.99 & $-60: 45: 24.66$ & $0.34 \pm 0.04$ & 11.56 & 295.02 & 1.84 & \\
\hline 271 & 07:58:39.02 & $-60: 45: 21.89$ & $0.28 \pm 0.05$ & 8.13 & 286.39 & 3.21 & \\
\hline 272 & 08:00:4.9 & $-60: 45: 19.22$ & $2.18 \pm 0.23$ & 14 & 76.49 & 13.25 & \\
\hline 273 & 07:59:17.69 & $-60: 45: 20.27$ & $1.02 \pm 0.09$ & 17.71 & 211.1 & 7.59 & \\
\hline 274 & 08:00:14.59 & $-60: 45: 17.03$ & $2.73 \pm 0.28$ & 14.4 & 63.91 & 14.43 & \\
\hline 275 & 07:58:18.18 & $-60: 45: 15.98$ & $3.71 \pm 0.13$ & 59.99 & 283.31 & 1.94 & \\
\hline 276 & 07:58:26.98 & $-60: 45: 10.87$ & $0.6 \pm 0.06$ & 13.95 & 260.92 & 2.33 & \\
\hline 277 & 07:57:56.21 & $-60: 45: 9.65$ & $0.08 \pm 0.02$ & 5.07 & 274.52 & 3.3 & \\
\hline 278 & 07:57:43.49 & $-60: 45: 8.5$ & $0.25 \pm 0.05$ & 6.28 & 242.89 & 4.63 & \\
\hline 279 & $07: 57: 54.75$ & $-60: 45: 8.39$ & $1.02 \pm 0.08$ & 16.37 & 264.79 & 3.45 & \\
\hline 280 & $07: 56: 33.6$ & $-60: 45: 3.38$ & $1.46 \pm 0.19$ & 11.51 & 64.62 & 12.85 & \\
\hline 282 & 07:57:12.86 & $-60: 45: 3.38$ & $0.93 \pm 0.11$ & 13.84 & 176.37 & 8.17 & \\
\hline 283 & 07:58:4.96 & $-60: 45: 2.41$ & $0.65 \pm 0.06$ & 14.24 & 282.14 & 2.64 & \\
\hline 284 & $07: 57: 25.4$ & $-60: 45: 0.5$ & $4.37 \pm 0.18$ & 45.79 & 202.66 & 6.72 & \\
\hline 285 & 07:59:47.73 & $-60: 44: 54.38$ & $1.32 \pm 0.14$ & 12.96 & 139.12 & 11.26 & \\
\hline 286 & 07:56:58.47 & $-60: 44: 54.64$ & $0.31 \pm 0.07$ & 6.23 & 152.95 & 9.91 & \\
\hline 287 & 07:59:10.92 & $-60: 44: 47.22$ & $0.93 \pm 0.09$ & 16.74 & 199.44 & 6.96 & \\
\hline 289 & 07:58:33.66 & $-60: 44: 42.18$ & $0.21 \pm 0.04$ & 9.54 & 287.46 & 3.19 & \\
\hline 290 & $07: 58: 8.84$ & $-60: 44: 40.06$ & $3.35 \pm 0.13$ & 46.74 & 265.18 & 2.74 & \\
\hline 291 & 07:58:17.41 & $-60: 44: 38.18$ & $0.29 \pm 0.05$ & 5.79 & 252.64 & 2.57 & \\
\hline 292 & 07:59:36.59 & $-60: 44: 33.5$ & $8.91 \pm 0.28$ & 61.96 & 159.45 & 10.02 & \\
\hline 293 & 07:59:59.39 & $-60: 44: 29.26$ & $0.29 \pm 0.07$ & 6.7 & 100.61 & 12.74 & \\
\hline 294 & 07:58:27.97 & $-60: 44: 31.24$ & $0.31 \pm 0.05$ & 8.87 & 253.93 & 2.97 & \\
\hline 295 & 07:58:33.53 & $-60: 44: 30.52$ & $4.94 \pm 0.15$ & 62.45 & 285.06 & 3.33 & \\
\hline 296 & 07:59:5.14 & $-60: 44: 27.56$ & $0.81 \pm 0.08$ & 16.71 & 202.68 & 6.44 & \\
\hline 297 & 07:58:21.14 & $-60: 44: 25.3$ & $0.23 \pm 0.04$ & 8.24 & 282.62 & 2.82 & \\
\hline 298 & $07: 57: 42.83$ & $-60: 44: 20.65$ & $1.25 \pm 0.09$ & 23.98 & 243.53 & 5.1 & \\
\hline 299 & $07: 57: 39.33$ & $-60: 44: 15.76$ & $0.19 \pm 0.04$ & 8.04 & 209.34 & 5.5 & $\mathrm{U}$ \\
\hline 300 & 07:57:57.41 & $-60: 44: 15.68$ & $2.24 \pm 0.11$ & 39.23 & 267.02 & 3.82 & \\
\hline 301 & 07:58:50.43 & $-60: 44: 14.57$ & $0.21 \pm 0.05$ & 5.4 & 257.22 & 5 & $\mathrm{U}$ \\
\hline 302 & 07:58:26.01 & $-60: 44: 13.24$ & $0.2 \pm 0.04$ & 8.67 & 287.2 & 3.16 & \\
\hline 303 & 07:57:9.98 & $-60: 44: 11.58$ & $1.23 \pm 0.12$ & 15.09 & 171.23 & 8.77 & \\
\hline 304 & 07:59:0.72 & $-60: 44: 11.76$ & $0.37 \pm 0.06$ & 8.41 & 222.52 & 6.08 & \\
\hline 305 & 07:57:20.82 & $-60: 44: 3.8$ & $1.95 \pm 0.12$ & 28.13 & 190.75 & 7.59 & \\
\hline 306 & 07:58:22.61 & $-60: 44: 4.13$ & $0.59 \pm 0.07$ & 13.78 & 278.19 & 3.2 & \\
\hline 307 & 07:57:1.08 & $-60: 43: 46.85$ & $1.02 \pm 0.11$ & 14.09 & 154.8 & 9.93 & $\mathrm{U}$ \\
\hline 308 & 07:59:2.9 & $-60: 43: 46.27$ & $0.38 \pm 0.05$ & 11.1 & 230.18 & 6.53 & \\
\hline 309 & 07:57:5.71 & $-60: 43: 40.94$ & $0.44 \pm 0.08$ & 6.74 & 144.62 & 9.44 & \\
\hline 310 & 07:59:50.05 & $-60: 43: 35.69$ & $0.2 \pm 0.07$ & 5.01 & 134.29 & 11.87 & \\
\hline 311 & 07:58:14.63 & $-60: 43: 33.1$ & $0.94 \pm 0.08$ & 19.78 & 241.54 & 3.67 & \\
\hline 312 & $07: 59: 2.15$ & $-60: 43: 25.64$ & $0.28 \pm 0.05$ & 8.11 & 223.79 & 6.64 & \\
\hline
\end{tabular}


I. Pillitteri et al.: Deep X-ray survey of NGC 2516 with XMM-Newton, Online Material p 8

Table A.1. continued.

\begin{tabular}{|c|c|c|c|c|c|c|c|}
\hline Num & $\begin{array}{l}\text { RA } \\
\text { J2000 }\end{array}$ & $\begin{array}{l}\text { Dec } \\
\text { J2000 }\end{array}$ & $\begin{array}{l}\text { Count rate } \\
\mathrm{cts} \mathrm{ks}^{-1}\end{array}$ & Signif. & $\begin{array}{l}\text { Exp. time } \\
\text { ks }\end{array}$ & $\begin{array}{l}\text { Offaxis } \\
\text { arcmin }\end{array}$ & Un. \\
\hline 313 & $07: 56: 47.14$ & $-60: 43: 22.4$ & $1.86 \pm 0.17$ & 18.96 & 95.02 & 11.67 & \\
\hline 314 & 07:59:51.66 & $-60: 43: 21.65$ & $13.23 \pm 0.39$ & 71.49 & 126.17 & 12.13 & \\
\hline 315 & 07:57:36.11 & $-60: 43: 21.72$ & $0.29 \pm 0.06$ & 5.97 & 200.01 & 6.34 & \\
\hline 316 & $07: 57: 52.61$ & $-60: 43: 19.49$ & $1.46 \pm 0.11$ & 20.74 & 221.21 & 4.92 & \\
\hline 317 & 07:58:39.77 & $-60: 43: 16.68$ & $0.85 \pm 0.08$ & 15.73 & 262.49 & 4.78 & \\
\hline 318 & 07:59:34.35 & $-60: 43: 11.78$ & $0.37 \pm 0.07$ & 6.28 & 162.52 & 10.21 & $\mathrm{U}$ \\
\hline 319 & 07:58:22.15 & $-60: 43: 12.07$ & $0.27 \pm 0.05$ & 7.53 & 272.96 & 4.04 & \\
\hline 320 & 07:59:39.19 & $-60: 43: 8.04$ & $0.25 \pm 0.06$ & 5.08 & 155.1 & 10.78 & \\
\hline 321 & 07:58:41.69 & $-60: 43: 9.37$ & $0.17 \pm 0.04$ & 6.01 & 258.73 & 5.01 & \\
\hline 322 & 07:57:45.54 & $-60: 43: 8.33$ & $0.51 \pm 0.07$ & 10.62 & 214.93 & 5.63 & \\
\hline 323 & 07:58:57.58 & $-60: 42: 59.69$ & $7.58 \pm 0.21$ & 80.27 & 225.83 & 6.46 & \\
\hline 324 & $07: 58: 1.65$ & $-60: 42: 53.03$ & $0.94 \pm 0.08$ & 16.34 & 251.95 & 4.73 & \\
\hline 325 & 07:58:53.63 & $-60: 42: 51.3$ & $0.19 \pm 0.04$ & 5.86 & 237.45 & 6.2 & \\
\hline 326 & 07:57:53.85 & $-60: 42: 48.82$ & $0.66 \pm 0.07$ & 17.37 & 243.92 & 5.25 & $\mathrm{U}$ \\
\hline 327 & 08:00:20.75 & $-60: 42: 36.4$ & $3.02 \pm 0.5$ & 10.27 & 19.42 & 15.75 & \\
\hline 328 & 07:59:29.87 & $-60: 42: 37.01$ & $0.61 \pm 0.09$ & 10.39 & 168.59 & 9.97 & \\
\hline 329 & 07:57:47.25 & $-60: 42: 37.98$ & $1.87 \pm 0.11$ & 25.24 & 231.32 & 5.87 & \\
\hline 330 & 07:58:39.93 & $-60: 42: 35.14$ & $0.89 \pm 0.08$ & 18.36 & 253.09 & 5.37 & $\mathrm{U}$ \\
\hline 331 & 07:59:40.11 & $-60: 42: 31.25$ & $2.63 \pm 0.18$ & 28.12 & 127.62 & 11.13 & \\
\hline 332 & 07:57:56.18 & $-60: 42: 31.93$ & $1.7 \pm 0.11$ & 22.98 & 242.74 & 5.34 & \\
\hline 333 & 07:57:12.79 & $-60: 42: 23.76$ & $0.56 \pm 0.1$ & 6.61 & 146.37 & 9.24 & \\
\hline 334 & 07:59:11.83 & $-60: 42: 22.46$ & $4.04 \pm 0.17$ & 48.75 & 189.77 & 8.21 & \\
\hline 336 & 07:58:49.57 & $-60: 42: 13.9$ & $2.1 \pm 0.12$ & 30.92 & 209.03 & 6.34 & \\
\hline 337 & 07:57:51.3 & $-60: 42: 12.67$ & $0.49 \pm 0.07$ & 10.31 & 229.8 & 5.92 & \\
\hline 338 & $07: 57: 26.74$ & $-60: 42: 11.48$ & $0.62 \pm 0.09$ & 8.78 & 188.27 & 7.96 & \\
\hline 339 & 07:57:31.38 & $-60: 42: 10.66$ & $0.25 \pm 0.05$ & 7.01 & 198.54 & 7.54 & \\
\hline 340 & 07:58:53.98 & $-60: 42: 8.14$ & $0.67 \pm 0.07$ & 13.58 & 217.1 & 6.75 & \\
\hline 341 & 07:58:34.83 & $-60: 42: 6.12$ & $0.34 \pm 0.05$ & 9.98 & 247.87 & 5.53 & \\
\hline 342 & 07:59:55.79 & $-60: 41: 59.39$ & $2.97 \pm 0.22$ & 22.13 & 112.97 & 13.1 & \\
\hline 343 & 07:58:19.97 & $-60: 41: 58.16$ & $3.42 \pm 0.14$ & 49.59 & 225.66 & 5.24 & \\
\hline 344 & 07:57:49.01 & $-60: 41: 55.64$ & $0.62 \pm 0.07$ & 13.79 & 195.48 & 6.31 & \\
\hline 346 & 07:58:30.25 & $-60: 41: 50.5$ & $3.79 \pm 0.15$ & 51.78 & 228.89 & 5.58 & \\
\hline 347 & 07:58:40.45 & $-60: 41: 45.96$ & $1.97 \pm 0.12$ & 26.95 & 239.31 & 6.12 & \\
\hline 348 & 07:58:43.28 & $-60: 41: 45.6$ & $0.65 \pm 0.08$ & 12.93 & 235.91 & 6.29 & \\
\hline 349 & 07:58:45.64 & $-60: 41: 41.53$ & $0.21 \pm 0.04$ & 8.66 & 233.29 & 6.5 & \\
\hline 350 & 07:58:20.36 & $-60: 41: 39.05$ & $0.31 \pm 0.06$ & 9.37 & 233.35 & 5.56 & \\
\hline 351 & 07:59:5.8 & $-60: 41: 26.52$ & $0.26 \pm 0.06$ & 5.68 & 198.54 & 8.25 & \\
\hline 352 & $07: 56: 24.15$ & $-60: 41: 21.23$ & $3.73 \pm 0.71$ & 10.89 & 12.46 & 15.03 & \\
\hline 353 & 07:59:15.66 & $-60: 41: 20.76$ & $0.83 \pm 0.11$ & 10.77 & 174.03 & 9.22 & \\
\hline 354 & 07:59:23.84 & $-60: 41: 18.92$ & $0.9 \pm 0.11$ & 11 & 164.45 & 10.03 & \\
\hline 355 & 07:59:53.05 & $-60: 41: 17.63$ & $0.8 \pm 0.14$ & 7.17 & 115.23 & 13.09 & \\
\hline 356 & 07:58:3.78 & $-60: 41: 13.52$ & $0.46 \pm 0.07$ & 9.12 & 202.41 & 6.2 & $\mathrm{U}$ \\
\hline 357 & 07:57:32.66 & $-60: 41: 11.8$ & $2.16 \pm 0.14$ & 22.4 & 169.59 & 8.12 & $\mathrm{U}$ \\
\hline 358 & 07:57:47.65 & $-60: 41: 11.62$ & $0.73 \pm 0.09$ & 10.47 & 197.55 & 7.02 & \\
\hline 360 & 07:58:57.73 & $-60: 41: 0.13$ & $0.56 \pm 0.07$ & 11.26 & 204.22 & 7.92 & \\
\hline 361 & 08:00:1.87 & $-60: 40: 57.58$ & $0.2 \pm 0.06$ & 5.22 & 82.92 & 14.21 & \\
\hline 362 & $07: 57: 9.28$ & $-60: 40: 54.26$ & $0.49 \pm 0.09$ & 7.44 & 135.48 & 10.44 & \\
\hline 363 & 07:57:14.22 & $-60: 40: 53$ & $4.18 \pm 0.2$ & 43.14 & 147.67 & 9.98 & \\
\hline 364 & 07:57:18.48 & $-60: 40: 52.68$ & $0.5 \pm 0.07$ & 10.01 & 155.73 & 9.58 & \\
\hline 365 & $07: 59: 40.6$ & $-60: 40: 47.5$ & $0.32 \pm 0.09$ & 5.28 & 112.57 & 12.02 & $\mathrm{U}$ \\
\hline 366 & $07: 58: 0.54$ & $-60: 40: 44.11$ & $1.38 \pm 0.11$ & 25.18 & 179.85 & 6.79 & $\mathrm{U}$ \\
\hline 367 & 07:57:55.72 & $-60: 40: 39.79$ & $1.31 \pm 0.1$ & 22.68 & 214.6 & 7.06 & \\
\hline 368 & 08:00:5.07 & $-60: 40: 31.44$ & $1.17 \pm 0.22$ & 6.88 & 53.08 & 14.75 & \\
\hline 369 & 07:59:31.05 & $-60: 40: 27.16$ & $0.17 \pm 0.05$ & 5.62 & 119.16 & 11.25 & \\
\hline 370 & 07:59:13.56 & $-60: 40: 25.07$ & $0.47 \pm 0.07$ & 10.21 & 157.02 & 9.65 & \\
\hline 371 & $07: 58: 42.66$ & $-60: 40: 22.12$ & $0.53 \pm 0.09$ & 7.16 & 178.93 & 7.5 & \\
\hline 372 & 07:58:23.02 & $-60: 40: 21$ & $5.8 \pm 0.19$ & 63.43 & 215.54 & 6.89 & \\
\hline 373 & $07: 58: 2.58$ & $-60: 40: 14.48$ & $0.94 \pm 0.09$ & 15.37 & 210.84 & 7.19 & \\
\hline 374 & 07:58:33.48 & $-60: 40: 13.48$ & $0.35 \pm 0.06$ & 7.29 & 192.3 & 7.25 & $\mathrm{U}$ \\
\hline
\end{tabular}


Table A.1. continued.

\begin{tabular}{|c|c|c|c|c|c|c|c|}
\hline Num & $\begin{array}{l}\text { RA } \\
\text { J2000 }\end{array}$ & $\begin{array}{l}\text { Dec } \\
\text { J2000 }\end{array}$ & $\begin{array}{l}\text { Count rate } \\
\text { cts ks }^{-1}\end{array}$ & Signif. & $\begin{array}{l}\text { Exp. time } \\
\text { ks }\end{array}$ & $\begin{array}{l}\text { Offaxis } \\
\text { arcmin }\end{array}$ & Un. \\
\hline 375 & 08:00:0 & $-60: 40: 9.66$ & $0.92 \pm 0.19$ & 6.95 & 49.79 & 14.38 & \\
\hline 376 & 07:58:8.15 & $-60: 40: 9.8$ & $0.2 \pm 0.05$ & 6.16 & 198.9 & 7.13 & \\
\hline 377 & 07:58:17.16 & $-60: 40: 6.56$ & $0.3 \pm 0.07$ & 6.32 & 185.92 & 7.09 & \\
\hline 378 & $07: 56: 59.13$ & $-60: 39: 58.61$ & $0.39 \pm 0.1$ & 5 & 94.95 & 11.99 & \\
\hline 379 & 07:57:47.75 & $-60: 39: 59.83$ & $0.34 \pm 0.07$ & 7.11 & 124.98 & 8.07 & \\
\hline 380 & 07:56:55.67 & $-60: 39: 52.67$ & $0.16 \pm 0.05$ & 5.04 & 92.59 & 12.39 & \\
\hline 381 & $07: 57: 24.36$ & $-60: 39: 51.48$ & $1.15 \pm 0.12$ & 16.21 & 154.19 & 9.8 & \\
\hline 383 & $07: 57: 25.96$ & $-60: 39: 48.35$ & $2.46 \pm 0.16$ & 24.83 & 157.79 & 9.71 & \\
\hline 384 & 07:59:28.97 & $-60: 39: 40.57$ & $1.28 \pm 0.14$ & 12.99 & 121.71 & 11.54 & \\
\hline 385 & 07:57:10.76 & $-60: 39: 36.14$ & $1.03 \pm 0.13$ & 13.33 & 107.16 & 11.14 & \\
\hline 386 & 07:58:20.69 & $-60: 39: 36.25$ & $0.61 \pm 0.08$ & 10.9 & 175.56 & 7.61 & $\mathrm{U}$ \\
\hline 387 & 07:56:55.51 & $-60: 39: 34.2$ & $0.71 \pm 0.14$ & 6.88 & 90.61 & 12.59 & \\
\hline 388 & 07:58:42.72 & $-60: 39: 33.88$ & $0.5 \pm 0.07$ & 10.52 & 192.15 & 8.24 & \\
\hline 389 & 07:58:52.46 & $-60: 39: 33.26$ & $0.49 \pm 0.08$ & 8.15 & 154.02 & 8.77 & \\
\hline 390 & 07:58:37.74 & $-60: 39: 22.25$ & $0.42 \pm 0.08$ & 6.88 & 164.73 & 8.22 & \\
\hline 391 & $07: 57: 28.74$ & $-60: 39: 10.94$ & $0.37 \pm 0.08$ & 6.04 & 139.96 & 9.99 & \\
\hline 392 & 07:57:46.16 & $-60: 39: 8.5$ & $0.26 \pm 0.06$ & 6.04 & 157.08 & 8.92 & $\mathrm{U}$ \\
\hline 393 & 07:56:38.94 & $-60: 39: 4.07$ & $1.18 \pm 0.23$ & 7.65 & 39.83 & 14.53 & \\
\hline 394 & 07:59:7.75 & $-60: 39: 4.25$ & $0.89 \pm 0.1$ & 14.01 & 153.66 & 10.2 & \\
\hline 395 & 07:58:46.21 & $-60: 39: 2.52$ & $0.22 \pm 0.05$ & 5.09 & 188.34 & 8.89 & \\
\hline 396 & 07:57:15.77 & $-60: 38: 53.34$ & $1.99 \pm 0.18$ & 17.3 & 107.24 & 11.22 & \\
\hline 397 & 07:58:36.21 & $-60: 38: 46.1$ & $1.1 \pm 0.1$ & 16.44 & 180.67 & 8.74 & \\
\hline 398 & $07: 58: 12.53$ & $-60: 38: 38.18$ & $1.69 \pm 0.13$ & 18.66 & 185.12 & 8.59 & \\
\hline 399 & 07:58:50.49 & $-60: 38: 37.9$ & $17.99 \pm 0.37$ & 105.34 & 169.12 & 9.48 & \\
\hline 400 & 07:57:40.72 & $-60: 38: 31.27$ & $0.51 \pm 0.1$ & 6.72 & 104.33 & 9.77 & \\
\hline 401 & 07:59:7.72 & $-60: 38: 30.12$ & $0.29 \pm 0.06$ & 5.82 & 132.1 & 10.65 & \\
\hline 402 & 07:57:30.98 & $-60: 38: 26.45$ & $1.38 \pm 0.15$ & 14.48 & 110.62 & 10.44 & \\
\hline 403 & 07:58:4.35 & $-60: 38: 26.88$ & $0.83 \pm 0.1$ & 11.28 & 132.88 & 8.9 & \\
\hline 404 & 07:59:22.15 & $-60: 38: 23.32$ & $0.44 \pm 0.09$ & 6.79 & 128.88 & 11.85 & \\
\hline 405 & 07:58:34.06 & $-60: 38: 17.27$ & $0.55 \pm 0.08$ & 9.16 & 165.47 & 9.14 & \\
\hline 406 & 07:57:20.04 & $-60: 38: 11.11$ & $0.63 \pm 0.12$ & 7.19 & 104.47 & 11.43 & \\
\hline 407 & $07: 57: 52.14$ & $-60: 38: 8.38$ & $0.41 \pm 0.09$ & 6.11 & 133.56 & 9.57 & \\
\hline 408 & 07:58:13.05 & $-60: 38: 6.61$ & $0.21 \pm 0.05$ & 6.12 & 145.54 & 9.11 & \\
\hline 409 & 07:58:31.01 & $-60: 37: 46.74$ & $1.76 \pm 0.14$ & 24.95 & 135.62 & 9.57 & \\
\hline 410 & 07:59:49.56 & $-60: 37: 44.54$ & $2.53 \pm 0.25$ & 15.35 & 67.61 & 14.71 & $\mathrm{U}$ \\
\hline 411 & 07:56:58.15 & $-60: 37: 45.01$ & $0.74 \pm 0.15$ & 7.25 & 60.81 & 13.54 & \\
\hline 412 & 07:59:55.85 & $-60: 37: 43.82$ & $0.53 \pm 0.14$ & 5.36 & 44.16 & 15.32 & \\
\hline 413 & $07: 57: 32.27$ & $-60: 37: 21.36$ & $0.87 \pm 0.11$ & 14.03 & 105.73 & 11.29 & \\
\hline 414 & $07: 58: 23.55$ & $-60: 37: 20.06$ & $2.99 \pm 0.17$ & 34.31 & 128.72 & 9.9 & \\
\hline 415 & 07:58:19.39 & $-60: 37: 18.16$ & $0.31 \pm 0.08$ & 5.68 & 118.93 & 9.9 & \\
\hline 416 & 07:58:48.65 & $-60: 37: 15.02$ & $0.53 \pm 0.1$ & 6.41 & 127.15 & 10.66 & \\
\hline 417 & 07:58:32.1 & $-60: 37: 14.77$ & $0.4 \pm 0.08$ & 6.76 & 133.63 & 10.12 & \\
\hline 418 & 07:59:22.41 & $-60: 37: 10.81$ & $0.59 \pm 0.11$ & 7.34 & 91.59 & 12.79 & \\
\hline 419 & 07:59:4.76 & $-60: 37: 8.54$ & $0.58 \pm 0.1$ & 8.26 & 115.3 & 11.61 & \\
\hline 420 & $07: 56: 36.65$ & $-60: 36: 47.84$ & $6.79 \pm 0.8$ & 17.26 & 21.09 & 16.13 & \\
\hline 422 & $07: 57: 50.23$ & $-60: 36: 34.81$ & $0.17 \pm 0.05$ & 5.96 & 113.36 & 11.13 & \\
\hline 423 & $07: 57: 47.84$ & $-60: 36: 34.52$ & $2.48 \pm 0.19$ & 21.57 & 112.29 & 11.23 & \\
\hline 425 & 07:58:57.63 & $-60: 36: 13.36$ & $2.56 \pm 0.19$ & 23.82 & 111.21 & 12.03 & \\
\hline 426 & 07:58:53.28 & $-60: 36: 11.2$ & $3.47 \pm 0.22$ & 30.42 & 110.78 & 11.86 & \\
\hline 427 & 07:57:29.36 & $-60: 36: 3.02$ & $1.18 \pm 0.15$ & 12.82 & 80.36 & 12.61 & \\
\hline 428 & 07:57:55.14 & $-60: 36: 0.11$ & $2.02 \pm 0.17$ & 17.81 & 108.16 & 11.53 & \\
\hline 429 & 07:58:14.54 & $-60: 35: 58.42$ & $2 \pm 0.16$ & 22.19 & 117.31 & 11.23 & $\mathrm{U}$ \\
\hline 430 & $07: 58: 6.3$ & $-60: 35: 53.16$ & $13.57 \pm 0.4$ & 80.24 & 113.98 & 11.4 & \\
\hline 431 & 07:58:24.01 & $-60: 35: 48.37$ & $1.2 \pm 0.16$ & 10.91 & 91.3 & 11.42 & \\
\hline 432 & 07:58:18.2 & $-60: 35: 38.08$ & $0.41 \pm 0.09$ & 6.16 & 110.51 & 11.57 & \\
\hline 433 & $07: 58: 11.5$ & $-60: 35: 31.99$ & $0.37 \pm 0.08$ & 6.97 & 111.82 & 11.69 & \\
\hline 434 & 07:58:51.97 & $-60: 35: 20.69$ & $5.97 \pm 0.29$ & 37.16 & 105.24 & 12.59 & \\
\hline 435 & 07:58:41.92 & $-60: 35: 16.8$ & $0.61 \pm 0.12$ & 6.31 & 99.38 & 12.29 & $\mathrm{U}$ \\
\hline 436 & 07:58:16.44 & $-60: 35: 6.54$ & $0.3 \pm 0.07$ & 5.59 & 107.99 & 12.09 & \\
\hline
\end{tabular}


I. Pillitteri et al.: Deep X-ray survey of NGC 2516 with XMM-Newton, Online Material p 10

Table A.1. continued.

\begin{tabular}{llllllll}
\hline \hline Num & RA & Dec & $\begin{array}{l}\text { Count rate } \\
\text { cts ks }^{-1}\end{array}$ & Signif. & $\begin{array}{l}\text { Exp. time } \\
\text { ks }\end{array}$ & $\begin{array}{l}\text { Offaxis } \\
\text { arcmin }\end{array}$ & Un. \\
\hline 437 & $07: 58: 4.74$ & $-60: 35: 4.81$ & $0.84 \pm 0.12$ & 10.19 & 105.08 & 12.22 & \\
439 & $07: 59: 19.8$ & $-60: 34: 43.82$ & $12.96 \pm 0.51$ & 50.19 & 73.5 & 14.62 & \\
440 & $07: 57: 24.66$ & $-60: 34: 33.89$ & $0.46 \pm 0.12$ & 6.28 & 65.16 & 14.19 & \\
441 & $07: 57: 47.03$ & $-60: 34: 33.92$ & $1.01 \pm 0.14$ & 10.62 & 76.28 & 13.17 & \\
442 & $07: 59: 24.3$ & $-60: 34: 32.34$ & $0.5 \pm 0.11$ & 7.36 & 61.2 & 15.08 & \\
444 & $07: 58: 14.87$ & $-60: 34: 19.42$ & $0.5 \pm 0.11$ & 6.22 & 97.79 & 12.88 & U \\
445 & $07: 57: 23.92$ & $-60: 34: 17.83$ & $1.55 \pm 0.24$ & 9.41 & 54.3 & 14.47 & \\
446 & $07: 58: 32.21$ & $-60: 34: 11.96$ & $0.48 \pm 0.11$ & 7.13 & 96.96 & 13.13 & \\
447 & $07: 57: 31.02$ & $-60: 34: 8.76$ & $1.28 \pm 0.21$ & 7.74 & 45.3 & 14.24 & \\
448 & $07: 58: 10.21$ & $-60: 34: 0.34$ & $0.54 \pm 0.09$ & 8.39 & 95.92 & 13.23 & \\
449 & $07: 58: 55.73$ & $-60: 33: 45.83$ & $1.27 \pm 0.19$ & 11.93 & 61.5 & 14.23 & \\
450 & $07: 58: 2.77$ & $-60: 33: 43.49$ & $2.08 \pm 0.2$ & 16.56 & 90.51 & 13.6 & $\mathrm{U}$ \\
451 & $07: 58: 50.88$ & $-60: 33: 31.03$ & $0.89 \pm 0.14$ & 8.99 & 72.22 & 14.28 & \\
453 & $07: 57: 12.74$ & $-60: 33: 6.7$ & $4.32 \pm 0.62$ & 16.55 & 21.34 & 16.16 & \\
455 & $07: 59: 8.82$ & $-60: 33: 1.22$ & $0.72 \pm 0.16$ & 6.73 & 58.63 & 15.52 & \\
456 & $07: 58: 43.69$ & $-60: 32: 56.4$ & $4.13 \pm 0.3$ & 26.79 & 67.19 & 14.62 & \\
459 & $07: 58: 36.65$ & $-60: 32: 20.76$ & $0.79 \pm 0.17$ & 5.66 & 66.53 & 15.04 & $\mathrm{U}$ \\
460 & $07: 58: 42.41$ & $-60: 32: 18.96$ & $0.96 \pm 0.16$ & 8.46 & 65.62 & 15.2 & \\
461 & $07: 59: 11.91$ & $-60: 31: 38.96$ & $2.26 \pm 0.53$ & 7.71 & 16.16 & 16.92 & \\
462 & $07: 57: 47.61$ & $-60: 31: 33.89$ & $3.93 \pm 0.66$ & 10.79 & 16.38 & 16.06 & \\
464 & $07: 57: 36.22$ & $-60: 30: 16.38$ & $3.34 \pm 0.65$ & 8.64 & 14.93 & 17.67 & $\mathrm{U}$ \\
\hline
\end{tabular}


I. Pillitteri et al.: Deep X-ray survey of NGC 2516 with XMM-Newton, Online Material p 11

Appendix B: Identifications, X-ray fluxes and luminosities of NGC 2516 stars 
I. Pillitteri et al.: Deep X-ray survey of NGC 2516 with XMM-Newton, Online Material p 12

Table B.1. Identifications, X-ray fluxes and luminosities of NGC 2516 X-ray detected stars. Columns report: X source identifier (as in Table A.1, identifier from Jeffries et al. 2001), match distance, $V, B-V, V-I$, fluxes, luminosities in the 0.3-7.9 $\mathrm{keV}$ band, $\log L_{\mathrm{bol}}$ and the ratio $\log L_{\mathrm{X}} / L_{\mathrm{bol}}$. Errors are propagated from count rates, for systematic errors see discussion in Sect. 2.2. Exposure times are calculated from the summed exposures, scaling pn to MOS with a factor of 3.6 as described in the text.

\begin{tabular}{|c|c|c|c|c|c|c|c|c|c|}
\hline X-ray Src & Opt. Id. & $\begin{array}{l}\text { Distance } \\
\text { arcsec }\end{array}$ & $\begin{array}{l}V \\
\text { mag }\end{array}$ & $\begin{array}{l}B-V \\
\text { mag }\end{array}$ & $\begin{array}{l}V-I \\
\text { mag }\end{array}$ & $\begin{array}{l}f_{\mathrm{X}}(0.3-7.9 \mathrm{keV}) \\
10^{-15} \mathrm{erg} \mathrm{s}^{-1} \mathrm{~cm}^{-2}\end{array}$ & $\begin{array}{l}\log L_{\mathrm{X}} \\
\mathrm{erg} \mathrm{s}^{-1}\end{array}$ & $\begin{array}{l}\log L_{\text {bol }} \\
\mathrm{erg} \mathrm{s}^{-1}\end{array}$ & $\log L_{\mathrm{X}} / L_{\mathrm{bol}}$ \\
\hline 6 & 6160 & 1 & 20.9 & $\ldots$ & 3.06 & $67 \pm 7$ & $30.08_{-0.05}^{0.04}$ & 31.52 & $\begin{array}{l}-1.44 \\
\end{array}$ \\
\hline 7 & 7036 & 3.8 & 13 & 0.7 & 0.81 & $6 \pm 2$ & $29.02_{-0.1}^{0.103}$ & 33.9 & -4.88 \\
\hline 8 & 9114 & 5.9 & 13.1 & 0.7 & 0.8 & $18 \pm 3$ & $29.52_{-0.07}^{0.06}$ & 33.93 & -4.41 \\
\hline 10 & 8793 & 2.3 & 20.1 & $\ldots$ & 3.02 & $11 \pm 2$ & $29.29_{-0.08}^{0.07}$ & 31.54 & -2.24 \\
\hline 11 & 9544 & 3.5 & 17.5 & 1.5 & 2.25 & $6.5 \pm 2$ & $29.07_{-0.2}^{0.1}$ & 31.94 & -2.87 \\
\hline 12 & 6976 & 4.3 & 12.8 & 0.6 & 0.78 & $46 \pm 4$ & $29.91_{-0.04}^{0.03}$ & 33.97 & -4.05 \\
\hline 15 & 6871 & 5.4 & 14.8 & 1.2 & 1.34 & $7 \pm 2$ & $29.1_{-0.1}^{0.1}$ & 32.93 & -3.83 \\
\hline 16 & 7682 & 1.3 & 19.4 & $\ldots$ & 2.64 & $10 \pm 2$ & $29.25_{-0.09}^{0.07}$ & 31.68 & -2.43 \\
\hline 29 & 9015 & 3.7 & 16.8 & 1.5 & 1.76 & $10.1 \pm 1$ & $29.26_{-0.07}^{0.06}$ & 32.55 & -3.29 \\
\hline 31 & 8126 & 2.6 & 15.6 & 1.2 & 1.36 & $3.7 \pm 0.8$ & $28.82_{-0.1}^{0.09}$ & 32.91 & -4.09 \\
\hline 32 & 6689 & 2.1 & 10.9 & 0.3 & -10 & $52 \pm 4$ & $29.97_{-0.03}^{0.03}$ & 34.5 & -4.53 \\
\hline 38 & 8584 & 2 & 14.7 & 1.2 & 1.29 & $13.9 \pm 1$ & $29.4_{-0.05}^{0.04}$ & 32.97 & -3.58 \\
\hline 39 & 11240 & 2.3 & 15 & 1 & 1.18 & $20.5 \pm 3$ & $29.56_{-0.06}^{0.05}$ & 33.12 & -3.55 \\
\hline 41 & 8037 & 3.1 & 19.7 & $\ldots$ & 2.81 & $3.6 \pm 0.8$ & $28.81_{-0.1}^{0.08}$ & 31.6 & -2.79 \\
\hline 43 & 8990 & 2.8 & 16.2 & 1.3 & 1.55 & $3.9 \pm 0.8$ & $28.85_{-0.1}^{0.08}$ & 32.74 & -3.89 \\
\hline 46 & 5299 & 2.3 & 17.3 & 1.5 & 2.01 & $11.0 \pm 2$ & $29.28_{-0.08}^{0.07}$ & 32.19 & -2.91 \\
\hline 59 & 10440 & 2.5 & 17.2 & 1.5 & 1.92 & $6.1 \pm 1$ & $29.03_{-0.08}^{0.07}$ & 32.32 & -3.29 \\
\hline 60 & 7743 & 1 & 12.6 & 0.7 & 0.8 & $30.9 \pm 1$ & $29.74_{-0.02}^{0.02}$ & 33.93 & -4.18 \\
\hline 61 & 6465 & 2.4 & 15.1 & 1.3 & 1.41 & $18 \pm 2$ & $29.5_{-0.05}^{0.04}$ & 32.87 & -3.37 \\
\hline 63 & 5052 & 2.6 & 10.9 & 0.3 & 0.45 & $67 \pm 5$ & $30.08_{-0.04}^{0.03}$ & 34.48 & -4.4 \\
\hline 65 & 9911 & 1.5 & 18.4 & 1.6 & 2.62 & $9.2 \pm 1$ & $29.22_{-0.05}^{0.04}$ & 31.68 & -2.47 \\
\hline 68 & 7194 & 1.6 & 11.4 & 0.4 & 0.57 & $2.9 \pm 0.7$ & $28.71_{-0.1}^{0.09}$ & 34.32 & -5.61 \\
\hline 69 & 7312 & 6.7 & 13.8 & 0.8 & 0.92 & $6.8 \pm 1$ & $29.09_{-0.07}^{0.06}$ & 33.63 & -4.54 \\
\hline 74 & 6514 & 0.4 & 16.4 & 1.4 & 1.6 & $3.0 \pm 0.7$ & $28.73_{-0.1}^{0.09}$ & 32.69 & -3.95 \\
\hline 77 & 6265 & 1 & 17.3 & 1.5 & 2.02 & $13.2 \pm 1$ & $29.38_{-0.04}^{0.04}$ & 32.18 & -2.8 \\
\hline 78 & 9696 & 1.2 & 12 & 0.5 & 0.59 & $5.8 \pm 0.8$ & $29.02_{-0.06}^{0.06}$ & 34.3 & -5.28 \\
\hline 79 & 9465 & 0.2 & 13.8 & 0.8 & 0.96 & $98 \pm 2$ & $30.24_{-0.01}^{0.01}$ & 33.5 & -3.26 \\
\hline 81 & 8462 & 4.1 & 20.7 & $\ldots$ & 3.23 & $2.9 \pm 0.5$ & $28.71_{-0.08}^{0.071}$ & 31.46 & -2.75 \\
\hline 84 & 7619 & 0.8 & 14.3 & 0.9 & 1.01 & $12.6 \pm 1$ & $29.35_{-0.04}^{0.030}$ & 33.38 & -4.03 \\
\hline 87 & 8411 & 0.9 & 14 & 0.9 & 0.94 & $2.7 \pm 0.5$ & $28.68_{-0.09}^{0.07}$ & 33.56 & -4.88 \\
\hline 88 & 8689 & 6.6 & 13.9 & 1 & 1.05 & $2.1 \pm 0.5$ & $28.57_{-0.1}^{0.09}$ & 33.32 & -4.74 \\
\hline 90 & 9908 & 1.6 & 16.9 & 1.5 & 2.09 & $14.1 \pm 1$ & $29.4_{-0.03}^{0.03}$ & 32.1 & -2.7 \\
\hline 92 & 7548 & 0.6 & 20.3 & $\ldots$ & 3.18 & $1.7 \pm 0.4$ & $28.48_{-0.1}^{0.09}$ & 31.48 & -3.01 \\
\hline 94 & 9676 & 1.9 & 14.3 & 0.9 & 1.05 & $57 \pm 2$ & $30.01_{-0.01}^{0.01}$ & 33.31 & -3.3 \\
\hline 95 & 8479 & 1.1 & 19.2 & $\ldots$ & 2.62 & $2.5 \pm 0.5$ & $28.65_{-0.1}^{0.08}$ & 31.69 & -3.04 \\
\hline 97 & 12201 & 3 & 19.6 & $\ldots$ & 2.95 & $12 \pm 2$ & $29.34_{-0.07}^{0.06}$ & 31.56 & -2.22 \\
\hline 98 & 6426 & 2 & 19.8 & $\ldots$ & 2.75 & $2.0 \pm 0.5$ & $28.56_{-0.1}^{0.1}$ & 31.61 & -3.05 \\
\hline 99 & 8991 & 1.8 & 10.5 & 0.2 & 0.25 & $11.6 \pm 0.9$ & $29.32_{-0.03}^{0.03}$ & 34.73 & -5.42 \\
\hline 102 & 6322 & 3.4 & 13.2 & 0.9 & 0.94 & $5.4 \pm 1$ & $28.99_{-0.09}^{0.07}$ & 33.56 & -4.57 \\
\hline 103 & 7678 & 1.6 & 14.4 & 0.9 & 1.05 & $31.3 \pm 1$ & $29.75_{-0.02}^{0.02}$ & 33.31 & -3.56 \\
\hline 104 & 9852 & 1.1 & 12.1 & 0.7 & 0.76 & $24.7 \pm 1$ & $29.65_{-0.02}^{0.02}$ & 34 & -4.36 \\
\hline 111 & 7932 & 2 & 18.8 & 1.6 & 2.44 & $6.2 \pm 0.6$ & $29.05_{-0.05}^{0.04}$ & 31.79 & -2.74 \\
\hline 114 & 7051 & 0.8 & 20.2 & $\ldots$ & 3.26 & $7.4 \pm 0.9$ & $29.12_{-0.05}^{0.005}$ & 31.45 & -2.33 \\
\hline 115 & 6628 & 1.7 & 19.1 & $\ldots$ & 2.75 & $2.0 \pm 0.5$ & $28.55_{-0.1}^{0.1}$ & 31.62 & -3.07 \\
\hline 122 & 10017 & 0.5 & 17.4 & 1.6 & 2.16 & $9.4 \pm 0.9$ & $29.22_{-0.04}^{0.04}$ & 32.01 & -2.78 \\
\hline 125 & 8404 & 0.8 & 18.9 & $\ldots$ & 2.51 & $2.8 \pm 0.4$ & $28.7_{-0.07}^{0.06}$ & 31.74 & -3.03 \\
\hline 126 & 7782 & 0.2 & 15.8 & 1.4 & 1.71 & $58 \pm 3$ & $30.01_{-0.02}^{0.02}$ & 32.6 & -2.58 \\
\hline 127 & 10688 & 0.8 & 10 & 0.1 & 0.16 & $3.5 \pm 0.6$ & $28.79_{-0.08}^{0.07}$ & 34.92 & -6.13 \\
\hline 128 & 6970 & 2.6 & 19.8 & $\ldots$ & 2.94 & $3.4 \pm 0.6$ & $28.78_{-0.08}^{0.07}$ & 31.56 & -2.78 \\
\hline 131 & 8502 & 0.7 & 15.2 & 1.1 & 1.23 & $4.5 \pm 0.6$ & $28.9_{-0.06}^{0.06}$ & 33.05 & -4.15 \\
\hline 135 & 5576 & 1.4 & 19.5 & $\ldots$ & 2.98 & $4.0 \pm 0.7$ & $28.86_{-0.09}^{0.07}$ & 31.55 & -2.69 \\
\hline 137 & 4125 & 3.3 & 11.6 & 0.5 & 0.66 & $42 \pm 6$ & $29.87_{-0.07}^{-0.069}$ & 34.15 & -4.27 \\
\hline 139 & 7276 & 1.1 & 16.4 & 1.4 & 1.63 & $3.7 \pm 0.6$ & $28.82_{-0.07}^{0.06}$ & 32.66 & -3.84 \\
\hline 140 & 9035 & 3.2 & 18.6 & $\ldots$ & 2.63 & $6.7 \pm 0.7$ & $29.08_{-0.05}^{0.04}$ & 31.68 & -2.6 \\
\hline 142 & 6570 & 5.3 & 13.4 & 0.7 & 0.87 & $19.6 \pm 1$ & $29.55_{-0.03}^{0.03}$ & 33.77 & -4.22 \\
\hline
\end{tabular}


Table B.1. continued.

\begin{tabular}{|c|c|c|c|c|c|c|c|c|c|}
\hline$\overline{X-\text { ray Src }}$ & Opt. Id. & $\begin{array}{l}\text { Distance } \\
\text { arcsec }\end{array}$ & $\begin{array}{l}V \\
\text { mag }\end{array}$ & $\begin{array}{l}B-V \\
\text { mag }\end{array}$ & $\begin{array}{l}V-I \\
\text { mag }\end{array}$ & $\begin{array}{l}f_{\mathrm{X}}(0.3-7.9 \mathrm{keV}) \\
10^{-15} \mathrm{erg} \mathrm{s}^{-1} \mathrm{~cm}^{-2}\end{array}$ & $\begin{array}{l}\log L_{X} \\
\operatorname{erg~s}^{-1}\end{array}$ & $\begin{array}{l}\log L_{\text {bol }} \\
\operatorname{erg~s}^{-1}\end{array}$ & $\overline{\log L_{\mathrm{X}} / L_{\text {bol }}}$ \\
\hline 143 & 5066 & 3 & 18.4 & 1.6 & 2.38 & $5.8 \pm 1$ & $29.02_{-0.08}^{0.07}$ & 31.85 & -2.83 \\
\hline 144 & 7006 & 1.4 & 20.2 & $\ldots$ & 3.03 & $4.1 \pm 0.7$ & $28.87_{-0.09}^{0.07}$ & 31.53 & -2.66 \\
\hline 145 & 6473 & 2.3 & 19.4 & 1.6 & 2.72 & $2.3 \pm 0.5$ & $28.62_{-0.1}^{0.08}$ & 31.63 & -3.01 \\
\hline 146 & 10471 & 1.9 & 17.5 & 1.4 & 2.08 & $10.2 \pm 0.9$ & $29.26_{-0.04}^{0.04}$ & 32.11 & -2.85 \\
\hline 147 & 7358 & 3.3 & 17.8 & 1.5 & 2.13 & $5.3 \pm 0.8$ & $28.98_{-0.07}^{0.00}$ & 32.06 & -3.08 \\
\hline 148 & 10857 & 0.7 & 19.3 & $\ldots$ & 2.88 & $19.5 \pm 1$ & $29.54_{-0.03}^{0.03}$ & 31.58 & -2.04 \\
\hline 149 & 5901 & 2.8 & 13 & 0.7 & 0.8 & $5.5 \pm 0.9$ & $28.99_{-0.08}^{0.07}$ & 33.93 & -4.94 \\
\hline 151 & 6751 & 2 & 18.7 & 1.6 & 2.44 & $3.2 \pm 0.6$ & $28.76_{-0.1}^{0.08}$ & 31.79 & -3.04 \\
\hline 154 & 7650 & 0.6 & 12.7 & 0.6 & 0.77 & $22.1 \pm 1$ & $29.6_{-0.03}^{0.03}$ & 33.99 & -4.39 \\
\hline 155 & 11556 & 1.5 & 16.2 & 1.4 & 1.55 & $3.8 \pm 0.8$ & $28.83_{-0.1}^{0.08}$ & 32.74 & -3.91 \\
\hline 156 & 8370 & 2.2 & 20.8 & $\ldots$ & 3.16 & $1.2 \pm 0.3$ & $28.34_{-0.09}^{0.09}$ & 31.49 & -3.15 \\
\hline 159 & 9175 & 0.9 & 11.7 & 0.6 & 0.7 & $38 \pm 2$ & $29.84_{-0.02}^{0.02}$ & 34.09 & -4.25 \\
\hline 163 & 6605 & 2.3 & 13.6 & 0.8 & 0.96 & $17.8 \pm 1$ & $29.5_{-0.03}^{0.03}$ & 33.51 & -4.01 \\
\hline 164 & 5261 & 5.5 & 15.1 & 1.1 & 1.22 & $7.3 \pm 1$ & $29.11_{-0.07}^{0.06}$ & 33.08 & -3.96 \\
\hline 166 & 8192 & 2.8 & 13.9 & 0.9 & 0.95 & $6.5 \pm 0.6$ & $29.06_{-0.04}^{0.04}$ & 33.54 & -4.47 \\
\hline 171 & 6373 & 1 & 11.3 & 0.5 & 0.66 & $17.1 \pm 1$ & $29.49_{-0.03}^{0.03}$ & 34.15 & -4.66 \\
\hline 174 & 8172 & 0.4 & 12 & 0.5 & 0.68 & $4.4 \pm 0.5$ & $28.9_{-0.05}^{0.05}$ & 34.1 & -5.21 \\
\hline 175 & 12005 & 1.6 & 11.6 & 0.6 & 0.62 & $9.9 \pm 1$ & $29.25_{-0.06}^{-0.05}$ & 34.24 & -4.99 \\
\hline 178 & 9048 & 1.6 & 10.8 & 0.3 & 0.36 & $2.2 \pm 0.4$ & $28.6_{-0.09}^{0.07}$ & 34.58 & -5.98 \\
\hline 180 & 11713 & 1.9 & 12.4 & 0.7 & 0.8 & $4.7 \pm 0.8$ & $28.93_{-0.08}^{0.07}$ & 33.93 & -5 \\
\hline 183 & 5899 & 2.6 & 18.4 & 1.5 & 2.37 & $7.5 \pm 0.9$ & $29.13_{-0.05}^{0.05}$ & 31.85 & -2.72 \\
\hline 184 & 11348 & 0.6 & 19.2 & $\ldots$ & 2.61 & $4.3 \pm 0.7$ & $28.89_{-0.07}^{0.06}$ & 31.69 & -2.8 \\
\hline 185 & 8893 & 1.2 & 16.2 & 1.4 & 1.82 & $37.5 \pm 1$ & $29.83_{-0.02}^{0.02}$ & 32.47 & -2.64 \\
\hline 187 & 10753 & 1.5 & 13.7 & 0.8 & 0.9 & $2.1 \pm 0.5$ & $28.58_{-0.1}^{0.1}$ & 33.71 & -5.13 \\
\hline 190 & 9025 & 0.6 & 17.5 & 1.6 & 1.97 & $3.9 \pm 0.5$ & $28.84_{-0.06}^{0.05}$ & 32.22 & -3.38 \\
\hline 191 & 11233 & 1.9 & 13.9 & 0.8 & 1.02 & $80 \pm 2$ & $30.16_{-0.01}^{0.01}$ & 33.36 & -3.2 \\
\hline 194 & 7231 & 3.2 & 11.2 & 0.4 & 0.49 & $5.3 \pm 0.7$ & $28.98_{-0.06}^{0.05}$ & 34.44 & -5.46 \\
\hline 197 & 6649 & 0.9 & 14 & 0.8 & 0.98 & $41.6 \pm 2$ & $29.87_{-0.02}^{-0.02}$ & 33.45 & -3.58 \\
\hline 198 & 8647 & 1.7 & 20 & $\ldots$ & 3.11 & $2.2 \pm 0.4$ & $28.6_{-0.09}^{0.07}$ & 31.51 & -2.9 \\
\hline 199 & 7585 & 0.8 & 12.9 & 0.7 & 0.88 & $46 \pm 2$ & $29.91_{-0.02}^{0.01}$ & 33.76 & -3.84 \\
\hline 200 & 8036 & 1.8 & 17.6 & $\ldots$ & 2.41 & $16.1 \pm 0.9$ & $29.46_{-0.03}^{0.02}$ & 31.81 & -2.35 \\
\hline 201 & 9283 & 0.6 & 16.9 & 1.4 & 1.84 & $2.2 \pm 0.4$ & $28.59_{-0.08}^{0.070}$ & 32.45 & -3.87 \\
\hline 203 & 6852 & 1.2 & 13.7 & 0.8 & 0.91 & $5.7 \pm 0.6$ & $29.01_{-0.05}^{0.050}$ & 33.68 & -4.67 \\
\hline 204 & 5179 & 1.1 & 19.6 & $\ldots$ & 2.78 & $5.2 \pm 1$ & $28.97_{-0.09}^{0.07}$ & 31.61 & -2.64 \\
\hline 205 & 9185 & 1 & 18.3 & 1.5 & 2.27 & $8.0 \pm 0.7$ & $29.16_{-0.04}^{0.04}$ & 31.93 & -2.77 \\
\hline 206 & 4598 & 0.5 & 14 & 0.9 & 1.02 & $120 \pm 5$ & $30.33_{-0.02}^{0.02}$ & 33.37 & -3.04 \\
\hline 207 & 5833 & 1.2 & 12.4 & 0.6 & 0.72 & $7.9 \pm 0.8$ & $29.15_{-0.05}^{0.04}$ & 34.06 & -4.91 \\
\hline 208 & 10452 & 1.2 & 19.7 & $\ldots$ & 2.97 & $4.1 \pm 0.6$ & $28.87_{-0.07}^{-0.06}$ & 31.55 & -2.68 \\
\hline 209 & 11082 & 2.6 & 9.6 & 0.1 & 0.08 & $18.7 \pm 1$ & $29.53_{-0.03}^{0.03}$ & 35.3 & -5.78 \\
\hline $2-10$ & 10210 & 1.3 & 10.8 & 0.3 & 0.33 & $4.7 \pm 0.6$ & $28.92_{-0.06}^{0.05}$ & 34.62 & -5.7 \\
\hline 211 & 9703 & 1.4 & 18.5 & 1.6 & 2.48 & $5.9 \pm 0.6$ & $29.02_{-0.04}^{0.04}$ & 31.75 & -2.73 \\
\hline 213 & 7962 & 1 & 12 & 0.5 & 0.65 & $3.9 \pm 0.5$ & $28.85_{-0.06}^{0.005}$ & 34.18 & -5.33 \\
\hline 214 & 10702 & 1 & 16.3 & 1.5 & 1.85 & $5.7 \pm 0.7$ & $29.01_{-0.06}^{0.050}$ & 32.43 & -3.43 \\
\hline 218 & 11372 & 1.1 & 18.4 & 1.6 & 2.33 & $1.7 \pm 0.4$ & $28.49_{-0.1}^{0.09}$ & 31.88 & -3.39 \\
\hline 219 & 8932 & 1.9 & 12.9 & 0.8 & 0.85 & $3.3 \pm 0.5$ & $28.78_{-0.07}^{0.06}$ & 33.82 & -5.05 \\
\hline 220 & 10010 & 1.4 & 17.2 & 1.5 & 1.93 & $7.9 \pm 0.7$ & $29.15_{-0.04}^{0.04}$ & 32.31 & -3.15 \\
\hline 222 & 6640 & 1.1 & 17.4 & 1.5 & 2.08 & $2.8 \pm 0.5$ & $28.7_{-0.08}^{0.06}$ & 32.11 & -3.41 \\
\hline 223 & 5280 & 0.8 & 17.3 & 1.6 & 2.15 & $10.1 \pm 1$ & $29.26_{-0.06}^{-0.05}$ & 32.03 & -2.77 \\
\hline 225 & 6106 & 2.7 & 12 & 0.5 & 0.65 & $9.2 \pm 1$ & $29.22_{-0.05}^{0.04}$ & 34.16 & -4.94 \\
\hline 228 & 5446 & 1.3 & 9.7 & 0.1 & 0.2 & $18.6 \pm 1$ & $29.52_{-0.03}^{0.003}$ & 34.93 & -5.41 \\
\hline 229 & 7483 & 2.8 & 13.1 & 0.8 & 0.88 & $2 \pm 0.4$ & $28.55_{-0.1}^{0.09}$ & 33.75 & -5.19 \\
\hline 230 & 9140 & 1.3 & 13.4 & 0.7 & 0.84 & $45 \pm 2$ & $29.9_{-0.02}^{0.02 .1}$ & 33.85 & -3.95 \\
\hline 231 & 8408 & 1.6 & 15.2 & 1.1 & 1.28 & $8.4 \pm 0.7$ & $29.18_{-0.04}^{0.03}$ & 32.99 & -3.82 \\
\hline 233 & 8654 & 0.8 & 14.8 & 1.1 & 1.1 & $3.0 \pm 0.5$ & $28.73_{-0.07}^{0.064}$ & 33.2 & -4.46 \\
\hline 234 & 10871 & 2.1 & 14.5 & 1 & 1.06 & $7.1 \pm 0.8$ & $29.11_{-0.05}^{0.05}$ & 33.29 & -4.18 \\
\hline 235 & 6452 & 0.4 & 13 & 0.7 & 0.79 & $10.1 \pm 1$ & $29.26_{-0.04}^{0.004}$ & 33.94 & -4.68 \\
\hline 236 & 7864 & 0.6 & 12.1 & 0.6 & 0.74 & $43.5 \pm 1$ & $29.89_{-0.01}^{0.01}$ & 34.03 & -4.14 \\
\hline 238 & 8969 & 1.6 & 18.9 & $\ldots$ & 2.49 & $1.6 \pm 0.3$ & $28.47_{-0.1}^{0.08}$ & 31.75 & -3.28 \\
\hline 240 & 6263 & 0.8 & 14.2 & 0.9 & 1.01 & $3.2 \pm 0.5$ & $28.75_{-0.08}^{0.06}$ & 33.38 & -4.63 \\
\hline
\end{tabular}


Table B.1. continued.

\begin{tabular}{|c|c|c|c|c|c|c|c|c|c|}
\hline$\overline{\mathrm{X} \text {-ray Src }}$ & Opt. Id. & $\begin{array}{l}\text { Distance } \\
\text { arcsec }\end{array}$ & $\begin{array}{l}V \\
\text { mag }\end{array}$ & $\begin{array}{l}B-V \\
\text { mag }\end{array}$ & $\begin{array}{l}V-I \\
\text { mag }\end{array}$ & $\begin{array}{l}f_{\mathrm{X}}(0.3-7.9 \mathrm{keV}) \\
10^{-15} \mathrm{erg} \mathrm{s}^{-1} \mathrm{~cm}^{-2}\end{array}$ & $\begin{array}{l}\log L_{X} \\
\operatorname{erg~s}^{-1}\end{array}$ & $\begin{array}{l}\log L_{\text {bol }} \\
\operatorname{erg~s}^{-1}\end{array}$ & $\overline{l \log L_{\mathrm{X}} / L_{\text {bol }}}$ \\
\hline 242 & 7001 & 0.7 & 15.7 & 1.3 & 1.6 & $10.5 \pm 0.8$ & $29.27_{-0.03}^{0.03}$ & 32.69 & -3.41 \\
\hline 245 & 5061 & 2.4 & 15.4 & 1.3 & 1.54 & $39 \pm 2$ & $29.84_{-0.02}^{0.02}$ & 32.75 & -2.91 \\
\hline 246 & 9202 & 1 & 15.2 & 1.1 & 1.24 & $4.4 \pm 0.5$ & $28.9_{-0.06}^{0.05}$ & 33.04 & -4.15 \\
\hline 249 & 8556 & 4 & 18.9 & 1.5 & 2.57 & $3.9 \pm 0.6$ & $28.84_{-0.07}^{0.06}$ & 31.71 & -2.87 \\
\hline 250 & 12728 & 2.6 & 16.2 & 1.3 & 1.58 & $17 \pm 2$ & $29.5_{-0.06}^{0.06}$ & 32.7 & -3.21 \\
\hline 251 & 10348 & 1.8 & 20 & $\ldots$ & 3 & $3.8 \pm 0.6$ & $28.83_{-0.07}^{0.06}$ & 31.54 & -2.71 \\
\hline 252 & 7959 & 0.6 & 14 & 1 & 1.12 & $17.0 \pm 0.9$ & $29.48_{-0.02}^{0.02}$ & 33.17 & -3.69 \\
\hline 254 & 7505 & 1.3 & 19.7 & $\ldots$ & 2.87 & $13.3 \pm 1$ & $29.38_{-0.03}^{0.03}$ & 31.58 & -2.21 \\
\hline 255 & 6603 & 1.2 & 18.1 & 1.6 & 2.36 & $6.0 \pm 0.7$ & $29.03_{-0.06}^{0.05}$ & 31.86 & -2.83 \\
\hline 256 & 10929 & 0.5 & 19.8 & $\ldots$ & 2.75 & $1.5 \pm 0.4$ & $28.43_{-0.1}^{0.100}$ & 31.62 & -3.18 \\
\hline 257 & 10863 & 3.5 & 12.3 & 0.6 & 0.7 & $18.9 \pm 1$ & $29.53_{-0.03}^{0.03}$ & 34.09 & -4.56 \\
\hline 258 & 4560 & 4.2 & 12.4 & 0.7 & 0.8 & $12.1 \pm 2$ & $29.34_{-0.07}^{0.06}$ & 33.94 & -4.6 \\
\hline 259 & 8997 & 1.1 & 14.6 & 1 & 1.14 & $34.8 \pm 1$ & $29.79_{-0.02}^{0.02}$ & 33.16 & -3.36 \\
\hline 260 & 7690 & 2.5 & 15.2 & 1.3 & 1.42 & $7.1 \pm 0.7$ & $29.1_{-0.04}^{0.04}$ & 32.87 & -3.76 \\
\hline 262 & 6492 & 0.7 & 15.9 & 1.3 & 1.5 & $9.8 \pm 0.9$ & $29.25_{-0.04}^{0.04}$ & 32.8 & -3.55 \\
\hline 263 & 9512 & 0.9 & 17.9 & 1.6 & 2.26 & $7.3 \pm 0.7$ & $29.12_{-0.04}^{0.04}$ & 31.93 & -2.81 \\
\hline 269 & 10894 & 1.9 & 11.9 & 0.6 & -10 & $20.0 \pm 1$ & $29.55_{-0.03}^{0.03}$ & 33.97 & -4.42 \\
\hline 271 & 9286 & 0.2 & 14.2 & 0.9 & 1 & $2.7 \pm 0.5$ & $28.68_{-0.08}^{0.07}$ & 33.39 & -4.71 \\
\hline 272 & 12302 & 0.3 & 12 & 0.5 & 0.65 & $21 \pm 2$ & $29.57_{-0.05}^{-0.04}$ & 34.17 & -4.6 \\
\hline 273 & 10754 & 2.3 & 17.8 & 1.6 & 2.38 & $9.7 \pm 0.9$ & $29.24_{-0.04}^{0.04}$ & 31.84 & -2.6 \\
\hline 274 & 12649 & 1.8 & 12.4 & 0.6 & 0.74 & $26 \pm 3$ & $29.67_{-0.05}^{0.04}$ & 34.03 & -4.36 \\
\hline 275 & 8458 & 1.5 & 11.5 & 0.5 & 0.66 & $33.3 \pm 1$ & $29.78_{-0.02}^{0.01}$ & 34.13 & -4.36 \\
\hline 276 & 8776 & 1.2 & 15.8 & 1.3 & 1.48 & $5.7 \pm 0.6$ & $29.01_{-0.05}^{0.04}$ & 32.82 & -3.81 \\
\hline 278 & 7120 & 2.8 & 19.6 & $\ldots$ & 2.64 & $2.3 \pm 0.5$ & $28.62_{-0.1}^{0.08}$ & 31.67 & -3.05 \\
\hline 279 & 7553 & 1.9 & 13.8 & 0.8 & 0.92 & $9.7 \pm 0.8$ & $29.24_{-0.04}^{0.03}$ & 33.63 & -4.39 \\
\hline 282 & 5989 & 0.7 & 20.2 & $\ldots$ & 2.97 & $8.8 \pm 1$ & $29.2_{-0.05}^{0.05}$ & 31.55 & -2.36 \\
\hline 283 & 7967 & 2.5 & 12.7 & 0.6 & 0.74 & $6.1 \pm 0.6$ & $29.04_{-0.04}^{0.04}$ & 34.02 & -4.98 \\
\hline 285 & 11718 & 2.9 & 17.4 & 1.5 & 2.04 & $12.5 \pm 1$ & $29.35_{-0.05}^{-0.04}$ & 32.16 & -2.81 \\
\hline 289 & 9061 & 1.5 & 17.5 & 1.7 & 1.99 & $2.0 \pm 0.4$ & $28.56_{-0.1}^{0.08}$ & 32.21 & -3.64 \\
\hline 290 & 8099 & 0.2 & 12.2 & 0.6 & 0.68 & $28.5 \pm 1$ & $29.71_{-0.02}^{0.02}$ & 34.11 & -4.4 \\
\hline 295 & 9054 & 3.3 & 12.8 & 0.7 & 0.84 & $42.0 \pm 1$ & $29.88_{-0.01}^{0.01}$ & 33.85 & -3.98 \\
\hline 298 & 7104 & 1 & 12.7 & 0.6 & 0.75 & $11.9 \pm 0.9$ & $29.33_{-0.03}^{0.03}$ & 34.01 & -4.69 \\
\hline 300 & 7667 & 0.8 & 9.9 & 0.2 & 0.25 & $25.2 \pm 1$ & $29.65_{-0.02}^{0.02}$ & 34.8 & -5.15 \\
\hline 302 & 8737 & 0.8 & 14.8 & 1 & 1.12 & $1.9 \pm 0.4$ & $28.54_{-0.09}^{0.08}$ & 33.18 & -4.63 \\
\hline 304 & 10143 & 1.8 & 19.9 & $\ldots$ & 3.01 & $3.5 \pm 0.6$ & $28.8_{-0.08}^{0.07}$ & 31.54 & -2.74 \\
\hline 305 & 6268 & 0.3 & 14.9 & 1.1 & 1.2 & $18.5 \pm 1$ & $29.52_{-0.03}^{0.03}$ & 33.09 & -3.57 \\
\hline 310 & 11782 & 1.6 & 14.6 & 1 & 1.16 & $1.9 \pm 0.7$ & $28.53_{-0.2}^{0.103}$ & 33.14 & -4.61 \\
\hline 311 & 8332 & 1.1 & 9.7 & 0.2 & 0.28 & $8.9 \pm 0.7$ & $29.2_{-0.04}^{0.03}$ & 34.76 & -5.56 \\
\hline 312 & 10191 & 2 & 19.4 & $\ldots$ & 3.06 & $2.7 \pm 0.5$ & $28.68_{-0.08}^{0.07}$ & 31.52 & -2.84 \\
\hline 313 & 5024 & 0.6 & 16.3 & 1.3 & 1.66 & $18 \pm 2$ & $29.5_{-0.04}^{0.04}$ & 32.64 & -3.14 \\
\hline 315 & 6847 & 3.4 & 18.9 & $\ldots$ & 2.43 & $2.8 \pm 0.6$ & $28.7_{-0.1}^{0.08}$ & 31.8 & -3.1 \\
\hline 320 & 11451 & 1.8 & 19.4 & $\ldots$ & 2.68 & $2.4 \pm 0.6$ & $28.63_{-0.1}^{0.1}$ & 31.65 & -3.02 \\
\hline 322 & 7203 & 1.9 & 19.6 & $\ldots$ & 2.62 & $4.9 \pm 0.7$ & $28.94_{-0.06}^{0.05}$ & 31.69 & -2.74 \\
\hline 323 & 10046 & 1.3 & 14.8 & 1 & 1.14 & $46.8 \pm 1$ & $29.92_{-0.01}^{0.01}$ & 33.16 & -3.23 \\
\hline 327 & 12874 & 1.7 & 13.5 & 0.9 & 1.05 & $29 \pm 5$ & $29.71_{-0.08}^{0.07}$ & 33.31 & -3.6 \\
\hline 328 & 11153 & 1.5 & 20.1 & $\ldots$ & 2.8 & $5.8 \pm 0.8$ & $29.02_{-0.07}^{-0.06}$ & 31.6 & -2.59 \\
\hline 331 & 11485 & 1 & 14.8 & 1 & 1.21 & $25 \pm 2$ & $29.65_{-0.03}^{0.03}$ & 33.09 & -3.44 \\
\hline 332 & 7622 & 6.9 & 17.2 & 1.5 & 1.91 & $16.1 \pm 1$ & $29.46_{-0.03}^{0.03}$ & 32.35 & -2.89 \\
\hline 337 & 7420 & 2.3 & 17.8 & 1.6 & 2.12 & $4.7 \pm 0.6$ & $28.92_{-0.06}^{0.06}$ & 32.07 & -3.15 \\
\hline 338 & 6508 & 2 & 18.4 & 1.6 & 2.6 & $5.9 \pm 0.9$ & $29.03_{-0.07}^{0.06}$ & 31.7 & -2.67 \\
\hline 339 & 6676 & 1 & 14.3 & 0.9 & 1.01 & $2.4 \pm 0.5$ & $28.64_{-0.1}^{0.08}$ & 33.38 & -4.74 \\
\hline 342 & 11970 & 2 & 10.8 & 0.3 & 0.4 & $28 \pm 2$ & $29.7_{-0.03}^{0.03}$ & 34.53 & -4.83 \\
\hline 343 & 8529 & 1 & 12.3 & 0.6 & 0.71 & $31.8 \pm 1$ & $29.76_{-0.02}^{0.02}$ & 34.07 & -4.32 \\
\hline 346 & 8886 & 4.3 & 9.7 & 0.1 & 0.13 & $55.2 \pm 2$ & $30_{-0.02}^{0.02^{-c}}$ & 35.08 & -5.08 \\
\hline 347 & 9328 & 4.3 & 12.2 & 0.6 & 0.67 & $18.7 \pm 1$ & $29.52_{-0.03}^{0.03}$ & 34.12 & -4.59 \\
\hline 349 & 9572 & 0.6 & 19.1 & $\ldots$ & 2.87 & $2.0 \pm 0.4$ & $28.56_{-0.09}^{0.08}$ & 31.58 & -3.02 \\
\hline 350 & 8536 & 0.9 & 12.9 & 0.7 & 0.76 & $3.0 \pm 0.5$ & $28.72_{-0.09}^{0.07}$ & 34 & -5.27 \\
\hline 351 & 10330 & 0.4 & 19.6 & $\ldots$ & 2.72 & $2.5 \pm 0.5$ & $28.65_{-0.1}^{0.09}$ & 31.63 & -2.98 \\
\hline 353 & 10695 & 0.9 & 16.8 & 1.5 & 1.78 & $7.9 \pm 1$ & $29.15_{-0.06}^{0.05}$ & 32.52 & -3.37 \\
\hline
\end{tabular}


Table B.1. continued.

\begin{tabular}{|c|c|c|c|c|c|c|c|c|c|}
\hline$\overline{X-\text { ray Src }}$ & Opt. Id. & $\begin{array}{l}\text { Distance } \\
\text { arcsec }\end{array}$ & $\begin{array}{l}V \\
\text { mag }\end{array}$ & $\begin{array}{l}B-V \\
\text { mag }\end{array}$ & $\begin{array}{l}V-I \\
\text { mag }\end{array}$ & $\begin{array}{l}f_{\mathrm{X}}(0.3-7.9 \mathrm{keV}) \\
10^{-15} \mathrm{erg} \mathrm{s}^{-1} \mathrm{~cm}^{-2}\end{array}$ & $\begin{array}{l}\log L_{X} \\
\operatorname{erg~s}^{-1}\end{array}$ & $\begin{array}{l}\log L_{\text {bol }} \\
\operatorname{erg~s}^{-1}\end{array}$ & $\overline{\log L_{\mathrm{X}} / L_{\text {bol }}}$ \\
\hline 355 & 11867 & 3.7 & 12.6 & 0.6 & 0.73 & $7.6 \pm 1$ & $29.13_{-0.08}^{0.07}$ & 34.05 & -4.91 \\
\hline 358 & 7322 & 3.4 & 15 & 1.1 & 1.14 & $7.0 \pm 0.9$ & $29.1_{-0.06}^{0.05}$ & 33.16 & -4.07 \\
\hline 360 & 10047 & 1.9 & 18.5 & 1.7 & 2.34 & $5.3 \pm 0.7$ & $28.98_{-0.06}^{0.00}$ & 31.87 & -2.89 \\
\hline 362 & 5836 & 1.4 & 19.6 & $\ldots$ & 3.07 & $4.6 \pm 0.9$ & $28.92_{-0.09}^{0.070}$ & 31.52 & -2.6 \\
\hline 363 & 6029 & 0.7 & 12.2 & 0.7 & 0.77 & $49 \pm 2$ & $29.94_{-0.02}^{0.02}$ & 33.98 & -4.04 \\
\hline 364 & 6180 & 2.2 & 18.6 & 1.6 & 2.71 & $4.7 \pm 0.7$ & $28.93_{-0.07}^{0.06}$ & 31.64 & -2.71 \\
\hline 367 & 7595 & 1 & 12.6 & 0.6 & 0.7 & $12.4 \pm 0.9$ & $29.35_{-0.03}^{0.03}$ & 34.08 & -4.74 \\
\hline 370 & 10605 & 1.7 & 13.6 & 0.8 & 0.89 & $4.5 \pm 0.7$ & $28.9_{-0.07}^{0.06}$ & 33.73 & -4.83 \\
\hline 371 & 9446 & 2.4 & 13.6 & 0.8 & 0.85 & $5.0 \pm 0.9$ & $28.95_{-0.08}^{0.07}$ & 33.82 & -4.87 \\
\hline 372 & 8634 & 1 & 14.5 & 0.9 & 1.12 & $58 \pm 2$ & $30.02_{-0.01}^{0.001}$ & 33.18 & -3.16 \\
\hline 375 & 12118 & 2 & 14.3 & 0.9 & 1.08 & $9 \pm 2$ & $29.19_{-0.1}^{0.08}$ & 33.24 & -4.05 \\
\hline 384 & 11132 & 1.2 & 17.4 & 1.5 & 2.01 & $12.2 \pm 1$ & $29.34_{-0.05}^{0.05}$ & 32.19 & -2.85 \\
\hline 387 & 5312 & 4.3 & 18 & 1.5 & 2.24 & $6.7 \pm 1$ & $29.08_{-0.1}^{0.08}$ & 31.94 & -2.86 \\
\hline 388 & 9453 & 0.8 & 17.7 & 1.6 & 2.1 & $4.7 \pm 0.7$ & $28.93_{-0.07}^{0.06}$ & 32.1 & -3.17 \\
\hline 390 & 9241 & 1.8 & 9.6 & 0.1 & 0.08 & $4.0 \pm 0.7$ & $28.86_{-0.09}^{0.07}$ & 35.17 & -6.32 \\
\hline 391 & 6577 & 2.1 & 19.4 & $\ldots$ & 2.66 & $3.5 \pm 0.7$ & $28.8_{-0.1}^{0.08}$ & 31.66 & -2.86 \\
\hline 396 & 6070 & 2.7 & 15 & 1.1 & 1.2 & $19 \pm 2$ & $29.53_{-0.04}^{0.04}$ & 33.09 & -3.56 \\
\hline 398 & 8261 & 2.8 & 12 & 0.5 & 0.6 & $16.1 \pm 1$ & $29.46_{-0.03}^{0.03}$ & 34.28 & -4.82 \\
\hline 400 & 7028 & 1.7 & 18.7 & 1.6 & 2.46 & $4.8 \pm 0.9$ & $28.94_{-0.09}^{0.08}$ & 31.77 & -2.83 \\
\hline 402 & 6650 & 1.1 & 18.1 & 1.7 & 2.18 & $13.1 \pm 1$ & $29.37_{-0.05}^{0.04}$ & 31.98 & -2.61 \\
\hline 403 & 7918 & 2.1 & 18.8 & 1.6 & 2.39 & $7.9 \pm 1$ & $29.15_{-0.06}^{0.05}$ & 31.84 & -2.69 \\
\hline 404 & 10884 & 2 & 19.1 & 1.6 & 2.65 & $4.2 \pm 0.8$ & $28.87_{-0.1}^{0.08}$ & 31.67 & -2.8 \\
\hline 405 & 9079 & 6.5 & 20.3 & $\ldots$ & 3 & $5.3 \pm 0.8$ & $28.97_{-0.07}^{-0.06}$ & 31.55 & -2.57 \\
\hline 408 & 8242 & 4.3 & 17.3 & 1.5 & 1.89 & $2.0 \pm 0.5$ & $28.55_{-0.1}^{0.1}$ & 32.37 & -3.83 \\
\hline 409 & 8935 & 0.8 & 10.6 & 0.3 & 0.39 & $16.7 \pm 1$ & $29.48_{-0.04}^{0.03}$ & 34.56 & -5.08 \\
\hline 412 & 11982 & 1.9 & 19.1 & $\ldots$ & 2.56 & $5.1 \pm 1$ & $28.96_{-0.1}^{0.1}$ & 31.72 & -2.76 \\
\hline 414 & 8660 & 1.9 & 12.4 & 0.6 & 0.67 & $27 \pm 2$ & $29.69_{-0.03}^{0.02}$ & 34.12 & -4.43 \\
\hline 416 & 9727 & 5.6 & 19.8 & $\ldots$ & 2.9 & $5.1 \pm 1$ & $28.96_{-0.1}^{0.08}$ & 31.57 & -2.62 \\
\hline 417 & 8989 & 1.8 & 18.6 & 1.5 & 2.42 & $3.8 \pm 0.8$ & $28.83_{-0.1}^{0.08}$ & 31.81 & -2.98 \\
\hline 418 & 10902 & 1.7 & 18.1 & 1.6 & 2.25 & $5.6 \pm 1$ & $29_{-0.09}^{0.08}$ & 31.94 & -2.94 \\
\hline 419 & 10293 & 0.5 & 15.5 & 1.2 & 1.31 & $5.5 \pm 0.9$ & $28.99_{-0.08}^{0.07}$ & 32.95 & -3.96 \\
\hline 425 & 10040 & 3.3 & 14.9 & 1 & 1.17 & $40 \pm 3$ & $29.86_{-0.03}^{0.03}$ & 33.13 & -3.27 \\
\hline 426 & 9882 & 2.2 & 9.8 & 0.2 & 0.24 & $13.0 \pm 0.8$ & $29.37_{-0.03}^{0.03}$ & 34.83 & -5.47 \\
\hline 428 & 7596 & 6.8 & 16.2 & 1.4 & 1.65 & $19 \pm 2$ & $29.54_{-0.04}^{0.04}$ & 32.65 & -3.11 \\
\hline 432 & 8453 & 3.1 & 15.9 & 1.3 & 1.47 & $3.9 \pm 0.9$ & $28.85_{-0.1}^{0.09}$ & 32.82 & -3.97 \\
\hline 434 & 9835 & 1 & 14.8 & 1 & 1.13 & $51 \pm 2$ & $29.96_{-0.02}^{0.02}$ & 33.17 & -3.21 \\
\hline 436 & 8397 & 1 & 20 & $\ldots$ & 3.08 & $2.9 \pm 0.7$ & $28.71_{-0.1}^{0.09}$ & 31.52 & -2.81 \\
\hline 437 & 7941 & 1.1 & 13.1 & 0.7 & 0.77 & $8.0 \pm 1$ & $29.15_{-0.07}^{0.06}$ & 33.98 & -4.83 \\
\hline 439 & 10817 & 0.7 & 11.9 & 0.5 & 0.65 & $375 \pm 10$ & $30.83_{-0.02}^{0.02}$ & 34.16 & -3.33 \\
\hline 442 & 10957 & 3.4 & 17.7 & 1.6 & 2.31 & $4.8 \pm 1$ & $28.93_{-0.1}^{0.09}$ & 31.9 & -2.97 \\
\hline 446 & 8999 & 0.8 & 13.4 & 0.7 & 0.85 & $4.6 \pm 1$ & $28.92_{-0.1}^{0.09}$ & 33.82 & -4.9 \\
\hline 453 & 5957 & 5 & 14.6 & 1 & 1.08 & $41 \pm 6$ & $29.87_{-0.07}^{0.06}$ & 33.25 & -3.39 \\
\hline 455 & 10434 & 2.5 & 18.1 & 1.6 & 2.29 & $6.9 \pm 1$ & $29.09_{-0.1}^{0.09}$ & 31.91 & -2.82 \\
\hline 456 & 9486 & 0.4 & 12.6 & 0.8 & 0.86 & $39 \pm 3$ & $29.85_{-0.03}^{0.03}$ & 33.79 & -3.94 \\
\hline 4 & 8665 & 1.9 & 13.1 & 0.7 & 0.83 & $7 \pm 1$ & $29.1_{-0.09}^{0.07}$ & 33.87 & -4.77 \\
\hline 4 & 8675 & 5.1 & 13.2 & $\ldots$ & 0.84 & $7 \pm 1$ & $29.1_{-0.09}^{0.079}$ & 33.86 & -4.75 \\
\hline 36 & 4750 & 0.6 & 12.3 & 0.5 & 0.59 & $9 \pm 2$ & $29.2_{-0.1}^{0.08}$ & 34.29 & -5.09 \\
\hline 36 & 4760 & 3.4 & 13.1 & 0.7 & 0.83 & $9 \pm 2$ & $29.2_{-0.1}^{0.08}$ & 33.86 & -4.66 \\
\hline 85 & 8641 & 3.1 & 14.1 & 1.1 & 1.14 & $34.0 \pm 1$ & $29.78_{-0.01}^{0.01}$ & 33.16 & -3.37 \\
\hline 85 & 8645 & 1.4 & 14.1 & 1.1 & 1.14 & $34.0 \pm 1$ & $29.78_{-0.01}^{0.001}$ & 33.16 & -3.37 \\
\hline 105 & 8967 & 1.5 & 14.8 & 1 & 1.14 & $36 \pm 2$ & $29.81_{-0.02}^{0.02}$ & 33.16 & -3.34 \\
\hline 303 & 5835 & 4.1 & 13.3 & $\ldots$ & 0.84 & $5.8 \pm 0.6$ & $29.02_{-0.04}^{0.002}$ & 33.85 & -4.83 \\
\hline 303 & 5862 & 1.4 & 13.3 & 0.7 & 0.84 & $5.8 \pm 0.6$ & $29.02_{-0.04}^{0.04}$ & 33.84 & -4.82 \\
\hline 385 & 5887 & 2.2 & 13.7 & 0.9 & 0.99 & $9.8 \pm 1$ & $29.25_{-0.06}^{0.054}$ & 33.42 & -4.17 \\
\hline 186 & 15496 & 2 & 8.4 & 0 & $\ldots$ & $31 \pm 2$ & $30.07_{-0.01}^{0.01}$ & 36.35 & -6.28 \\
\hline 193 & 15499 & 2.5 & 9.3 & 0.1 & $\ldots$ & $16.9 \pm 1$ & $29.48_{-0.03}^{0.03}$ & 35.03 & -5.55 \\
\hline 89 & 15500 & 3.3 & 8.8 & 0.1 & $\ldots$ & $18.6 \pm 1$ & $29.52_{-0.03}^{0.03}$ & 35.45 & -5.92 \\
\hline 423 & 15501 & 0.5 & 8.4 & 0 & $\ldots$ & $18 \pm 2$ & $29.51_{-0.05}^{0.04}$ & 36.75 & -7.24 \\
\hline 192 & 15503 & 1.3 & 8.9 & 0.1 & $\ldots$ & $8.6 \pm 0.8$ & $29.19_{-0.04}^{0.04}$ & 35.27 & -6.08 \\
\hline
\end{tabular}


I. Pillitteri et al.: Deep X-ray survey of NGC 2516 with XMM-Newton, Online Material p 16

Table B.1. continued.

\begin{tabular}{|c|c|c|c|c|c|c|c|c|c|}
\hline X-ray Src & $\overline{\text { Opt. Id. }}$ & $\begin{array}{l}\text { Distance } \\
\text { arcsec }\end{array}$ & $\begin{array}{l}V \\
\text { mag }\end{array}$ & $\begin{array}{l}B-V \\
\text { mag }\end{array}$ & $\begin{array}{l}V-I \\
\text { mag }\end{array}$ & $\begin{array}{l}f_{\mathrm{X}}(0.3-7.9 \mathrm{keV}) \\
10^{-15} \mathrm{erg} \mathrm{s}^{-1} \mathrm{~cm}^{-2}\end{array}$ & $\begin{array}{l}\log L_{\mathrm{X}} \\
\mathrm{erg} \mathrm{s}^{-1}\end{array}$ & $\begin{array}{l}\log L_{\text {bol }} \\
\mathrm{erg} \mathrm{s}^{-1}\end{array}$ & $\overline{\log L_{\mathrm{X}} / L_{\mathrm{bol}}}$ \\
\hline 132 & 15504 & 0.6 & 9.5 & 0.2 & $\ldots$ & $19.6 \pm 1$ & $29.55_{-0.02}^{0.02}$ & 34.77 & -5.22 \\
\hline 141 & 15506 & 1.9 & 8 & 0 & $\ldots$ & $29.5 \pm 1$ & $29.67_{-0.03}^{0.02}$ & 36.9 & -7.23 \\
\hline 141 & 15507 & 3.9 & 7.2 & 0 & $\ldots$ & $29.5 \pm 1$ & $29.67_{-0.03}^{0.02}$ & 36.45 & -6.78 \\
\hline 176 & 15509 & 1.7 & 5.8 & -0.1 & $\ldots$ & $102 \pm 2$ & $29.63_{-0.05}^{0.04}$ & $\ldots$ & $\ldots$ \\
\hline 399 & 15510 & 4.7 & 9.5 & 0.1 & $\ldots$ & $131 \pm 4$ & $30.37_{-0.01}^{0.001}$ & 35.27 & -4.9 \\
\hline 188 & 15512 & 0.9 & 9.1 & 0 & $\ldots$ & $41 \pm 2$ & $30.61_{-0.004}^{0.004}$ & 36.21 & -5.6 \\
\hline 232 & 15540 & 0.6 & 8.4 & 0.1 & $\ldots$ & $4.2 \pm 0.6$ & $28.88_{-0.06}^{0.06}$ & 35.45 & -6.57 \\
\hline
\end{tabular}


I. Pillitteri et al.: Deep X-ray survey of NGC 2516 with XMM-Newton, Online Material p 17

Appendix C: X-ray upper limits of NGC 2516 stars 
I. Pillitteri et al.: Deep X-ray survey of NGC 2516 with XMM-Newton, Online Material p 18

Table C.1. X-ray undetected stars of NGC 2516. Columns report: identifier from Jeffries et al. (2001), $V, B-V, V-I$, exposure time (ks), upper limit to rate (ct/ks), flux and luminosities in the 0.3-7.9 keV band, $\log L_{\mathrm{bol}}$ and the ratio $\log L_{\mathrm{X}} / L_{\mathrm{bol}}$.

\begin{tabular}{|c|c|c|c|c|c|c|c|c|c|}
\hline Opt. Id. & $\begin{array}{l}V \\
\text { mag }\end{array}$ & $\begin{array}{l}B-V \\
\text { mag }\end{array}$ & $\begin{array}{l}V-I \\
\text { mag }\end{array}$ & $\begin{array}{l}\text { Time } \\
\text { ks }\end{array}$ & $\begin{array}{l}\text { Limit rate } \\
\mathrm{ct} \mathrm{ks}^{-1}\end{array}$ & $\begin{array}{l}\text { Lim. } \log f_{\mathrm{X}} \\
\text { erg s}^{-1} \mathrm{~cm}^{-2}\end{array}$ & $\begin{array}{l}\text { Lim. } \log L_{\mathrm{X}} \\
\text { erg s }^{-1}\end{array}$ & $\begin{array}{l}\log L_{\text {bol }} \\
\mathrm{erg} \mathrm{s}^{-1}\end{array}$ & $\log L_{\mathrm{X}} / L_{\mathrm{bol}}$ \\
\hline 3680 & 17.238 & 1.537 & 1.909 & 11.28 & $\leq 1.321$ & $\leq-13.9$ & $\leq 29.35$ & 32.34 & $\leq-2.99$ \\
\hline 3748 & 15.753 & 1.211 & 1.446 & 11.28 & 1.443 & -13.86 & 29.39 & 32.84 & -3.45 \\
\hline 3777 & 20.005 & $\ldots$ & 3.113 & 11.34 & 1.439 & -13.86 & 29.39 & 31.51 & -2.12 \\
\hline 3891 & 17.183 & 1.564 & 2.022 & 11.49 & 1.324 & -13.9 & 29.35 & 32.18 & -2.83 \\
\hline 4069 & 19.661 & $\ldots$ & 2.964 & 11.73 & 1.35 & -13.89 & 29.36 & 31.55 & -2.19 \\
\hline 4072 & 18.981 & $\ldots$ & 2.47 & 12.14 & 1.253 & -13.92 & 29.33 & 31.76 & -2.43 \\
\hline 4146 & 20.693 & $\ldots$ & 3.361 & 27.16 & 1.077 & -13.99 & 29.26 & 31.42 & -2.16 \\
\hline 4187 & 14.492 & 1.112 & 1.214 & 23.01 & 0.87 & -14.08 & 29.17 & 33.08 & -3.91 \\
\hline 4229 & 14.797 & 1.114 & 1.145 & 22.38 & 0.89 & -14.07 & 29.18 & 33.15 & -3.97 \\
\hline 4254 & 19.564 & $\ldots$ & 2.835 & 22.32 & 0.859 & -14.09 & 29.17 & 31.59 & -2.42 \\
\hline 4322 & 19.858 & $\ldots$ & 2.795 & 28.66 & 0.787 & -14.13 & 29.13 & 31.6 & -2.47 \\
\hline 4364 & 19.245 & 1.601 & 2.884 & 14.12 & 1.44 & -13.86 & 29.39 & 31.58 & -2.19 \\
\hline 4397 & 18.385 & 1.646 & 2.357 & 29.49 & 0.842 & -14.1 & 29.16 & 31.86 & -2.7 \\
\hline 4432 & 18.438 & 1.565 & 2.374 & 19.51 & 1.205 & -13.94 & 29.31 & 31.85 & -2.54 \\
\hline 4448 & 14.674 & 1.126 & 1.277 & 33.96 & 0.728 & -14.16 & 29.09 & 33 & -3.91 \\
\hline 4473 & 20.164 & $\ldots$ & 3.155 & 25.8 & 0.858 & -14.09 & 29.16 & 31.49 & -2.33 \\
\hline 4479 & 19.411 & $\ldots$ & 2.572 & 58.82 & 0.582 & -14.26 & 29 & 31.71 & -2.71 \\
\hline 4530 & 20.774 & $\ldots$ & 3.142 & 36.33 & 0.825 & -14.11 & 29.15 & 31.5 & -2.35 \\
\hline 4574 & 13.02 & 0.671 & 0.762 & 14.56 & 1.378 & -13.88 & 29.37 & 34 & -4.63 \\
\hline 4647 & 19.043 & $\ldots$ & 2.529 & 27.35 & 1.063 & -14 & 29.26 & 31.73 & -2.47 \\
\hline 4648 & 20.739 & $\ldots$ & 3.128 & 17.06 & 1.311 & -13.9 & 29.35 & 31.5 & -2.15 \\
\hline 4718 & 19.27 & $\ldots$ & 2.784 & 17.09 & 1.251 & -13.92 & 29.33 & 31.61 & -2.28 \\
\hline 4727 & 19.783 & $\ldots$ & 2.793 & 64.65 & 0.474 & -14.35 & 28.91 & 31.6 & -2.69 \\
\hline 4808 & 19.942 & $\ldots$ & 2.706 & 74.21 & 0.485 & -14.34 & 28.92 & 31.64 & -2.72 \\
\hline 4836 & 18.702 & $\ldots$ & 2.488 & 32.09 & 0.757 & -14.14 & 29.11 & 31.75 & -2.64 \\
\hline 4840 & 10.609 & 0.256 & 0.321 & 28.45 & 0.833 & -14.1 & 29.15 & 34.67 & -5.52 \\
\hline 4842 & 11.243 & 0.397 & 0.478 & 26.09 & 0.872 & -14.08 & 29.17 & 34.45 & -5.28 \\
\hline 4855 & 14.613 & 1.106 & 1.206 & 67.52 & 0.507 & -14.32 & 28.94 & 33.09 & -4.15 \\
\hline 4889 & 11.651 & 0.449 & 0.58 & 31.99 & 0.771 & -14.14 & 29.12 & 34.31 & -5.19 \\
\hline 4914 & 13.541 & 0.768 & 0.853 & 23.79 & 0.7 & -14.18 & 29.08 & 33.81 & -4.73 \\
\hline 4936 & 20.675 & $\ldots$ & 3.086 & 49.89 & 0.527 & -14.3 & 28.95 & 31.52 & -2.57 \\
\hline 5010 & 18.72 & 1.547 & 2.481 & 88.93 & 0.371 & -14.45 & 28.8 & 31.75 & -2.95 \\
\hline 5018 & 18.906 & $\ldots$ & 2.531 & 8.79 & 1.67 & -13.8 & 29.45 & 31.73 & -2.28 \\
\hline 5079 & 20.711 & $\ldots$ & 3.148 & 127.74 & 0.342 & -14.49 & 28.76 & 31.49 & -2.73 \\
\hline 5088 & 19.493 & $\ldots$ & 2.611 & 44.63 & 0.624 & -14.23 & 29.03 & 31.69 & -2.66 \\
\hline 5107 & 12.297 & 0.535 & 0.631 & 8.77 & 1.342 & -13.89 & 29.36 & 34.21 & -4.85 \\
\hline 5144 & 18.934 & 1.577 & 2.726 & 45.38 & 0.759 & -14.14 & 29.11 & 31.63 & -2.52 \\
\hline 5175 & 20.029 & $\ldots$ & 2.839 & 141.43 & 0.301 & -14.54 & 28.71 & 31.59 & -2.88 \\
\hline 5193 & 19.03 & $\ldots$ & 2.647 & 118.63 & 0.338 & -14.49 & 28.76 & 31.67 & -2.91 \\
\hline 5243 & 19.748 & $\ldots$ & 3.005 & 62.38 & 0.569 & -14.27 & 28.99 & 31.54 & -2.55 \\
\hline 5262 & 15.244 & 1.069 & 1.146 & 155.23 & 0.274 & -14.58 & 28.67 & 33.15 & -4.48 \\
\hline 5285 & 19.306 & $\ldots$ & 2.663 & 51.08 & 0.697 & -14.18 & 29.07 & 31.66 & -2.59 \\
\hline 5363 & 20.554 & $\ldots$ & 3.178 & 46.97 & 0.681 & -14.19 & 29.06 & 31.48 & -2.42 \\
\hline 5429 & 20.192 & $\ldots$ & 3.096 & 69.81 & 0.4 & -14.42 & 28.83 & 31.51 & -2.68 \\
\hline 5499 & 15.227 & 1.124 & 1.222 & 54.36 & 0.632 & -14.22 & 29.03 & 33.07 & -4.04 \\
\hline 5509 & 18.963 & 1.729 & 2.534 & 160.54 & 0.316 & -14.52 & 28.73 & 31.73 & -3 \\
\hline 5526 & 16.943 & 1.488 & 1.842 & 151.82 & 0.301 & -14.54 & 28.71 & 32.45 & -3.74 \\
\hline 5528 & 18.837 & 1.689 & 2.54 & 18.55 & 0.866 & -14.08 & 29.17 & 31.72 & -2.55 \\
\hline 5530 & 15.661 & 1.215 & 1.358 & 140.48 & 0.322 & -14.51 & 28.74 & 32.91 & -4.17 \\
\hline 5533 & 19.636 & 1.699 & 2.786 & 82.61 & 0.398 & -14.42 & 28.83 & 31.61 & -2.78 \\
\hline 5536 & 13.126 & 0.697 & 0.818 & 9.24 & 1.355 & -13.89 & 29.36 & 33.89 & -4.53 \\
\hline 5573 & 20.124 & $\ldots$ & 3.156 & 147.94 & 0.284 & -14.57 & 28.68 & 31.49 & -2.81 \\
\hline 5581 & 11.247 & 0.366 & 0.456 & 60.07 & 0.471 & -14.35 & 28.9 & 34.48 & -5.58 \\
\hline 5626 & 11.066 & 0.507 & 0.617 & 132.89 & 0.326 & -14.51 & 28.74 & 34.24 & -5.5 \\
\hline 5631 & 14.999 & 1.065 & 1.201 & 156.81 & 0.272 & -14.59 & 28.67 & 33.09 & -4.42 \\
\hline 5678 & 14.637 & 1.027 & 1.131 & 51.29 & 0.644 & -14.21 & 29.04 & 33.17 & -4.13 \\
\hline 5685 & 21.043 & $\ldots$ & 3.063 & 29.07 & 0.778 & -14.13 & 29.12 & 31.52 & -2.4 \\
\hline
\end{tabular}


Table C.1. continued.

\begin{tabular}{|c|c|c|c|c|c|c|c|c|c|}
\hline Opt. Id. & $\begin{array}{l}V \\
\text { mag }\end{array}$ & $\begin{array}{l}B-V \\
\text { mag }\end{array}$ & $\begin{array}{l}V-I \\
\text { mag }\end{array}$ & $\begin{array}{l}\text { Time } \\
\text { ks }\end{array}$ & $\begin{array}{l}\text { Limit rate } \\
\mathrm{ct} \mathrm{ks}^{-1}\end{array}$ & $\begin{array}{l}\text { Lim. } \log f_{\mathrm{X}} \\
\mathrm{erg} \mathrm{s}^{-1} \mathrm{~cm}^{-2}\end{array}$ & $\begin{array}{l}\text { Lim. } \log L_{\mathrm{X}} \\
\mathrm{erg} \mathrm{s}^{-1}\end{array}$ & $\begin{array}{l}\log L_{\text {bol }} \\
\mathrm{erg} \mathrm{s}^{-1}\end{array}$ & $\log L_{\mathrm{X}} / L_{\text {bol }}$ \\
\hline 5741 & 17.926 & 1.508 & 2.169 & 17.46 & 0.776 & -14.13 & 29.12 & 32 & -2.88 \\
\hline 5785 & 20.01 & $\ldots$ & 2.831 & 116.58 & 0.365 & -14.46 & 28.79 & 31.59 & -2.8 \\
\hline 5843 & 17.878 & 1.456 & 2.16 & 93.23 & 0.4 & -14.42 & 28.83 & 32.01 & -3.18 \\
\hline 5849 & 16.713 & 1.542 & 1.965 & 14.99 & 0.848 & -14.09 & 29.16 & 32.23 & -3.07 \\
\hline 5871 & 18.767 & 1.608 & 2.38 & 27.94 & 0.898 & -14.07 & 29.18 & 31.84 & -2.66 \\
\hline 5875 & 15.428 & 1.141 & 1.232 & 178.22 & 0.685 & -14.19 & 29.07 & 33.06 & -3.99 \\
\hline 5876 & 18.107 & 1.527 & 2.42 & 186.44 & 0.829 & -14.1 & 29.15 & 31.81 & -2.66 \\
\hline 5878 & 18.623 & 1.642 & 2.47 & 46.57 & 1.224 & -13.93 & 29.32 & 31.76 & -2.44 \\
\hline 5882 & 19.11 & $\ldots$ & 2.481 & 174.73 & 1.229 & -13.93 & 29.32 & 31.75 & -2.43 \\
\hline 5904 & 15.043 & 1.086 & 1.158 & 27.8 & 0.94 & -14.05 & 29.2 & 33.14 & -3.94 \\
\hline 5911 & 14.592 & 1.016 & 1.096 & 187.57 & 0.531 & -14.3 & 28.96 & 33.22 & -4.26 \\
\hline 5972 & 18.458 & 1.6 & 2.341 & 42.93 & 0.563 & -14.27 & 28.98 & 31.87 & -2.89 \\
\hline 5991 & 18.463 & 1.618 & 2.327 & 131.48 & 0.344 & -14.49 & 28.77 & 31.88 & -3.11 \\
\hline 6027 & 12.151 & 0.632 & 0.766 & 56.89 & 0.493 & -14.33 & 28.92 & 33.99 & -5.07 \\
\hline 6036 & 12.078 & 0.562 & 0.699 & 54.06 & 0.464 & -14.36 & 28.9 & 34.08 & -5.18 \\
\hline 6044 & 21.234 & $\ldots$ & 3.231 & 94.48 & 0.46 & -14.36 & 28.89 & 31.46 & -2.57 \\
\hline 6049 & 14.701 & 1.014 & 1.094 & 157.18 & 0.305 & -14.54 & 28.72 & 33.22 & -4.5 \\
\hline 6057 & 20.197 & $\ldots$ & 2.927 & 56.04 & 0.61 & -14.24 & 29.02 & 31.57 & -2.55 \\
\hline 6077 & 20.937 & $\ldots$ & 3.17 & 67.71 & 0.513 & -14.31 & 28.94 & 31.49 & -2.55 \\
\hline 6095 & 16.668 & 1.467 & 1.733 & 156.94 & 0.313 & -14.53 & 28.73 & 32.57 & -3.84 \\
\hline 6096 & 12.053 & 0.515 & 0.619 & 156.54 & 0.288 & -14.56 & 28.69 & 34.23 & -5.54 \\
\hline 6112 & 18.809 & 1.563 & 2.522 & 38.29 & 0.563 & -14.27 & 28.98 & 31.73 & -2.75 \\
\hline 6152 & 19.891 & $\ldots$ & 2.987 & 157.77 & 0.315 & -14.52 & 28.73 & 31.55 & -2.82 \\
\hline 6177 & 10.2 & 0.181 & 0.188 & 62.65 & 0.467 & -14.35 & 28.9 & 34.84 & -5.94 \\
\hline 6204 & 17.645 & 1.586 & 2.169 & 159.83 & 0.297 & -14.55 & 28.7 & 32 & -3.3 \\
\hline 6209 & 14.907 & 1.096 & 1.185 & 45.8 & 0.49 & -14.33 & 28.92 & 33.11 & -4.19 \\
\hline 6222 & 14.951 & $\ldots$ & 1.185 & 46.02 & 0.488 & -14.33 & 28.92 & 33.11 & -4.19 \\
\hline 6238 & 19.34 & 1.605 & 2.721 & 202.96 & 0.231 & -14.66 & 28.59 & 31.63 & -3.04 \\
\hline 6264 & 19.866 & $\ldots$ & 2.731 & 189.93 & 0.257 & -14.61 & 28.64 & 31.63 & -2.99 \\
\hline 6289 & 19.175 & $\ldots$ & 2.858 & 107.31 & 0.449 & -14.37 & 28.88 & 31.59 & -2.71 \\
\hline 6297 & 19.032 & 1.703 & 2.463 & 16.37 & 1.068 & -13.99 & 29.26 & 31.77 & -2.51 \\
\hline 6301 & 13.01 & 0.64 & 0.73 & 64.38 & 0.483 & -14.34 & 28.91 & 34.04 & -5.13 \\
\hline 6323 & 19.629 & $\ldots$ & 2.834 & 103.93 & 0.403 & -14.42 & 28.84 & 31.59 & -2.75 \\
\hline 6377 & 11.742 & 0.463 & 0.559 & 149.97 & 1.145 & -13.96 & 29.29 & 34.35 & -5.06 \\
\hline 6402 & 20.505 & $\ldots$ & 2.973 & 204.72 & 0.249 & -14.63 & 28.63 & 31.55 & -2.92 \\
\hline 6445 & 19.745 & $\ldots$ & 2.752 & 170.95 & 0.279 & -14.58 & 28.68 & 31.61 & -2.93 \\
\hline 6446 & 11.37 & 0.386 & 0.474 & 139.18 & 0.361 & -14.47 & 28.79 & 34.46 & -5.67 \\
\hline 6501 & 12.812 & 0.703 & 0.846 & 219.17 & 0.231 & -14.66 & 28.59 & 33.83 & -5.24 \\
\hline 6575 & 9.913 & 0.2 & 0.251 & 211.36 & 1.784 & -13.77 & 29.48 & 34.79 & -5.31 \\
\hline 6594 & 20.29 & $\ldots$ & 2.966 & 178.37 & 0.253 & -14.62 & 28.63 & 31.55 & -2.92 \\
\hline 6691 & 20.334 & $\ldots$ & 2.981 & 75.8 & 0.411 & -14.41 & 28.85 & 31.55 & -2.7 \\
\hline 6705 & 13.968 & 1.022 & 1.108 & 213.44 & 0.215 & -14.69 & 28.56 & 33.19 & -4.63 \\
\hline 6724 & 14.009 & 1.043 & 1.112 & 193.66 & 0.236 & -14.65 & 28.6 & 33.18 & -4.58 \\
\hline 6725 & 18.357 & 1.556 & 2.367 & 57.17 & 0.522 & -14.3 & 28.95 & 31.85 & -2.9 \\
\hline 6771 & 19.202 & $\ldots$ & 2.517 & 230.76 & 0.194 & -14.73 & 28.52 & 31.73 & -3.21 \\
\hline 6796 & 10.26 & 0.194 & 0.223 & 32.95 & 1.273 & -13.92 & 29.34 & 34.81 & -5.47 \\
\hline 6880 & 13.293 & 0.814 & 0.875 & 210.01 & 0.207 & -14.71 & 28.55 & 33.76 & -5.21 \\
\hline 6912 & 10.875 & 0.318 & 0.328 & 206.25 & 0.229 & -14.66 & 28.59 & 34.53 & -5.94 \\
\hline 6940 & 16.898 & 1.593 & 1.924 & 230.33 & 0.198 & -14.73 & 28.53 & 32.32 & -3.79 \\
\hline 6942 & 11.17 & 0.355 & 0.422 & 133.14 & 0.3 & -14.55 & 28.71 & 34.46 & -5.75 \\
\hline 6949 & 11.25 & 0.376 & 0.456 & 211.72 & 0.216 & -14.69 & 28.57 & 34.48 & -5.91 \\
\hline 6963 & 15.28 & 1.14 & 1.234 & 213.08 & 0.246 & -14.63 & 28.62 & 33.05 & -4.43 \\
\hline 6965 & 17.862 & 1.539 & 2.498 & 122.97 & 0.279 & -14.58 & 28.68 & 31.74 & -3.06 \\
\hline 6996 & 18.703 & $\ldots$ & 2.471 & 203.15 & 1.711 & -13.79 & 29.46 & 31.76 & -2.3 \\
\hline 7041 & 10.198 & 0.159 & 0.163 & 216.32 & 0.207 & -14.71 & 28.55 & 34.9 & -6.35 \\
\hline 7113 & 20.25 & $\ldots$ & 3.182 & 48.67 & 0.7 & -14.18 & 29.08 & 31.48 & -2.4 \\
\hline 7154 & 17.875 & 1.626 & 2.425 & 232.26 & 0.246 & -14.63 & 28.62 & 31.8 & -3.18 \\
\hline 7189 & 11.753 & 0.499 & 0.625 & 229.62 & 0.216 & -14.69 & 28.56 & 34.22 & -5.66 \\
\hline 7206 & 10.638 & 0.261 & 0.332 & 262.95 & 0.192 & -14.74 & 28.51 & 34.66 & -6.15 \\
\hline
\end{tabular}


Table C.1. continued.

\begin{tabular}{|c|c|c|c|c|c|c|c|c|c|}
\hline$\overline{\text { Opt. Id. }}$ & $\begin{array}{l}V \\
\text { mag }\end{array}$ & $\begin{array}{l}B-V \\
\text { mag }\end{array}$ & $\begin{array}{l}V-I \\
\text { mag }\end{array}$ & $\begin{array}{l}\text { Time } \\
\text { ks }\end{array}$ & $\begin{array}{l}\text { Limit rate } \\
\mathrm{ct} \mathrm{ks}^{-1}\end{array}$ & $\begin{array}{l}\text { Lim. } \log f_{X} \\
\text { erg s }^{-1} \mathrm{~cm}^{-2}\end{array}$ & $\begin{array}{l}\text { Lim. } \log L_{\mathrm{X}} \\
\text { erg s }^{-1}\end{array}$ & $\begin{array}{l}\log L_{\text {bol }} \\
\mathrm{erg} \mathrm{s}^{-1}\end{array}$ & $\log L_{\mathrm{X}} / L_{\mathrm{bol}}$ \\
\hline 7207 & 14.723 & 1.018 & 1.103 & 65.64 & 0.563 & -14.27 & 28.98 & 33.2 & -4.22 \\
\hline 7259 & 14.371 & 0.934 & 0.992 & 15.19 & 0.856 & -14.09 & 29.16 & 33.4 & -4.24 \\
\hline 7260 & 18.957 & $\ldots$ & 2.736 & 209.99 & 0.721 & -14.16 & 29.09 & 31.62 & -2.53 \\
\hline 7335 & 11.216 & $\ldots$ & 0.451 & 104.5 & 0.316 & -14.52 & 28.73 & 34.48 & -5.75 \\
\hline 7347 & 11.195 & 0.365 & 0.446 & 105.71 & 0.32 & -14.52 & 28.74 & 34.45 & -5.71 \\
\hline 7352 & 16.28 & $\ldots$ & 1.635 & 109.94 & 2.474 & -13.63 & 29.62 & 32.66 & -3.04 \\
\hline 7419 & 18.702 & $\ldots$ & 2.568 & 217.6 & 1.493 & -13.85 & 29.41 & 31.71 & -2.3 \\
\hline 7442 & 16.904 & 1.464 & 1.822 & 44.8 & 0.563 & -14.27 & 28.98 & 32.47 & -3.49 \\
\hline 7443 & 10.91 & 0.318 & 0.366 & 194.14 & 0.86 & -14.09 & 29.17 & 34.53 & -5.36 \\
\hline 7454 & 14.362 & 0.931 & 1.023 & 78.75 & 0.46 & -14.36 & 28.89 & 33.36 & -4.47 \\
\hline 7480 & 17.322 & 1.483 & 2.004 & 15.33 & 0.912 & -14.06 & 29.19 & 32.19 & -3 \\
\hline 7504 & 20.392 & $\ldots$ & 2.879 & 133.84 & 0.415 & -14.4 & 28.85 & 31.58 & -2.73 \\
\hline 7542 & 19.648 & $\ldots$ & 2.692 & 15.69 & 1.013 & -14.02 & 29.24 & 31.65 & -2.41 \\
\hline 7559 & 21.467 & $\ldots$ & 3.276 & 210.15 & 1.325 & -13.9 & 29.35 & 31.45 & -2.1 \\
\hline 7590 & 13.99 & 0.885 & 0.996 & 228.4 & 1.497 & -13.85 & 29.41 & 33.4 & -3.99 \\
\hline 7599 & 20 & $\ldots$ & 2.949 & 44.24 & 0.551 & -14.28 & 28.97 & 31.56 & -2.59 \\
\hline 7613 & 18.543 & 1.707 & 2.349 & 15.1 & 0.942 & -14.05 & 29.21 & 31.87 & -2.66 \\
\hline 7628 & 19.279 & $\ldots$ & 2.658 & 116.43 & 0.253 & -14.62 & 28.63 & 31.67 & -3.04 \\
\hline 7666 & 20.296 & $\ldots$ & 3.115 & 235.26 & 1.515 & -13.84 & 29.41 & 31.51 & -2.1 \\
\hline 7721 & 14.861 & 1.048 & 1.152 & 49.02 & 0.532 & -14.3 & 28.96 & 33.15 & -4.19 \\
\hline 7734 & 19.465 & $\ldots$ & 2.648 & 106.96 & 0.362 & -14.46 & 28.79 & 31.67 & -2.88 \\
\hline 7771 & 15.112 & 1.16 & 1.166 & 142.76 & 0.31 & -14.53 & 28.72 & 33.13 & -4.41 \\
\hline 7788 & 14.832 & 1.11 & 1.269 & 229.83 & 0.199 & -14.72 & 28.53 & 33.01 & -4.48 \\
\hline 7806 & 16.29 & 1.367 & 1.597 & 51.76 & 0.675 & -14.19 & 29.06 & 32.69 & -3.63 \\
\hline 7813 & 14.605 & 0.984 & 1.086 & 23.41 & 0.889 & -14.07 & 29.18 & 33.24 & -4.06 \\
\hline 7838 & 10.415 & 0.379 & 0.47 & 249.04 & 0.181 & -14.76 & 28.49 & 34.46 & -5.97 \\
\hline 7842 & 11.111 & 0.353 & 0.4 & 59.06 & 0.504 & -14.32 & 28.93 & 34.46 & -5.53 \\
\hline 7900 & 11.254 & 0.477 & 0.614 & 61.21 & 0.578 & -14.26 & 28.99 & 34.24 & -5.25 \\
\hline 7937 & 15.359 & 1.171 & 1.265 & 215.48 & 0.26 & -14.61 & 28.65 & 33.01 & -4.36 \\
\hline 7984 & 18.687 & 1.625 & 2.381 & 51.3 & 0.57 & -14.27 & 28.99 & 31.84 & -2.85 \\
\hline 7985 & 17.36 & 1.511 & 2.279 & 19.28 & 0.87 & -14.08 & 29.17 & 31.92 & -2.75 \\
\hline 8031 & 18.989 & $\ldots$ & 2.742 & 196.64 & 0.235 & -14.65 & 28.6 & 31.62 & -3.02 \\
\hline 8047 & 16.116 & 1.356 & 1.606 & 253.51 & 3.347 & -13.5 & 29.76 & 32.68 & -2.92 \\
\hline 8055 & 14.951 & 1.077 & 1.136 & 289.12 & 0.175 & -14.78 & 28.48 & 33.16 & -4.68 \\
\hline 8058 & 10.725 & 0.328 & 0.445 & 297.54 & 0.391 & -14.43 & 28.82 & 34.5 & -5.68 \\
\hline 8132 & 15.717 & 1.201 & 1.324 & 118.71 & 0.388 & -14.43 & 28.82 & 32.94 & -4.12 \\
\hline 8138 & 19.128 & $\ldots$ & 2.508 & 297.25 & 0.527 & -14.3 & 28.95 & 31.74 & -2.79 \\
\hline 8166 & 15.164 & 1.101 & 1.217 & 276.94 & 0.177 & -14.77 & 28.48 & 33.07 & -4.59 \\
\hline 8219 & 19.204 & $\ldots$ & 2.815 & 60.52 & 0.603 & -14.24 & 29.01 & 31.6 & -2.59 \\
\hline 8256 & 13.507 & 0.902 & 0.998 & 59.23 & 2.821 & -13.57 & 29.68 & 33.39 & -3.71 \\
\hline 8262 & 14.215 & 0.95 & 0.982 & 186.6 & 1.693 & -13.79 & 29.46 & 33.44 & -3.98 \\
\hline 8289 & 20.446 & $\ldots$ & 2.979 & 83.79 & 0.435 & -14.38 & 28.87 & 31.55 & -2.68 \\
\hline 8298 & 14.007 & 1.061 & 1.146 & 117.46 & 0.389 & -14.43 & 28.82 & 33.15 & -4.33 \\
\hline 8313 & 10.439 & 0.275 & 0.285 & 166.09 & 0.248 & -14.63 & 28.63 & 34.63 & -6 \\
\hline 8320 & 14.821 & 1.055 & 1.164 & 15.04 & 0.974 & -14.03 & 29.22 & 33.13 & -3.91 \\
\hline 8340 & 10.117 & 0.149 & 0.168 & 295.59 & 0.341 & -14.49 & 28.76 & 34.92 & -6.16 \\
\hline 8359 & 18.162 & 1.57 & 2.217 & 107.84 & 0.405 & -14.42 & 28.84 & 31.96 & -3.12 \\
\hline 8385 & 19.621 & $\ldots$ & 2.866 & 15.08 & 0.974 & -14.03 & 29.22 & 31.58 & -2.36 \\
\hline 8391 & 20.527 & $\ldots$ & 2.912 & 245.82 & 0.189 & -14.75 & 28.51 & 31.57 & -3.06 \\
\hline 8401 & 18.855 & $\ldots$ & 2.578 & 145.76 & 0.323 & -14.51 & 28.74 & 31.71 & -2.97 \\
\hline 8465 & 13.291 & 0.696 & 0.812 & 136.01 & 0.343 & -14.49 & 28.77 & 33.91 & -5.14 \\
\hline 8509 & 18.401 & 1.57 & 2.418 & 122.89 & 0.304 & -14.54 & 28.71 & 31.81 & -3.1 \\
\hline 8524 & 10.972 & 0.334 & 0.398 & 293.83 & 0.163 & -14.81 & 28.44 & 34.49 & -6.05 \\
\hline 8531 & 15.479 & 1.211 & 1.299 & 229.17 & 0.207 & -14.71 & 28.55 & 32.96 & -4.41 \\
\hline 8591 & 18.214 & 1.604 & 2.617 & 237.27 & 0.205 & -14.71 & 28.54 & 31.69 & -3.15 \\
\hline 8678 & 18.24 & 1.474 & 2.338 & 126.88 & 1.385 & -13.88 & 29.37 & 31.88 & -2.51 \\
\hline 8714 & 19.74 & $\ldots$ & 2.679 & 251.88 & 0.186 & -14.75 & 28.5 & 31.65 & -3.15 \\
\hline 8727 & 19.639 & $\ldots$ & 2.713 & 295.11 & 0.161 & -14.82 & 28.44 & 31.64 & -3.2 \\
\hline 8801 & 19.95 & $\ldots$ & 2.766 & 201.11 & 0.222 & -14.68 & 28.58 & 31.61 & -3.03 \\
\hline
\end{tabular}


Table C.1. continued.

\begin{tabular}{|c|c|c|c|c|c|c|c|c|c|}
\hline$\overline{\text { Opt. Id. }}$ & $\begin{array}{l}V \\
\text { mag }\end{array}$ & $\begin{array}{l}B-V \\
\text { mag }\end{array}$ & $\begin{array}{l}V-I \\
\text { mag }\end{array}$ & $\begin{array}{l}\text { Time } \\
\text { ks }\end{array}$ & $\begin{array}{l}\text { Limit rate } \\
\text { ct ks }{ }^{-1}\end{array}$ & $\begin{array}{l}\text { Lim. } \log f_{X} \\
\text { erg s }^{-1} \mathrm{~cm}^{-2}\end{array}$ & $\begin{array}{l}\text { Lim. } \log L_{\mathrm{X}} \\
\mathrm{erg} \mathrm{s}^{-1}\end{array}$ & $\begin{array}{l}\log L_{\mathrm{bol}} \\
\mathrm{erg} \mathrm{s}^{-1}\end{array}$ & $\log L_{\mathrm{X}} / L_{\text {bol }}$ \\
\hline 8827 & 14.35 & 1.001 & 1.059 & 163.56 & 0.267 & -14.6 & 28.66 & 33.29 & -4.63 \\
\hline 8917 & 10.379 & 0.186 & 0.204 & 164.33 & 0.274 & -14.58 & 28.67 & 34.83 & -6.16 \\
\hline 8920 & 11.682 & 0.475 & 0.606 & 236.6 & 0.234 & -14.65 & 28.6 & 34.26 & -5.66 \\
\hline 8923 & 13.974 & 1.031 & 1.099 & 255.74 & 3.802 & -13.44 & 29.81 & 33.21 & -3.4 \\
\hline 8949 & 15.842 & $\ldots$ & 1.539 & 131.64 & 0.397 & -14.42 & 28.83 & 32.75 & -3.92 \\
\hline 8950 & 17.781 & 1.461 & 2.169 & 14.89 & 1.064 & -14 & 29.26 & 32 & -2.74 \\
\hline 9058 & 20.699 & $\ldots$ & 2.972 & 247.2 & 0.232 & -14.66 & 28.6 & 31.55 & -2.95 \\
\hline 9088 & 21.486 & $\ldots$ & 3.348 & 16.75 & 0.899 & -14.07 & 29.19 & 31.42 & -2.23 \\
\hline 9146 & 14.125 & 1.041 & 1.12 & 225.17 & 0.202 & -14.72 & 28.54 & 33.18 & -4.64 \\
\hline 9153 & 19.193 & $\ldots$ & 2.589 & 293.99 & 0.462 & -14.36 & 28.9 & 31.7 & -2.8 \\
\hline 9197 & 13.421 & 0.727 & 0.845 & 16.13 & 0.892 & -14.07 & 29.18 & 33.83 & -4.65 \\
\hline 9214 & 15.281 & 1.146 & 1.255 & 99.3 & 0.335 & -14.5 & 28.76 & 33.03 & -4.27 \\
\hline 9243 & 10.85 & 0.279 & 0.304 & 90.36 & 0.364 & -14.46 & 28.79 & 34.62 & -5.83 \\
\hline 9259 & 19.302 & 1.678 & 2.612 & 159.55 & 0.296 & -14.55 & 28.7 & 31.69 & -2.99 \\
\hline 9270 & 20.123 & $\ldots$ & 2.838 & 169.35 & 0.303 & -14.54 & 28.71 & 31.59 & -2.88 \\
\hline 9361 & 13.59 & 0.779 & 0.889 & 241.27 & 1.967 & -13.73 & 29.52 & 33.73 & -4.21 \\
\hline 9402 & 17.733 & 1.564 & 2.123 & 269.45 & 1.09 & -13.98 & 29.27 & 32.06 & -2.79 \\
\hline 9415 & 18.137 & 1.481 & 2.449 & 259.27 & 1.09 & -13.98 & 29.27 & 31.78 & -2.51 \\
\hline 9427 & 20.279 & $\ldots$ & 3.135 & 70.06 & 0.565 & -14.27 & 28.98 & 31.5 & -2.52 \\
\hline 9459 & 14.932 & 1.026 & 1.147 & 51.49 & 0.556 & -14.28 & 28.98 & 33.15 & -4.17 \\
\hline 9475 & 13.699 & 0.832 & 0.964 & 225.43 & 0.206 & -14.71 & 28.55 & 33.5 & -4.95 \\
\hline 9513 & 14.475 & 0.953 & 1.03 & 198.89 & 0.282 & -14.57 & 28.68 & 33.34 & -4.66 \\
\hline 9590 & 17.851 & 1.551 & 2.167 & 29.83 & 1.121 & -13.97 & 29.28 & 32 & -2.72 \\
\hline 9596 & 15.519 & 1.144 & 1.27 & 247.44 & 0.204 & -14.71 & 28.54 & 33.01 & -4.47 \\
\hline 9600 & 11.714 & 0.41 & 0.59 & 13.61 & 1.186 & -13.95 & 29.31 & 34.29 & -4.98 \\
\hline 9655 & 14.802 & 1.034 & 1.124 & 204.62 & 0.61 & -14.24 & 29.02 & 33.17 & -4.15 \\
\hline 9659 & 11.448 & 0.408 & 0.56 & 245.94 & 0.199 & -14.72 & 28.53 & 34.34 & -5.81 \\
\hline 9682 & 12.308 & 0.551 & 0.676 & 7.88 & 1.79 & -13.77 & 29.48 & 34.11 & -4.63 \\
\hline 9684 & 15.167 & 1.05 & 1.126 & 115.16 & 0.344 & -14.49 & 28.77 & 33.17 & -4.4 \\
\hline 9700 & 19.092 & $\ldots$ & 2.577 & 129.68 & 0.331 & -14.5 & 28.75 & 31.71 & -2.96 \\
\hline 9707 & 15.114 & 1.146 & 1.239 & 37.07 & 0.686 & -14.19 & 29.07 & 33.05 & -3.98 \\
\hline 9791 & 11.231 & 0.381 & 0.459 & 199.94 & 0.256 & -14.61 & 28.64 & 34.47 & -5.83 \\
\hline 9811 & 10.806 & 0.28 & 0.312 & 195.03 & 0.211 & -14.7 & 28.56 & 34.62 & -6.06 \\
\hline 9875 & 15.234 & 1.048 & 1.136 & 270.83 & 0.167 & -14.8 & 28.45 & 33.16 & -4.71 \\
\hline 9878 & 15.461 & 1.17 & 1.237 & 16.83 & 0.809 & -14.11 & 29.14 & 33.05 & -3.91 \\
\hline 9885 & 17.896 & 1.536 & 2.222 & 122.5 & 0.309 & -14.53 & 28.72 & 31.96 & -3.24 \\
\hline 9939 & 19.167 & $\ldots$ & 2.858 & 258.03 & 0.187 & -14.75 & 28.5 & 31.59 & -3.09 \\
\hline 9959 & 17.183 & 1.62 & 2.077 & 219.69 & 0.244 & -14.63 & 28.62 & 32.12 & -3.5 \\
\hline 9961 & 20.698 & $\ldots$ & 3.355 & 264.82 & 0.192 & -14.74 & 28.51 & 31.42 & -2.91 \\
\hline 10077 & 19.089 & $\ldots$ & 2.804 & 228.61 & 7.577 & -13.14 & 30.11 & 31.6 & -1.49 \\
\hline 10129 & 16.482 & 1.422 & 1.653 & 180.47 & 0.242 & -14.64 & 28.62 & 32.65 & -4.03 \\
\hline 10132 & 14.85 & 1.157 & 1.221 & 233.22 & 0.179 & -14.77 & 28.48 & 33.07 & -4.59 \\
\hline 10152 & 13.035 & 0.711 & 0.845 & 173.71 & 0.273 & -14.59 & 28.67 & 33.83 & -5.16 \\
\hline 10162 & 17.617 & 1.584 & 2.042 & 243.92 & 0.192 & -14.74 & 28.52 & 32.15 & -3.63 \\
\hline 10165 & 19.103 & $\ldots$ & 2.562 & 50.17 & 0.623 & -14.23 & 29.03 & 31.71 & -2.68 \\
\hline 10281 & 13.426 & 0.748 & 0.824 & 64.09 & 0.464 & -14.36 & 28.9 & 33.88 & -4.98 \\
\hline 10284 & 15.306 & 1.182 & 1.274 & 53.28 & 0.596 & -14.25 & 29.01 & 33 & -3.99 \\
\hline 10285 & 19.222 & 1.595 & 2.488 & 50.43 & 0.622 & -14.23 & 29.03 & 31.75 & -2.72 \\
\hline 10298 & 20.556 & $\ldots$ & 3.386 & 224.53 & 0.206 & -14.71 & 28.55 & 31.41 & -2.86 \\
\hline 10301 & 11.534 & 0.444 & 0.542 & 167.96 & 0.27 & -14.59 & 28.66 & 34.37 & -5.71 \\
\hline 10365 & 15.874 & 1.255 & 1.467 & 187.65 & 0.846 & -14.09 & 29.16 & 32.82 & -3.66 \\
\hline 10368 & 15.475 & 1.245 & 1.311 & 43.56 & 0.545 & -14.29 & 28.97 & 32.95 & -3.98 \\
\hline 10370 & 18.136 & 1.65 & 2.603 & 51.65 & 0.607 & -14.24 & 29.01 & 31.69 & -2.68 \\
\hline 10383 & 17.736 & 1.512 & 2.152 & 13.09 & 1.29 & -13.91 & 29.34 & 32.02 & -2.68 \\
\hline 10398 & 17.395 & 1.481 & 2.216 & 61.82 & 0.551 & -14.28 & 28.97 & 31.96 & -2.99 \\
\hline 10401 & 18.516 & 1.51 & 2.42 & 59.9 & 0.565 & -14.27 & 28.98 & 31.81 & -2.83 \\
\hline 10496 & 19.516 & $\ldots$ & 2.577 & 13.01 & 1.239 & -13.93 & 29.32 & 31.71 & -2.39 \\
\hline 10503 & 17.023 & 1.502 & 1.879 & 44.1 & 0.542 & -14.29 & 28.96 & 32.39 & -3.43 \\
\hline 10505 & 20.229 & $\ldots$ & 2.829 & 16.01 & 2.257 & -13.67 & 29.58 & 31.59 & -2.01 \\
\hline
\end{tabular}


Table C.1. continued.

\begin{tabular}{|c|c|c|c|c|c|c|c|c|c|}
\hline$\overline{\text { Opt. Id. }}$ & $\begin{array}{l}V \\
\text { mag }\end{array}$ & $\begin{array}{l}B-V \\
\text { mag }\end{array}$ & $\begin{array}{l}V-I \\
\text { mag }\end{array}$ & $\begin{array}{l}\text { Time } \\
\text { ks }\end{array}$ & $\begin{array}{l}\text { Limit rate } \\
\mathrm{ct} \mathrm{ks}^{-1}\end{array}$ & $\begin{array}{l}\text { Lim. } \log f_{X} \\
\text { erg s }^{-1} \mathrm{~cm}^{-2}\end{array}$ & $\begin{array}{l}\text { Lim. } \log L_{\mathrm{X}} \\
\text { erg s }^{-1}\end{array}$ & $\begin{array}{l}\log L_{\text {bol }} \\
\mathrm{erg} \mathrm{s}^{-1}\end{array}$ & $\log L_{\mathrm{X}} / L_{\mathrm{bol}}$ \\
\hline 10527 & 19.077 & 1.623 & 2.594 & 97.44 & 0.348 & -14.48 & 28.77 & 31.7 & -2.93 \\
\hline 10547 & 14.64 & 1.012 & 1.063 & 196.72 & 0.24 & -14.64 & 28.61 & 33.29 & -4.68 \\
\hline 10588 & 20.11 & $\ldots$ & 3.179 & 124.33 & 0.328 & -14.51 & 28.75 & 31.48 & -2.73 \\
\hline 10606 & 19.275 & 1.565 & 2.556 & 42 & 0.754 & -14.14 & 29.11 & 31.72 & -2.61 \\
\hline 10667 & 17.807 & 1.695 & 2.196 & 119.7 & 0.347 & -14.48 & 28.77 & 31.97 & -3.2 \\
\hline 10723 & 13.664 & 0.783 & 0.965 & 213.75 & 0.244 & -14.63 & 28.62 & 33.5 & -4.88 \\
\hline 10735 & 14.166 & 1.09 & 1.196 & 150.9 & 0.279 & -14.58 & 28.68 & 33.1 & -4.42 \\
\hline 10736 & 12.043 & 0.668 & 0.763 & 62.92 & 0.643 & -14.21 & 29.04 & 33.99 & -4.95 \\
\hline 10760 & 20.237 & $\ldots$ & 2.92 & 132.66 & 0.321 & -14.52 & 28.74 & 31.57 & -2.83 \\
\hline 10768 & 18.022 & $\ldots$ & 2.331 & 166.14 & 0.272 & -14.59 & 28.67 & 31.88 & -3.21 \\
\hline 10783 & 13.479 & 0.733 & 0.8 & 38.12 & 0.797 & -14.12 & 29.13 & 33.93 & -4.8 \\
\hline 10796 & 20.088 & $\ldots$ & 2.917 & 34.24 & 0.716 & -14.17 & 29.09 & 31.57 & -2.48 \\
\hline 10811 & 10.884 & 0.271 & 0.323 & 49.13 & 0.628 & -14.22 & 29.03 & 34.64 & -5.61 \\
\hline 10832 & 21.532 & $\ldots$ & 3.552 & 61.96 & 0.665 & -14.2 & 29.05 & 31.34 & -2.29 \\
\hline 10848 & 19.947 & $\ldots$ & 2.811 & 191.5 & 0.261 & -14.6 & 28.65 & 31.6 & -2.95 \\
\hline 10877 & 21.256 & $\ldots$ & 3.36 & 194.03 & 1.988 & -13.72 & 29.53 & 31.42 & -1.89 \\
\hline 11014 & 13.17 & 0.687 & 0.775 & 153.79 & 0.294 & -14.55 & 28.7 & 33.97 & -5.27 \\
\hline 11029 & 14.801 & 1.051 & 1.124 & 195.66 & 0.267 & -14.6 & 28.66 & 33.17 & -4.51 \\
\hline 11031 & 13.892 & 0.822 & 0.999 & 52.01 & 0.656 & -14.21 & 29.05 & 33.39 & -4.34 \\
\hline 11039 & 20.733 & $\ldots$ & 3.021 & 162.83 & 0.881 & -14.08 & 29.18 & 31.54 & -2.36 \\
\hline 11060 & 19.302 & $\ldots$ & 2.629 & 138.66 & 0.311 & -14.53 & 28.72 & 31.68 & -2.96 \\
\hline 11136 & 20.278 & $\ldots$ & 3.026 & 40.42 & 0.651 & -14.21 & 29.04 & 31.54 & -2.5 \\
\hline 11142 & 17.442 & 1.611 & 2.261 & 133.27 & 0.815 & -14.11 & 29.14 & 31.93 & -2.79 \\
\hline 11195 & 19.685 & $\ldots$ & 2.711 & 109.92 & 0.426 & -14.39 & 28.86 & 31.64 & -2.78 \\
\hline 11231 & 20.039 & $\ldots$ & 3.053 & 157.28 & 0.281 & -14.57 & 28.68 & 31.53 & -2.85 \\
\hline 11235 & 18.101 & 1.445 & -9.999 & 54.09 & 0.539 & -14.29 & 28.96 & 32.57 & -3.61 \\
\hline 11287 & 18.38 & $\ldots$ & 2.357 & 121.38 & 2.211 & -13.68 & 29.58 & 31.86 & -2.28 \\
\hline 11303 & 19.243 & $\ldots$ & 2.562 & 48.2 & 0.751 & -14.15 & 29.11 & 31.71 & -2.6 \\
\hline 11304 & 14.282 & 0.928 & 1.007 & 176.17 & 0.299 & -14.55 & 28.71 & 33.38 & -4.67 \\
\hline 11307 & 13.701 & 0.741 & 0.838 & 161.62 & 0.374 & -14.45 & 28.8 & 33.85 & -5.05 \\
\hline 11363 & 17.845 & 1.583 & 2.249 & 15.78 & 0.904 & -14.07 & 29.19 & 31.94 & -2.75 \\
\hline 11405 & 18.158 & 1.716 & 2.35 & 15.29 & 0.906 & -14.06 & 29.19 & 31.87 & -2.68 \\
\hline 11441 & 14.552 & 0.942 & 1.078 & 91.86 & 0.355 & -14.47 & 28.78 & 33.26 & -4.48 \\
\hline 11520 & 20.515 & $\ldots$ & 3.135 & 36.6 & 0.626 & -14.23 & 29.03 & 31.5 & -2.47 \\
\hline 11549 & 15.091 & 1.075 & 1.19 & 160.13 & 0.291 & -14.56 & 28.69 & 33.11 & -4.42 \\
\hline 11611 & 9.619 & 0.223 & 0.257 & 121.16 & 0.325 & -14.51 & 28.74 & 34.74 & -6 \\
\hline 11634 & 17.665 & 1.666 & 2.047 & 44.22 & 0.761 & -14.14 & 29.11 & 32.15 & -3.04 \\
\hline 11716 & 13.756 & 0.801 & 0.896 & 94.12 & 0.463 & -14.36 & 28.9 & 33.71 & -4.81 \\
\hline 11729 & 14.762 & 1.024 & 1.143 & 36.2 & 0.643 & -14.21 & 29.04 & 33.15 & -4.11 \\
\hline 11788 & 14.593 & 0.991 & 1.047 & 135.59 & 0.344 & -14.49 & 28.77 & 33.31 & -4.54 \\
\hline 11813 & 20.824 & $\ldots$ & 3.422 & 118.05 & 0.799 & -14.12 & 29.13 & 31.4 & -2.27 \\
\hline 11844 & 19.155 & $\ldots$ & 2.557 & 110.25 & 0.336 & -14.5 & 28.76 & 31.72 & -2.96 \\
\hline 11852 & 19.377 & $\ldots$ & 2.594 & 99.5 & 0.344 & -14.49 & 28.77 & 31.7 & -2.93 \\
\hline 11857 & 16.964 & 1.513 & 1.888 & 22.53 & 0.907 & -14.06 & 29.19 & 32.38 & -3.19 \\
\hline 11906 & 19.423 & $\ldots$ & 2.651 & 35.24 & 0.694 & -14.18 & 29.07 & 31.67 & -2.6 \\
\hline 11956 & 15.174 & 1.215 & 1.365 & 67.93 & 0.46 & -14.36 & 28.89 & 32.91 & -4.02 \\
\hline 11969 & 17.868 & 1.545 & 2.153 & 19.05 & 1.139 & -13.97 & 29.29 & 32.02 & -2.73 \\
\hline 11998 & 19.64 & $\ldots$ & 2.74 & 26.5 & 1.031 & -14.01 & 29.24 & 31.62 & -2.38 \\
\hline 12109 & 15.308 & 1.101 & 1.206 & 16.4 & 1.318 & -13.9 & 29.35 & 33.09 & -3.74 \\
\hline 12134 & 14.425 & 1.124 & 1.229 & 77.94 & 0.525 & -14.3 & 28.95 & 33.06 & -4.11 \\
\hline 12206 & 19.447 & $\ldots$ & 2.613 & 93.27 & 0.443 & -14.38 & 28.88 & 31.69 & -2.81 \\
\hline 12210 & 19.811 & $\ldots$ & 2.692 & 46.49 & 0.747 & -14.15 & 29.1 & 31.65 & -2.55 \\
\hline 12216 & 12.879 & 0.683 & 0.813 & 16.35 & 1.299 & -13.91 & 29.34 & 33.9 & -4.56 \\
\hline 12235 & 11.732 & 0.461 & 0.64 & 16.85 & 1.237 & -13.93 & 29.32 & 34.19 & -4.87 \\
\hline 12244 & 20.471 & $\ldots$ & 2.873 & 32.44 & 0.665 & -14.2 & 29.05 & 31.58 & -2.53 \\
\hline 12276 & 16.269 & 1.39 & 1.565 & 89.07 & 0.449 & -14.37 & 28.88 & 32.72 & -3.84 \\
\hline 12362 & 14.385 & 1.092 & 1.176 & 65.74 & 0.495 & -14.33 & 28.93 & 33.12 & -4.19 \\
\hline 12365 & 19.293 & 1.726 & 2.624 & 17.02 & 1.155 & -13.96 & 29.29 & 31.68 & -2.39 \\
\hline 12416 & 10.135 & 0.205 & 0.261 & 36.11 & 0.736 & -14.16 & 29.1 & 34.78 & -5.68 \\
\hline
\end{tabular}


Table C.1. continued.

\begin{tabular}{|c|c|c|c|c|c|c|c|c|c|}
\hline$\overline{\text { Opt. Id. }}$ & $\begin{array}{l}V \\
\text { mag }\end{array}$ & $\begin{array}{l}B-V \\
\text { mag }\end{array}$ & $\begin{array}{l}V-I \\
\text { mag }\end{array}$ & $\begin{array}{l}\text { Time } \\
\text { ks }\end{array}$ & $\begin{array}{l}\text { Limit rate } \\
\text { ct ks }{ }^{-1}\end{array}$ & $\begin{array}{l}\text { Lim. } \log f_{X} \\
\text { erg s }^{-1} \mathrm{~cm}^{-2}\end{array}$ & $\begin{array}{l}\text { Lim. } \log L_{\mathrm{X}} \\
\mathrm{erg} \mathrm{s}^{-1}\end{array}$ & $\begin{array}{l}\log L_{\mathrm{bol}} \\
\mathrm{erg} \mathrm{s}^{-1}\end{array}$ & $\log L_{\mathrm{X}} / L_{\text {bol }}$ \\
\hline 12451 & 16.862 & 1.452 & 1.808 & 70.67 & 0.638 & -14.22 & 29.04 & 32.49 & -3.45 \\
\hline 12553 & 16.967 & 1.533 & 1.86 & 79.25 & 0.488 & -14.33 & 28.92 & 32.42 & -3.5 \\
\hline 12565 & 15.489 & 1.217 & 1.295 & 63.34 & 0.53 & -14.3 & 28.96 & 32.97 & -4.01 \\
\hline 12597 & 15.848 & 1.267 & 1.462 & 34.49 & 0.721 & -14.16 & 29.09 & 32.83 & -3.74 \\
\hline 12600 & 16.561 & 1.491 & 2.015 & 49.97 & 0.784 & -14.13 & 29.13 & 32.18 & -3.05 \\
\hline 12613 & 19.888 & $\ldots$ & 2.849 & 34.29 & 0.903 & -14.07 & 29.19 & 31.59 & -2.4 \\
\hline 12628 & 14.507 & $\ldots$ & 1.135 & 64.28 & 2.727 & -13.59 & 29.67 & 33.16 & -3.49 \\
\hline 12709 & 19.534 & $\ldots$ & 2.701 & 62.89 & 0.541 & -14.29 & 28.96 & 31.64 & -2.68 \\
\hline 12711 & 19.631 & $\ldots$ & 2.777 & 46.54 & 0.782 & -14.13 & 29.12 & 31.61 & -2.49 \\
\hline 12754 & 13.564 & 0.871 & 0.939 & 37.97 & 0.803 & -14.12 & 29.14 & 33.58 & -4.44 \\
\hline 12802 & 16.177 & 1.27 & 1.547 & 43.16 & 0.797 & -14.12 & 29.13 & 32.74 & -3.61 \\
\hline 12805 & 19.955 & $\ldots$ & 2.984 & 42.99 & 0.761 & -14.14 & 29.11 & 31.55 & -2.44 \\
\hline 12835 & 17.275 & 1.433 & 1.971 & 36.8 & 0.961 & -14.04 & 29.21 & 32.22 & -3.01 \\
\hline 12868 & 16.668 & 1.492 & 2.134 & 36.35 & 0.841 & -14.1 & 29.16 & 32.05 & -2.89 \\
\hline 12909 & 11.027 & 0.345 & 0.439 & 35.76 & 0.834 & -14.1 & 29.15 & 34.47 & -5.32 \\
\hline 13065 & 17.474 & 1.519 & 2.079 & 21.35 & 0.969 & -14.04 & 29.22 & 32.11 & -2.89 \\
\hline 13079 & 17.825 & 1.468 & 2.249 & 21.15 & 1.008 & -14.02 & 29.23 & 31.94 & -2.71 \\
\hline 13342 & 19.378 & $\ldots$ & 2.64 & 13.4 & 0.938 & -14.05 & 29.2 & 31.68 & -2.48 \\
\hline 13349 & 14.903 & 1.035 & 1.182 & 13.4 & 0.974 & -14.03 & 29.22 & 33.11 & -3.89 \\
\hline 8947 & 17.87 & 1.521 & 2.306 & 244.55 & 2.089 & -13.7 & 29.55 & 31.9 & -2.35 \\
\hline 5877 & 13.852 & 0.891 & 0.995 & 107.32 & 1.034 & -14.01 & 29.25 & 33.4 & -4.15 \\
\hline 5563 & 11.928 & 0.516 & 0.641 & 16.79 & 0.861 & -14.09 & 29.17 & 34.19 & -5.02 \\
\hline 9869 & 18.449 & 1.591 & 2.371 & 53.11 & 0.634 & -14.22 & 29.03 & 31.85 & -2.82 \\
\hline 8328 & 17.898 & 1.531 & 2.22 & 85.5 & 0.437 & -14.38 & 28.87 & 31.96 & -3.09 \\
\hline 11243 & 15.724 & 1.264 & 1.376 & 177.94 & 0.299 & -14.55 & 28.71 & 32.9 & -4.19 \\
\hline 7870 & 20.956 & $\ldots$ & 3.201 & 280.02 & 0.19 & -14.74 & 28.51 & 31.48 & -2.97 \\
\hline 11823 & 20.089 & $\ldots$ & 2.891 & 119.81 & 0.406 & -14.41 & 28.84 & 31.58 & -2.74 \\
\hline 5130 & 14.299 & 0.925 & 1.001 & 78.3 & 0.453 & -14.37 & 28.89 & 33.39 & -4.5 \\
\hline 6683 & 19.031 & $\ldots$ & 2.479 & 103.97 & 0.329 & -14.51 & 28.75 & 31.75 & -3 \\
\hline 5586 & 12.689 & 0.624 & 0.742 & 15.17 & 1.306 & -13.91 & 29.35 & 34.03 & -4.68 \\
\hline 6745 & 18.471 & 1.676 & 2.602 & 45.67 & 0.867 & -14.08 & 29.17 & 31.69 & -2.52 \\
\hline 15497 & 8.78 & 0.09 & $\ldots$ & 126.83 & 4.285 & -13.5 & 29.75 & 35.19 & -5.44 \\
\hline 15498 & 8.06 & -0.02 & $\ldots$ & 31.37 & 0.736 & -14.27 & 28.98 & 36.9 & -7.92 \\
\hline 15502 & 9.05 & 0.08 & $\ldots$ & 230.59 & 1.848 & -13.87 & 29.38 & 35.27 & -5.89 \\
\hline 15514 & 8.93 & 0.04 & $\ldots$ & 11.95 & 1.436 & -13.98 & 29.27 & 36 & -6.73 \\
\hline 15515 & 8.52 & 0.05 & $\ldots$ & 126.35 & 0.342 & -14.6 & 28.65 & 35.53 & -6.88 \\
\hline 15517 & 7.73 & 0.03 & $\ldots$ & 203.47 & 0.222 & -14.79 & 28.46 & 36.21 & -7.75 \\
\hline 15518 & 9.18 & 0.12 & $\ldots$ & 114.32 & 0.297 & -14.66 & 28.59 & 35.03 & -6.44 \\
\hline 15519 & 9.43 & 0.06 & $\ldots$ & 84.6 & 0.364 & -14.58 & 28.68 & 35.45 & -6.77 \\
\hline 15520 & 6.87 & -0.01 & $\ldots$ & 183.27 & 0.287 & -14.68 & 28.57 & 36.75 & -8.18 \\
\hline 15521 & 9.03 & 0.05 & $\ldots$ & 260.87 & 0.192 & -14.85 & 28.4 & 35.53 & -7.13 \\
\hline 15522 & 8.55 & 0.07 & $\ldots$ & 198.06 & 0.721 & -14.28 & 28.97 & 35.34 & -6.37 \\
\hline 15523 & 9.6 & 0.11 & $\ldots$ & 266.02 & 0.409 & -14.53 & 28.73 & 35.09 & -6.36 \\
\hline 15524 & 9.67 & 0.08 & $\ldots$ & 199.24 & 0.572 & -14.38 & 28.87 & 35.27 & -6.4 \\
\hline 15525 & 7.64 & -0.06 & $\ldots$ & 124.95 & 0.28 & -14.69 & 28.56 & $\ldots$ & $\ldots$ \\
\hline 15526 & 6.71 & 1.28 & $\ldots$ & 261.52 & 0.171 & -14.9 & 28.35 & 32.81 & -4.46 \\
\hline 15527 & 7.23 & 0.05 & $\ldots$ & 264.91 & 0.168 & -14.91 & 28.34 & 35.53 & -7.19 \\
\hline 15528 & 8.21 & 0.02 & $\ldots$ & 238.6 & 0.264 & -14.71 & 28.54 & 36.35 & -7.81 \\
\hline 15529 & 8.57 & 0.05 & $\ldots$ & 291.55 & 0.152 & -14.95 & 28.3 & 35.53 & -7.23 \\
\hline 15530 & 8.76 & 0.04 & $\ldots$ & 235.53 & 0.181 & -14.88 & 28.37 & 36 & -7.63 \\
\hline 15531 & 9.65 & 0.11 & $\ldots$ & 97.6 & 0.368 & -14.57 & 28.68 & 35.09 & -6.41 \\
\hline 15533 & 7.85 & 0.03 & $\ldots$ & 240.98 & 0.245 & -14.75 & 28.51 & 36.21 & -7.7 \\
\hline 15534 & 9.56 & 0.16 & $\ldots$ & 180.84 & 0.286 & -14.68 & 28.57 & 34.9 & -6.33 \\
\hline 15535 & 8.38 & 0.04 & $\ldots$ & 69.38 & 0.471 & -14.46 & 28.79 & 36 & -7.21 \\
\hline 15536 & 5.19 & 1.76 & $\ldots$ & 47.5 & 0.56 & -14.39 & 28.86 & 31.54 & -2.68 \\
\hline 15538 & 8.39 & 0 & $\ldots$ & 118.31 & 0.334 & -14.61 & 28.64 & 36.54 & -7.9 \\
\hline 15539 & 9.21 & 0.11 & $\ldots$ & 86.29 & 3.223 & -13.63 & 29.62 & 35.09 & -5.47 \\
\hline 15541 & 9.4 & 0.11 & $\ldots$ & 277.15 & 0.194 & -14.85 & 28.4 & 35.09 & -6.69 \\
\hline 15543 & 9.45 & 0.08 & $\ldots$ & 153.48 & 0.278 & -14.69 & 28.56 & 35.27 & -6.71 \\
\hline
\end{tabular}


I. Pillitteri et al.: Deep X-ray survey of NGC 2516 with XMM-Newton, Online Material p 24

Table C.1. continued.

\begin{tabular}{llllllllll}
\hline \hline Opt. Id. & $\begin{array}{l}V \\
\mathrm{mag}\end{array}$ & $\begin{array}{l}B-V \\
\mathrm{mag}\end{array}$ & $\begin{array}{l}V-I \\
\mathrm{mag}\end{array}$ & $\begin{array}{l}\text { Time } \\
\mathrm{ks}\end{array}$ & $\begin{array}{l}\text { Limit rate } \\
\mathrm{ct} \mathrm{ks}^{-1}\end{array}$ & $\begin{array}{l}\text { Lim. } \log f_{\mathrm{X}} \\
\mathrm{erg} \mathrm{s}^{-1} \mathrm{~cm}^{-2}\end{array}$ & $\begin{array}{l}\text { Lim. } \log L_{\mathrm{X}} \\
\mathrm{erg} \mathrm{s}^{-1}\end{array}$ & $\begin{array}{l}\log L_{\text {bol }} \\
\mathrm{erg} \mathrm{s}^{-1}\end{array}$ & $\begin{array}{l}\log L_{\mathrm{X}} / L_{\text {bol }} \\
\end{array}$ \\
\hline 15544 & 9.48 & 0.07 & $\ldots$ & 260.53 & 0.204 & -14.83 & 28.43 & 35.34 & -6.91 \\
15545 & 9.56 & 0.18 & $\ldots$ & 77.18 & 0.478 & -14.46 & 28.8 & 34.85 & -6.05 \\
\hline
\end{tabular}


I. Pillitteri et al.: Deep X-ray survey of NGC 2516 with XMM-Newton, Online Material p 25

\section{Appendix D: Finding charts of unidentified sources}
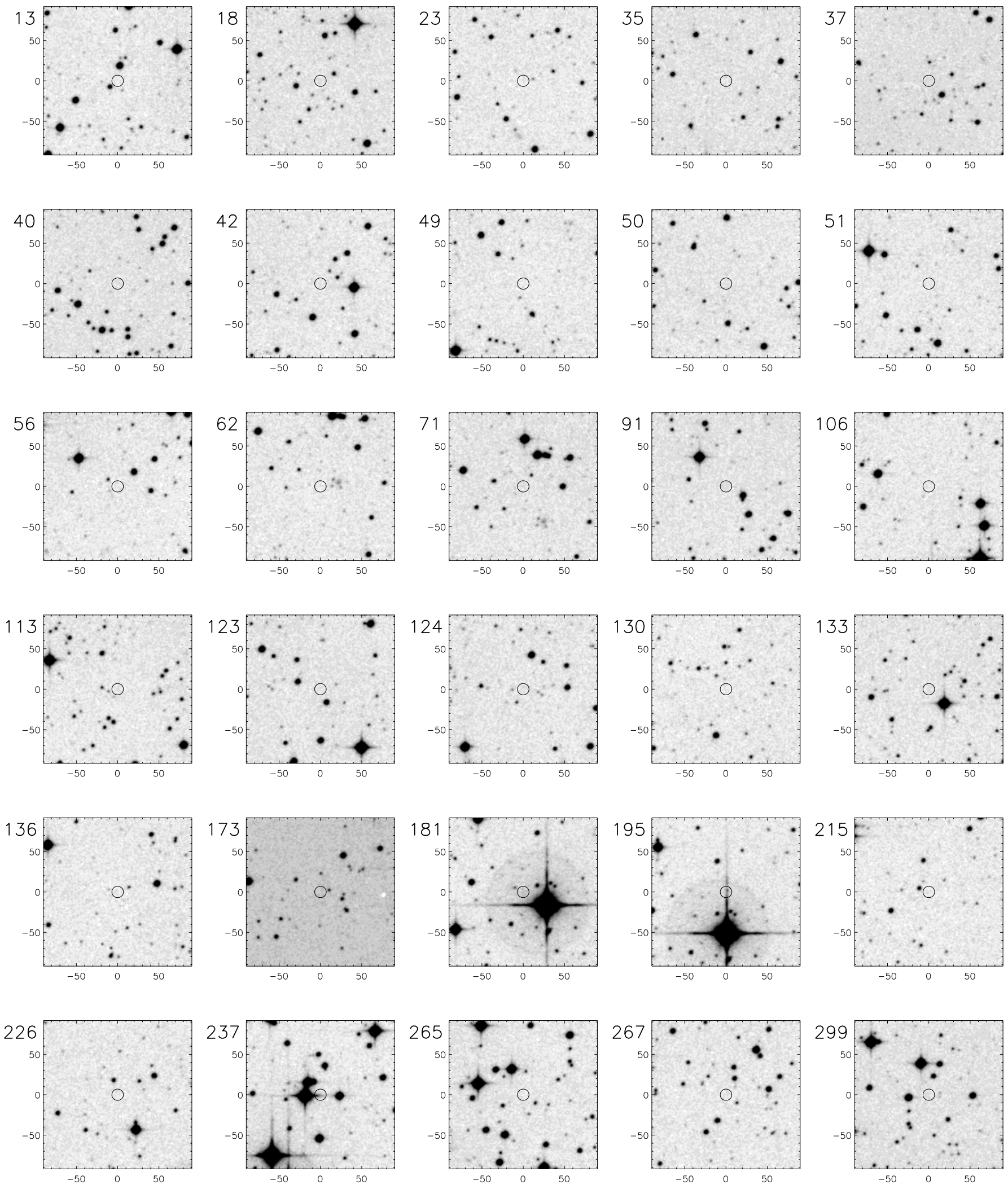

Fig. D.1. DSS IR finding charts $\left(3^{\prime} \times 3^{\prime}\right.$ side $)$ at the position of unidentified X-ray sources. 
I. Pillitteri et al.: Deep X-ray survey of NGC 2516 with XMM-Newton, Online Material p 26
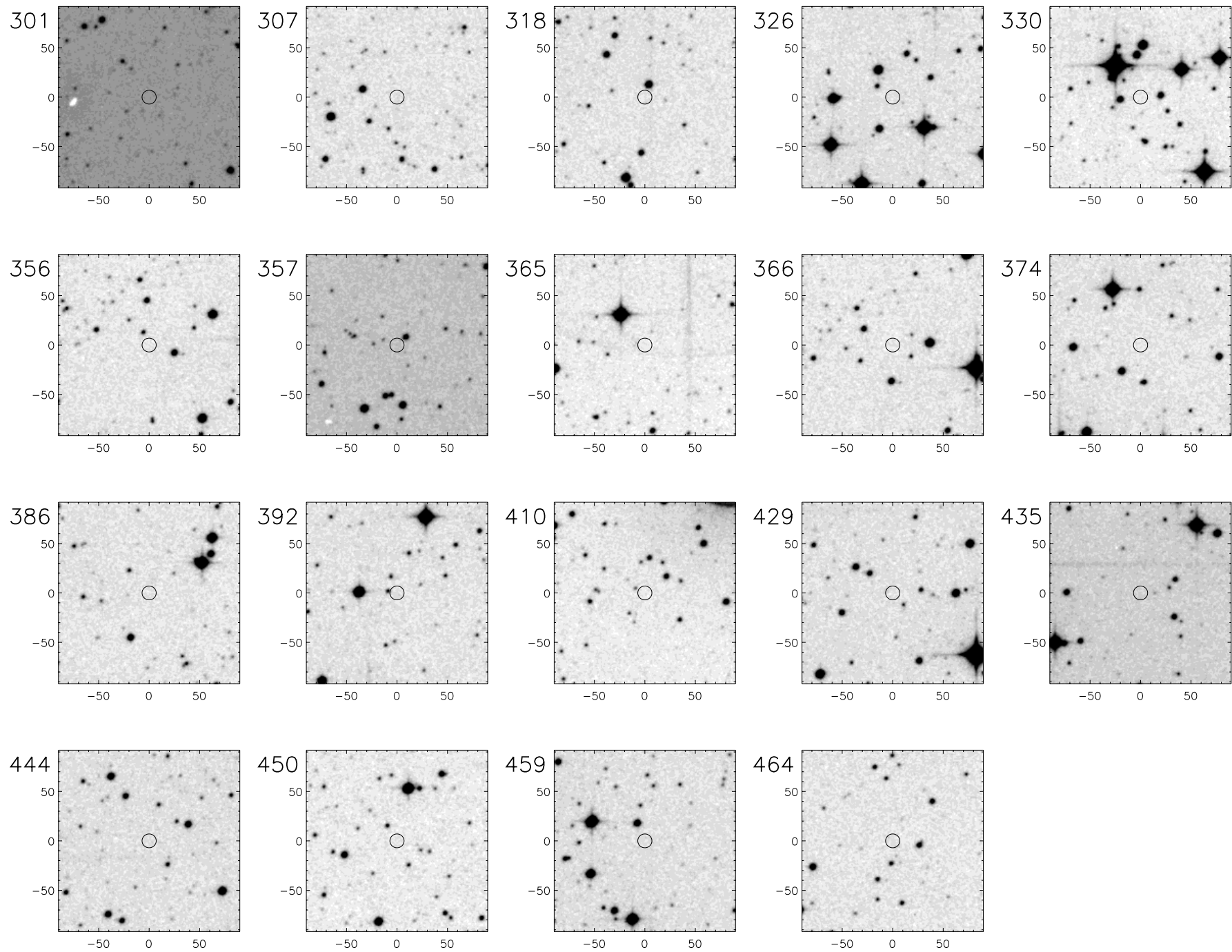

Fig. D.2. As in Fig. D.1. 